THE UNIVERSITY OF MANITOBA

\begin{abstract}
DETERMINATION OF PHASE-LOCKED LOOP STATISTICS
WITH THE AID OF RLC LADDER NETWORKS
\end{abstract}

by

EZZ ISMAIL EL-MASRY

A THESIS

SUBMITTED TO THE FACULTY OF GRADUATE STUDIES

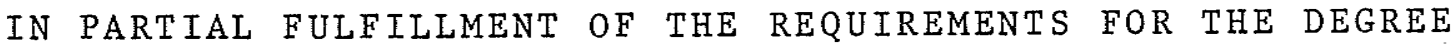
OF DOCTOR OF PHILOSOPHY

DEPARTMENT OF ELECTRICAL ENGINEERING

WINNIPEG, MANITOBA R3T 2N2

october, 1977

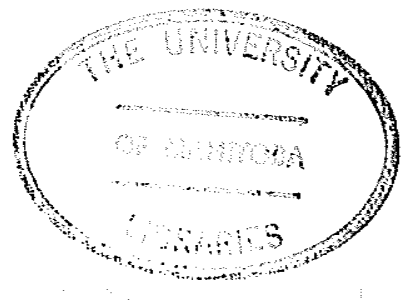




\section{DETERMINATION OF PHASE-LOCKED LOOP STATISTICS \\ WITH THE AID OF RLC LADDER NETWORKS}

BY

\section{EZZ ESMAIL EL-MASRY}

A dissertation submitted to the Faculty of Graduate Studies of the University of Manitoba in partial fullillment of the requirements of the degree of

\section{DOCTOR OF PHILOSOPHY \\ (C) 1977}

Permission has been granted to the L.IBRARY Of THI: UNIVI:RSITY OF: MANITOBA to lend or sel! copies of this dissertation, to the NATIONAL LIBRARY OF CANADA to microtilm this dissertation and to lend or sell copics of the film, and UNIVERSITY MICROFILMS to publish an abstract of this dissertation.

The author reserves other publication rights, and neither the dissertation nor extensive extracts from it may be printed or otherwise reproduced without the author's written permission. 
with love

To my mother

My wife, Iglal

and my son Hisham 
In solving the Fokker-Planck equation as applied to the first-order phase-locked loop, a Fourier series, with time varying coefficients, is used to represent the modulo- $2 \pi$ phase error probability density function. Solutions for the Fourier coefficients are found analytically for some special cases. For these cases the modulo- $2 \pi$ phase error probability density functions are given analytically. However, for the general case, a new technique for computing the Fourier coefficients is developed. The method is based upon the observation that the Fourier coefficients can be interpreted as the state variables in an appropriate RLC ladder network. The Fourier coefficients are computed efficiently by an RLC ladder network simulation on a digital computer. Results are shown for cases involving various signal-to-noise ratios and initial conditions. Also, with the aid of the RLC ladder

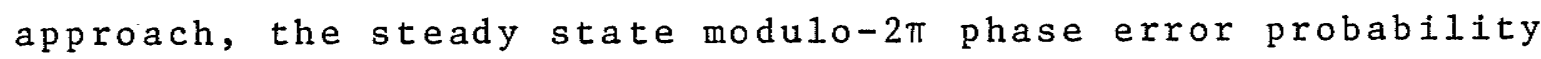
density function and the variance are given in closed forms. This thesis also presents linearization methods applicable to first and second-order phase-locked loops. These have resulted in a systematic method of obtaining the transient statistics of both loops without recourse to the Fokker-P1anck technique. Results for different values of signal-to-noise ratios are shown and compared with those obtained by the RLC ladder approach. 
I wish to express my deep gratitude to Professor G.0. Martens for suggesting the present research area and for providing extremely helpful suggestions while this work was in progress. I remain indebted to him for that and for his continued interest, availability, encouragement, and sincere willingness to advise.

Sincere thanks are given to Professor P.N. Shivakumar for his helpful discussion.

Special thanks go to my wife, Iglal, for her patience, encouragement and inspiration throughout my graduate studies. I also"wish to thank Mrs. Shirley Clubine for typing the thesis. 
ABSTRACT

ACKNOWLEDGEMENT

TABLE OF CONTENTS

LIST OF FIGURES

LIST OF TABLES

(viii)

LIST OF SYMBOLS AND ABBREVIATIONS

I INTRODUCTION

1

1.1 Nature of Phase-Iock 1

1.2 Phase-locked Loop Components and 4 Mechanization

1.3 History and Application 5

II PHASE-LOCKED LOOP ANALYSIS BY USING THE FOKKER-PLANCK TECHNIQUE

2.1 Introduction 9

2.2 Equation Describing the Phase-locked 11 Loop operation

2.3 Fokker-Planck Equation 15

2.3.1 Relations Governing Probability 15 Density Function of Markov Processes

2.3.2 Fokker-Planck Equation for the 18 First-order Phase-1ocked Loop

II MODULO-2 $2 \pi$ PROBABILITY DENSITY FUNCTION OF 21 THE FIRST-ORDER PHASE-LOCKED LOOP

3.1 Introduction 21

3.2 Fourier Series Expansion of the Phase 23 Error Probability Density Function 
3.3 Initial Conditions 26

3.3.1 Deterministic Case 26

3.3.2 Arbitrary Initial Phase 29 Distribution.

3.4 Computation of the Fourier Series 30 Coefficients

3.4.1 Analytic Solutions for the 31 Fourier Coefficients (Special (ases)

$$
\begin{array}{llll}
3.4 .1 \mathrm{a} & \text { Case of } & \beta=0 \text { and } \gamma=0 & 31 \\
3.4 .1 \mathrm{~b} & \text { Case of } & \beta=0 \text { and } \gamma \neq 0 & 36
\end{array}
$$

3.4.2 Approximate solution in Closed 39 Form

3.5 RLC Ladder Network Representation 41

3.5.1 Realization of the RLC Ladder 42 Networks

3.5.2 Stability Study 46

3.5.2a Stability of the Finite 46 RLC Ladder Network

$3.5 .2 b$ Stability of the, 49 Infinite Ladder

3.6 Steady State Analysis 52

IV TRANSIENT STATISTICS OF THE PLL WITHOUT RE- 58 COURSE TO THE FOKKER-PLANCK TECHNIQUE

4.1 Introduction 58

4.2 First-order Phase-locked Loop 58

4.3 Second-order Phase-1ocked Loop 62

V NUMERICAL RESULTS 66

5.1 Truncated Ladder Technique 66

5.2 Linearization Methods 68 
VI

CONCLUSION 90

REFERENCES 92

$\begin{array}{ll}\text { APPENDIX A } & 97\end{array}$

Determination of the Modal Matrix

$Q$ in Equation ( 3.40$)$

APPENDIX B

Evaluating the Constant $\left(d_{n}\right)$ of

Equation (3.42)

APPENDIX C

100

Solution of the System of Equations (3.83)

APPENDIX D

Derivation of Equation (3.49)

APPENDIX E

Solution of the Equations $(3.56)$ and $(3.57)$

APPENDIX F

Derivations of Equations $(3.64),(3.65)$ and $(3.66)$

APPENDIX G

Derivations of Equations (4.25)-(4.29)

APPENDIX H

Derivation of the Fokker-Planck Equation 
Figure 1 Fundamenta1 Hardware Structure of the 2 Phase-1ocked Loop

Figure 2 Phase-locked Loop Model Involving IF 2 Filtering

Figure 3 Phase-locked Loop Modeled Without Noise 14

Figure 4 Phase-1ocked Loop Modeled with Additive 14 Noise

Figure 5 Continuous Markov Process 14

Figure 6 Dirac Delta Function Representation 27

Figure 7 RLC Ladders with Coupling Represented 43 by Voltage and Current Sources

(a) RLC Ladder with State Variables a $(\tau)$

(b) RLC Ladder with State Variables $b_{n}^{n}(\tau)$

Figure 8 RLC Ladder with Coupling Represented $\quad 45$ by Idea 1 Gyrators

Figure 9 RLC Ladders for Stability Study

$$
\begin{aligned}
& \text { (a) RLC Ladder with } R_{n}\left(\text { or } G_{n}\right)=2 n \beta \\
& \text { (b) RLC Ladder with } R_{n}\left(\text { or } G_{n}\right)=2 \beta
\end{aligned}
$$

Figure 10 Configuration at Steady State

(a) Network with Branch Currents and Shunt Voltages Represented by $a_{n}$

(b) Network with Branch Currents and Shunt Voltages Represented by $b_{n}$

Figure 11 Configuration at steady state with $\gamma=0 \quad 54$

Figure 12 Modulo-2 2 Phase Error Probability Density 72

Function $\alpha=0.1, \Phi_{0}=0, \gamma=0$

Figure 13 Modulo-2 2 Phase Error Probability Density 73

Function $\alpha=1.0, \Phi_{0}=0, \gamma=0$

Figure 14 Modulo-2 $\pi$ Phase Error Probability Density 74 Function $\alpha=1.0, \Phi_{0}=0, \gamma=0$

Figure 15 Modulo-2 $\pi$ Phase Error Probability Density 75 Function $\alpha=5.0, \Phi_{0}=0, \alpha=0$ 
Figure 16 Modulo-2 $2 \pi$ Phase Error Probability Density

Function $\alpha=1.0, \Phi_{0}=0, \quad \gamma=\sin \frac{\pi}{4}$

Figure 17 Modulo-2 $2 \pi$ Phase Error Probability Density

Function $\alpha=2.0, \quad \Phi_{0}=\frac{\pi}{2}, \quad \gamma=0$

Eigure 18 Modulo-2 $2 \pi$ Phase Error Variance

Figure 19 Modulo-2 2 Phase Error Probability Density

Function $\alpha=1.0, \quad \gamma=0, \quad P(\Phi, 0)=\frac{1}{2 \pi}$

Figure 20 Modulo-2 2 Phase Error Probability Density

Function $\alpha=1.0, \quad \gamma=\sin \frac{\pi}{4}, P(\Phi, 0)=\frac{i}{2 \pi}$

Figure 21 Modulo-2 $2 \pi$ Phase Error Probability Density

Function $\alpha=5.0, \quad \gamma=0, P(\Phi, 0)=\frac{1}{2 \pi}$

Figure 22 Modulo-2 2 Phase Error Variance, $P(\Phi, 0)=\frac{1}{2 \pi}$

Figure 23 Modulo-2 2 Phase Error Probability Density Funciion $\alpha=0.1, \Phi_{0}=0, \quad \gamma=0$,
$\tau=0.06$.

Figure 24 Modulo-2 2 Phase Error Probability Density Function $\alpha=0.1, \Phi_{0}=0, \gamma^{2}$,
$\tau=0.15$.

Figure 25 Modulo- $2 \pi$ Phase Error Probability Density

Function $\alpha=1.0, \Phi_{0}=0, \quad \gamma=0$,
$\tau=0.06$.

Figure 26 Modulo-2 2 Phase Error Probability Density

Function $\alpha=1.0, \Phi_{0}=0, \gamma=0$, $\tau=0.51$.

Figure 27 Modulo-2 $2 \pi$ Phase Error Probability Density Function $\alpha=0.1, \Phi_{0}=0, \gamma=0$,
$\tau=0.06$.

Figure 28 Modulo-2 $2 \pi$ Phase Error Probability Density Function $\alpha=0.1, \Phi_{0}=0, y=0$,
$\tau=0.15$.

Figure 29 Modulo- $2 \pi$ Phase Error Probability Density Function $\alpha=1.0, \Phi_{0}=0$,
$\tau=0.51$.

Figure 30 The truncated RLC ladder network. 


\section{LIST OF TABLES}

Page

Table I

Various studied conditions for

$P(\Phi, 0)=\delta(\Phi-\Phi)$

70

Table II Various studied conditons for

$P(\Phi, 0)=\frac{1}{2 \pi}$

71 


\section{SYMBOLS AND ABBREVIATIONS}

A

$a_{n}(\tau), b_{n}(r)$ Fourier series coefficients

${ }^{\mathrm{B}} \mathrm{L}$

$\mathrm{C}_{\mathrm{n}}$

$\mathrm{E}$

$e_{0}(t)$

FM

$G_{n}$

$\mathrm{g}_{\mathrm{n}}, \mathrm{r}_{\mathrm{n}}$

$\mathrm{H}(\mathrm{s})$

$h(t)$

IF

$\mathrm{K}$

K

$\mathrm{K}_{2}$

$\mathrm{L}_{\mathrm{n}}$

$\mathrm{N}$

$\frac{0}{2}$

$\mathrm{n}(\mathrm{t})$

$n^{\prime}(t)$

PD

PM

PDE

PLL

$P(\Phi, \tau)$

Frequency modulation
Loop-noise bandwidth

The nth shunt capacitor of the RLC ladder

Input source to the RLC ladder

Loop-filter zero input response

The $\mathrm{n}^{\text {th }}$ shunt conductor of the RLC ladder

Coupling coefficients

Transfer function of the loop filter

Impulse response of the loop filter

Intermediate frequency

The Ioop gain íntor

Voltage-controlled oscillator output rootmean-square amplitude

Voltage-controlled oscillator gain factor

The $n$th series inductor of the RLC 1 adder

Two-sided noise specral density

Additive white gaussian noise

White gausian random process

Phase detector

Phase modulation

Probability density function

Phase-1ocked loop

Modulo-2 $2 \pi$ phase exror PDF 


\begin{tabular}{|c|c|}
\hline$P(\Phi)$ & 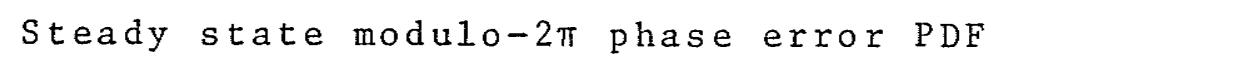 \\
\hline$p(\phi, \tau)$ & Phase error PDF \\
\hline Q & Modal matrix \\
\hline $\mathrm{R}_{\mathrm{n}}$ & The $\mathrm{n}^{\mathrm{th}}$ series resistor of the RLC ladder \\
\hline SNR & Signa1-to-noise ratio \\
\hline $\mathrm{VCO}$ & Voltage-controlled oscillator \\
\hline$\alpha$ & The signal-to-noise ratio in the $100 \mathrm{p}$ bandwidth \\
\hline$\beta$ & $\beta=\frac{1}{\alpha}$ \\
\hline$\gamma$ & Detuned factor \\
\hline$\theta$ & Input phase \\
\hline$\theta^{\prime}$ & vCo output phase \\
\hline$\theta_{1}$ & Input phase relative to the vCO center frequency \\
\hline$\theta_{2}$ & $\begin{array}{l}\text { VCo output phase relative to the VCo center } \\
\text { frequency }\end{array}$ \\
\hline$\Phi$ & Modu1o-2 $\pi$ phase error \\
\hline$\phi$ & Phase error \\
\hline$\sigma_{\phi}^{2}(\tau)$ & Phase error variance \\
\hline$\sigma_{\Phi}^{2}(\tau)$ & Modu $10-2 \pi$ phase error variance \\
\hline$\lambda_{\mathrm{n}}$ & The nth eigen value \\
\hline$\omega$ & Input signal frequency \\
\hline$\omega_{0}$ & Quiescent frequency of the VCo \\
\hline$\tau$ & Normalized time \\
\hline
\end{tabular}


CHAPTER I

INTRODUCTION

Successful transmission of information through a phasecoherent communication system requires, by definition, a receiver capable of determining or estimating the phase and frequency of the received signal with as little error as possible.

In this thesis, we study the noise analysis of a nonlinear device called the phase-locked loop that can be used for tracking the phase of the carrier component of the received signal; this device thus generates a signal suitable for synchronous demodulation. Furthermore, this device can be used for demodulation of angle modulated signals (PM and FM) in the presence of strong noise.

\subsection{Nature of Phase-10ck}

A phase-locked loop (PLL) is a nonlinear device which automatically controls an oscillator or a periodic function generator so as to operate at a constant phase angle relative to a reference signal source. Fig. 1 shows the fundamental hardware structure of the loop with the appropriate signals. The three basic components of a phase-locked loop are:

a) a phase detector (PD),

b) a linear time invariant low pass filter,

and c) a voltage-controlled oscillator (VCO).

The PLL operation can be described as follows:

The phase detector (multiplier) compares the phase of 
$\sqrt{2} A \sin \theta(9)$

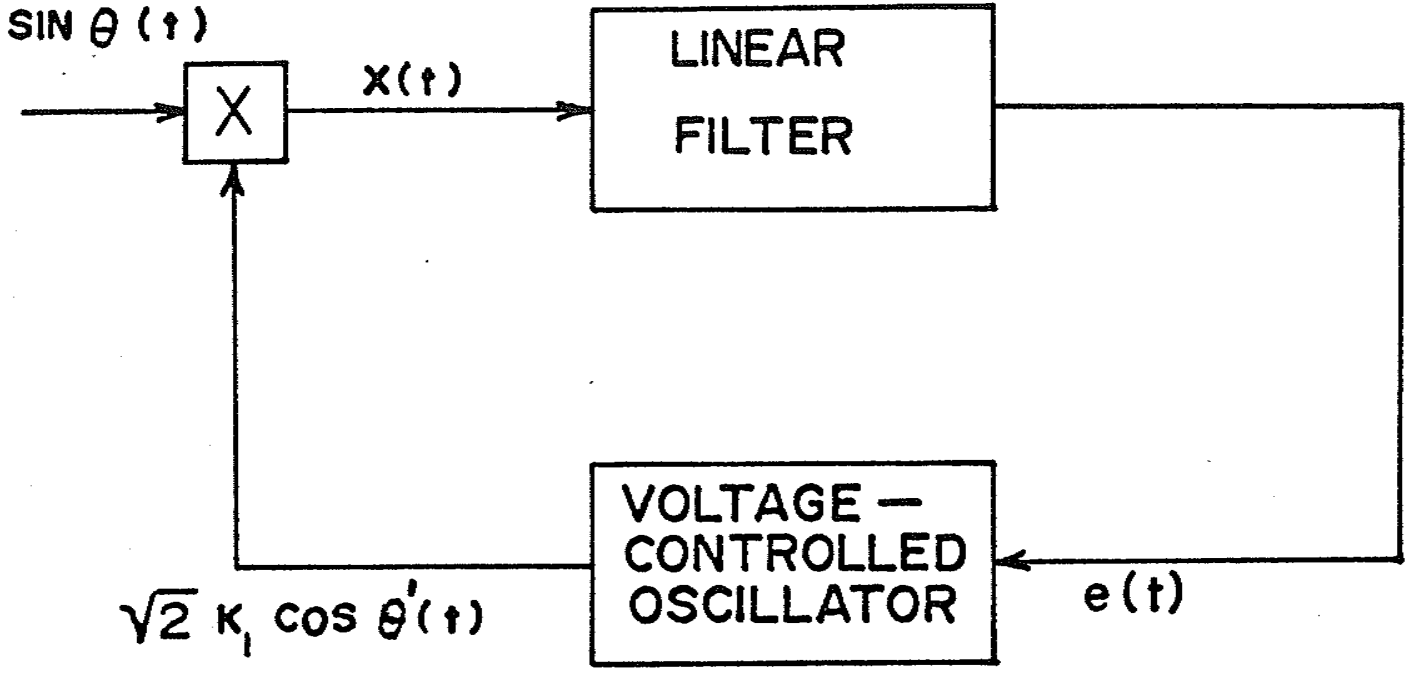

Figure 1. Fundamental Hardware Structure of the Phase-Locked Loop

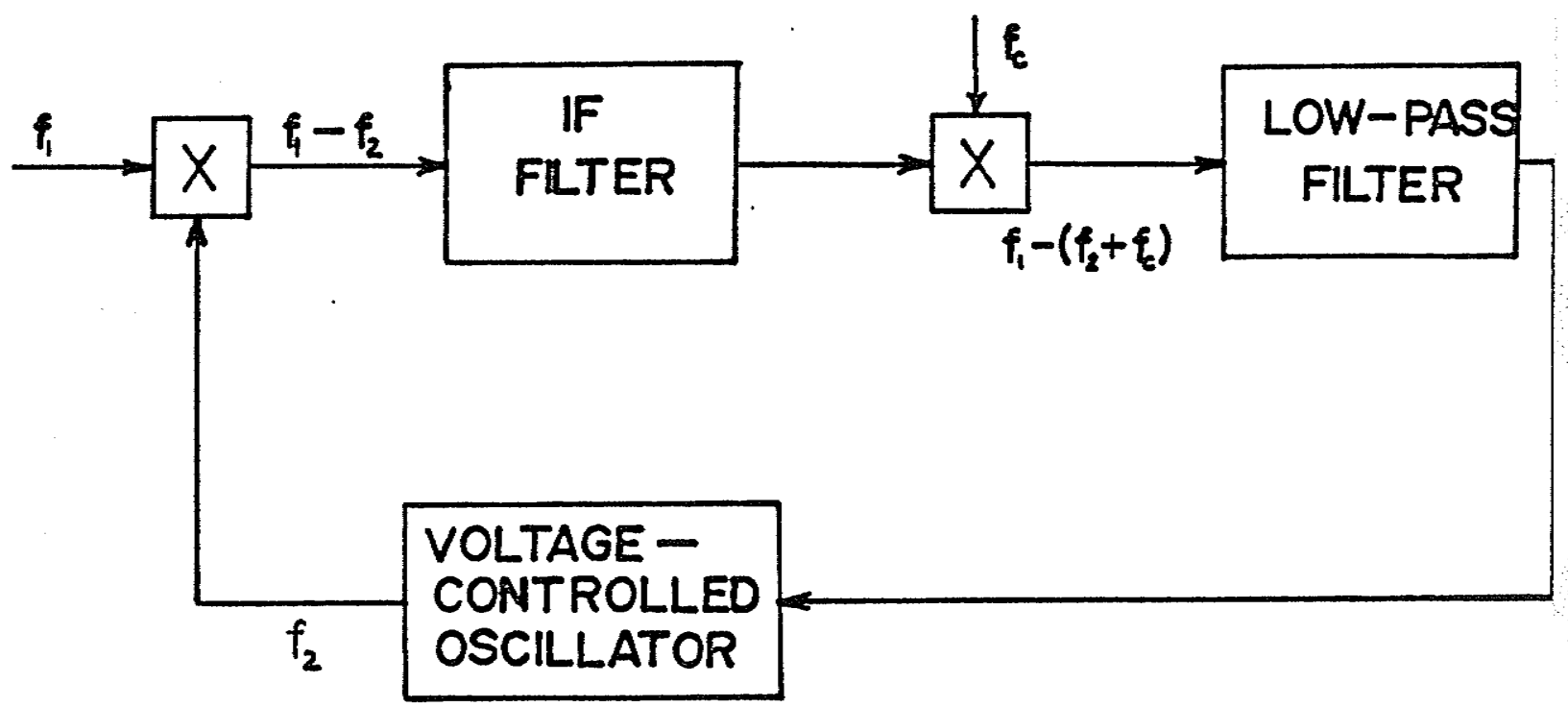

Figure 2. Phase-Locked Loop Model Involving IF Filtering 
a periodic input signal against the phase of the VCo; the output of the PD is a measure of the phase difference between its two inputs. The difference voltage is then filtered by the loop filter and applied to the VCo. The control voltage on the VCo changes the frequency in a direction that reduces the phase difference between the input signal and the local oscillator.

When the loop is "locked", the control voltage is such that the frequency of the VCo is exactly equal to the average frequency of the input signal.

A slightly different explanation may provide a better understanding of the loop operation. Suppose that the incoming signal carries information on its phase or frequency, this signal is inevitably corrupted by additive noise. The task of a phaselock receiver is to reproduce the original signal while removing as much of the noise as possible.

To reproduce the signal the receiver makes use of a local oscillator whose frequency is very close to that expected in the signal. The local oscillator and the incoming signal waveforms are compared with one another by a phase detector whose error output indicates the instantaneous phase difference. To suppress noise, the error is averaged over some length of time and the averaged error is then used to control the frequency of the oscillator.

Two important characteristics of the filter are that the bandwidth should be very small and the filter should automatically track the signal frequency. These features, auto- 
matic tracking and narrow bandwidth, account for the major uses of phaselock receiver. The narrow bandwidth makes it capable of rejecting large amounts of noise.

\subsection{Phase-locked Loop Components and Mechanization}

It is not our purpose in this work to engage in extensive descriptions of the components and equipment which compose the phase-locked 10op. However, since our treatment is necessarily motivated by practical considerations it is necessary to mention the characteristics of components which pertain to their performance.

The VCo is essentially a frequency modulator, of which there are numerous varieties depending on the frequency range of interest. The only essential characteristic required of a phase-1ocked loop VCo is that its frequency displacement from the quiescent frequency be strictly a linear function of the input voltage over the desired frequency range.

The loop filter, as it is considered here, consists of 1 umped passive elements and amplifiers connected to form a low-pass filter. However, in practical receivers some filtering is often performed at an intermediate frequency (IF) by modeling the loop as shown in Fig. 2, particularly when the VCo quiescent frequency is far removed from the receivedsignal frequency. This loop can be reduced to the model of Fig. $1[3]$.

The most complicated device to realize in the phaselocked loop is the multiplier (Phase-detector) because one 
requires a perfect electronic multiplier which does not saturate over the amplitude and frequency range of interest. However, it is shown [3] that essentially identical performance is achieved using practical "chopper" multipliers.

\subsection{History and Application}

One example of phaselock - of a sort - has been in existence for many years. Electric utilities maintain the average frequency of their generators extremely close to 60 $\mathrm{Hz}$, largely so that electric clocks will not gain or lose time. Frequency is regulated by a kind of phaselock loop in which the signal is the time of day, ultimately derived from the Bureau of Standards. This reference time is compared against the time indicated by a clock driven by the utility's generator. The comparing device is a phase detector in fact, a1though not in name, and the turbine and generator constitute a VCo. Any phase (time) discrepancy information is used to adjust the speed of the turbine in a direction that will reduce the discrepancy. Filtering comes in part from inertia of the rotating machinery and electrical inertia of the system 1oad.

This example, in which phase comparison and frequency adjustment are performed on an intermittent basis and with disturbances coming from variations of the load rather than noise on the input signal, is perhaps somewhat strained and atypical. Nonetheless, if one were so inclined, the process could be analyzed on a phaselock basis. 
The first widespread use of phase-locked loops was in the synchronization circuits for color television [2,3].

The application of phaselock technique in space began with the launching of the first American artificial satellites. These vehicles carried low-power ( $10 \mathrm{~mW}) \mathrm{CW}$ transmitters; received signals were correspondingly weak. Due to Doppler shift and the drift of the transmitting oscillator, there was considerable uncertainty about the exact frequency of the received signal. At the 108-MHz frequency originally used the Doppler shift could range over a $\pm 3-\mathrm{KHz}$ interval. With an ordinary, fixed-tuned receiver, bandwidth would therefore have to be at least $6-\mathrm{KHz}$, if not more. However, the signal itself occupies a very narrow spectrum, and could be contained in something like a $6-\mathrm{Hz}$ bandwidth.

Noise power in a receiver is directly proportional to bandwidth. Therefore, if conventional techniques were used, a noise penalty of $30 \mathrm{db}$ would have to be accepted. (The numbers have become even more spectacular as technology has progressed; transmission frequencies have moved up to $\mathrm{S}-\mathrm{band}$, making the Doppler range some $\pm 75 \mathrm{KHz}$, whereas receiver bandwidth as small as $3 \mathrm{~Hz}$ have been achieved. The penalty for conventional techniques would thus be about $47 \mathrm{db}$ ). Such penalties are intolerable and that is why narrow-band, phaselocked, tracking receivers are used.

Noise can be rejected by a narrow-band filter, but if the filter is fixed the signal will almost never fall in the passband and thus for a narrow filter to be usable it must be 
capable of tracking the signal. A phase-locked loop is capable of providing both the narrow bandwidth and the tracking that are needed.

For a Doppler signal the information needed to determine vehicle velocity is the Doppler frequency shift. A phase-lock receiver is well adapted to Doppler recovery, for it has no frequency error when locked.

There are many other applications of the PLL; we refer to $[3]-[10]$.

Having seen that PLL's are used widely in many applications in modern communications dealing with noise, the noise analysis of the PLL is therefore very important.

The main objective of this thesis is the noise analysis of the phase-1ocked loop. This is carried out with the aid of the RLC ladder network approach. This approach facilitates the solution of the stated problem. The ladder network representation of the problem is new, and to the best knowledge of the author, it has not been previously treated in the literature.

The thesis consists of six chapters, including the introduction. In chapter II, derivations of the equation describing the loop operation and the corresponding FokkerPlanck equation are presented. It is also shown how the Fokker-Planck technique can be used to obtain the phase error probability density function ( $P D F$ ) of the first-order PLL. Modu1o-2 $2 \pi$ phase error probability density function of the first-order PLL is dealt with in Chapter III. In this 
chapter the Fourier series expansion of the PDF is presented as we11 as procedures for computing the Fourier coefficients. Analytic solutions for two special cases (namely, Case 1: $\beta=0, \gamma=0$ and Case 2: $\beta=0, \gamma \neq 0)$ are given. An approximate closed form solution, based on the successive approximation method, is also given. The RLC ladder network approach which is used to investigate the stability of the system and to solve for the Fourier coefficients, is introduced. Steady state analysis is also given.

Chapter IV deals with the statistics of the linearized first and second order PLL's without recourse to the FokkerP1anck equation.

The results of the numerical solution are given, and compared with other published results, in Chapter V. Also in this chapter the results of the linearized loop are plotted.

Chapter VI then outlines some concluding remarks. Final1y, nine appendices set out the mathematical proof of some of the confronted equations. 
CHAPTER II

PLL ANALYSIS BY USING THE FOKKER-PLANCK TECHNIQUE

\section{$2.1 \quad$ Introduction}

The applications of the PLI mentioned in Chapter I and many others spurred great interest in the development of phaselocking techniques. Due to the inherent nonlinearity of the loop phase detector, early attempts to analyze phase-1ocked loop behavior involved the use of graphical phase plane methods which are summarized by Viterbi [3]. The initial use of Fokker-Planck technique, which is described in the third part of this chapter, to determine the steady state probability distribution of the first-order loop phase error was accomplished by Tikhonov [11,12]. Since then much work has been done using the Fokker-Planck method of analysis but little has been published dealing with the transient behavior of the phase-error process.

Various techniques have been employed in attempting to statistically describe the phase-error process of first order phase-locked 1oops. Stationary phase-error distributions are well documented by Viterbi [3] and Tikhonov [11]and[12]. Transient solutions of the Fokker-Planck equation have been obtained by Dominiak and Pickholtz [13], Grandoni and Mengali [15] and by ohlson and Rutherford [14]. Dominiak and Pickholtz [13] arrived at a direct numerical solution to the Fokker-Planck equation by subjecting it to the same numerical procedures as applied to the one-dimensional heat-flow equation by Von Neumann 
and Richtmyer [16]. Grandoni and Mengali [15] have proposed an approximate method for solving the Fokker-Planck equation for the first-order PLL. The method consists of 1 mposing a gaussian structure on the probability density function. They have obtained a set of ordinary, simultaneous differential equations for the mean value and the variance of the phase error distribution. These equations have been solved numerically. OhIson and Rutherford [14] have solved the FokkerPlanck equation, as applied to the first-order PLL, numerically. They expressed the solution in terms of the product of two functions, one of which is independent of time. The other function is evaluated numerically on a digital computer for discrete values of time.

This study was started in the belief that the various work in the analysis of the transient behavior of the PLL could be expanded and improved. Dominiak and Pickholtz [13] create serious doubt as to the completeness and validity of their technique in that their conclusion of insignificant build up of probability mass at $\phi= \pm 2 k \pi$ conflicts greatly with qualitative estimates of Viterbi [3] and [17] and Lindsey [10], and with the numerical results obtained by ohlson and Rutherford [14]. Grandoni and Mengali's results [15] do not agree with the exact probability density function, which cannot be gausstan due to the loop nonlinearity, in particular for small signal-to-noise ratio. Ohlson and Rutherford's [14] computation technique is extremely time consuming on any computer. In their approach up to 40,000 1terations [14] and [41] are required for each case to obtain the given results. 


\subsection{Equation Describing the PLL operation}

The stochastic differential equation of the phase error is obtained using the configuration of Fig. 1. The phase detector output is

$$
\begin{aligned}
X(t) & =2 A K_{1} \sin \theta(t) \cos \theta^{\prime}(t) \\
& =A_{1}\left[\sin \left(\theta(t)-\theta^{\prime}(t)\right)+\sin \left(\theta(t)+\theta^{\prime}(t)\right)\right]
\end{aligned}
$$

where $\theta(t)$ and $\theta^{\prime}(t)$ are the input phase and the VCo output phase respectively.

The linear time-invarient, low-pass filter filters out the sum frequency component of its input, $x(t)$, while passing the filtered version of the difference-frequency component; that is,

$$
\begin{aligned}
& e(t)=e_{0}(t)+A K \int_{1}^{t} \sin \left[\theta(u)-\theta^{\prime}(u)\right] h(t-u) d u \\
& t \geq 0
\end{aligned}
$$

where it is assumed that the input is applied at $t=0$. $e_{0}(t)$ is the zero-input response which depends only on the initial conditions existing in the filter circuit at $t=0$. In cases where we have control over these initial conditions we generally set them equal to zero, so that $e_{0}(t) \equiv 0$. The veighting function $h(t)$ is known as the impulse response of the filter.

The output frequency of the VCo is

$$
\frac{d \theta^{\prime}(t)}{d t}=\omega_{0}+k_{2} e(t)
$$


where $\omega_{0}$ is the quiescent frequency of the vco.

Assuming that the filter has zero initial conditions;

that is $e_{0}(t)=0$, equations (2.1), (2.2) and (2.3) combine to give

$$
\frac{d \theta^{\prime}(t)}{d t}=\omega_{0}+A K_{1} K_{2} \int_{0}^{t} \sin \left[\theta(u)-\theta^{\prime}(u)\right] h(t-u) d u \text {. }
$$

Now if we define the phase error

$$
\phi(t)=\theta(t)-\theta^{\prime}(t)
$$

and the loop gain

$$
\mathrm{K}=\mathrm{K}_{1} \mathrm{~K}_{2}
$$

we have

$$
\frac{d \phi(t)}{d t}=\frac{d \theta(t)}{d t}-\omega_{0}-A K \int_{0}^{t} h(t-u) \sin \phi(u) d u .
$$

For a given input phase $\theta(t)$, the solution $\phi(t)$ to the integro-differential equation (2.4) describes exactly the operation of the PLL.

In order to avoid repeating the constant $\omega_{0}$ throughout the analysis, one may define the input phase and the vco phase relative to the VCo center frequency as

$$
\begin{aligned}
& \theta_{1}(t)=\theta(t)-\omega_{0} t \\
& \theta_{2}(t)=\theta^{\prime}(t)-\omega_{0} t .
\end{aligned}
$$

Then

$$
\phi(t)=\theta_{1}(t)-\theta_{2}(t)
$$

and (2.4) reduces to 


$$
\frac{d \phi(t)}{d t}=\frac{d \theta(t)}{d t}-A K \int_{0}^{t} h(t-u) \sin \phi(u) d u
$$

which can be modejed as shown in Fig. 3.

Additive noise, $n(t)$, at the input is assumed to be a. narrowband stitionary gausian process with zero-mean and two-sided spectral density $\frac{\mathrm{N}_{0}}{2}$, centered about the signal frequency wo. $n(t)$ has the following narrow-band noise representation [8] :

$$
n(t)=\sqrt{2}\left\{n_{1}(t) \sin \omega_{0} t+n_{2}(t) \cos \omega_{0} t\right\}
$$

where $n_{1}(t)$ and $n_{2}(t)$ are independent gaussiar processes with zero-mean and identical spectral densities, which are the same as the spectral density of $n(t)$ but txanslated downard in frequency so as to be centered about zero frem quency. A derivation similar to the derivation of (2.5) is avaiable in [3] and [17] with the noise having the form

$$
n^{\prime}(t)=-n_{1}(t) \sin \theta_{2}(t)+n_{2}(t) \cos \theta_{2}(t)
$$

where $n^{\prime}(t)$ may be treated as a white gausian random process with spectral density $\frac{N_{0}}{2}$. This leads to the model of the PLL in Fig. 4. The equation of operation of the PLL is now given by

$$
\frac{d \phi(t)}{d t}=\frac{d \theta(t)}{d t}-K \int_{0}^{t} h(t-u)\left[\operatorname{Asin} \phi(u)+n^{\prime}(u)\right] d u \text {. }
$$




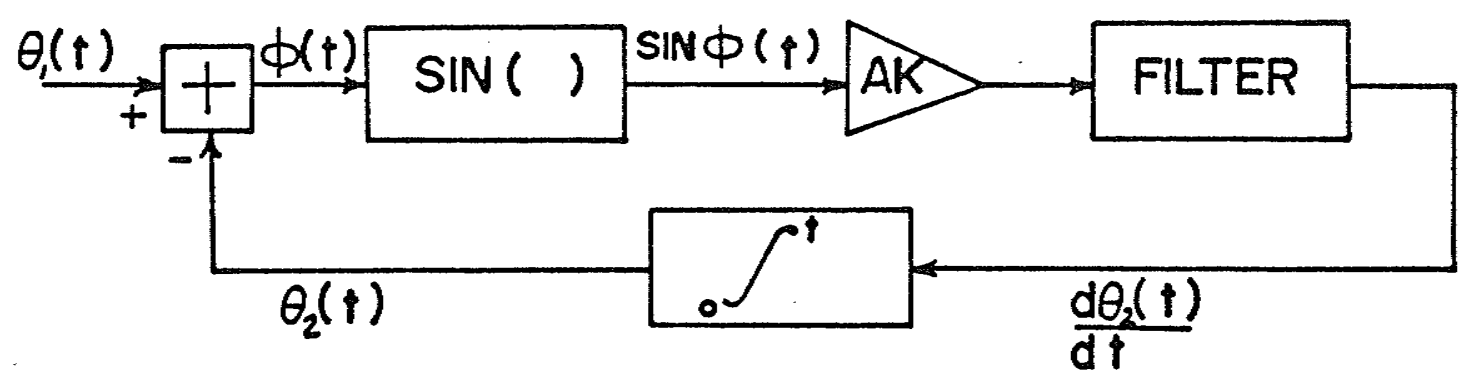

Figure 3. Phase-Locked Loop Modeled Without Noise

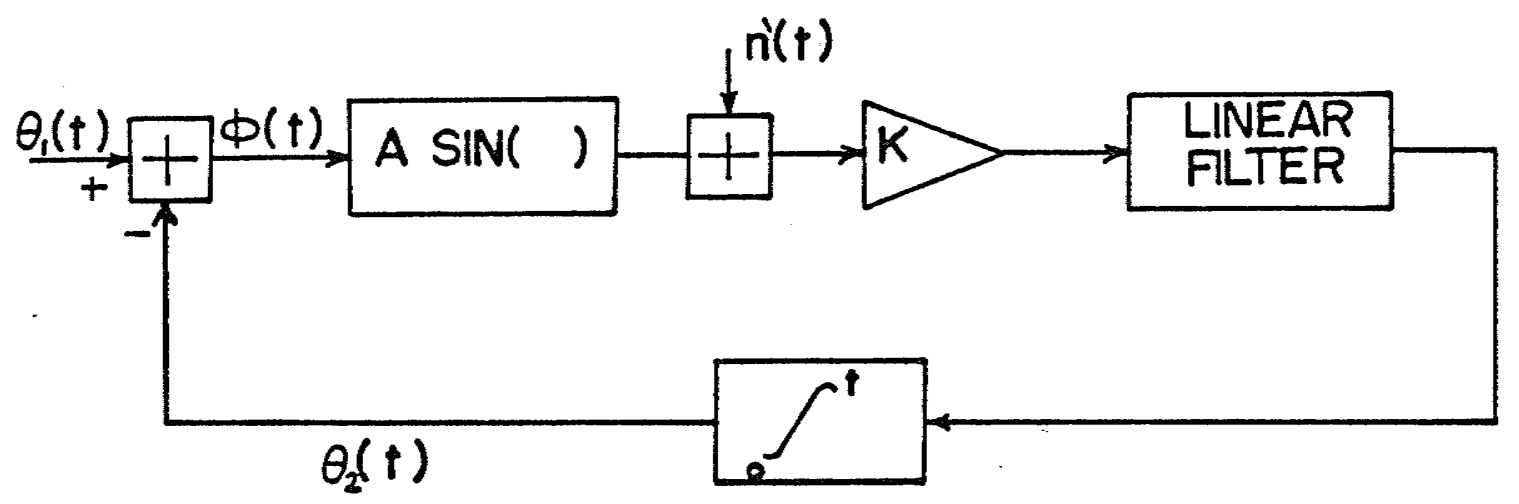

Figure 4. Phase-Locked Loop Modeled With Additive Noise

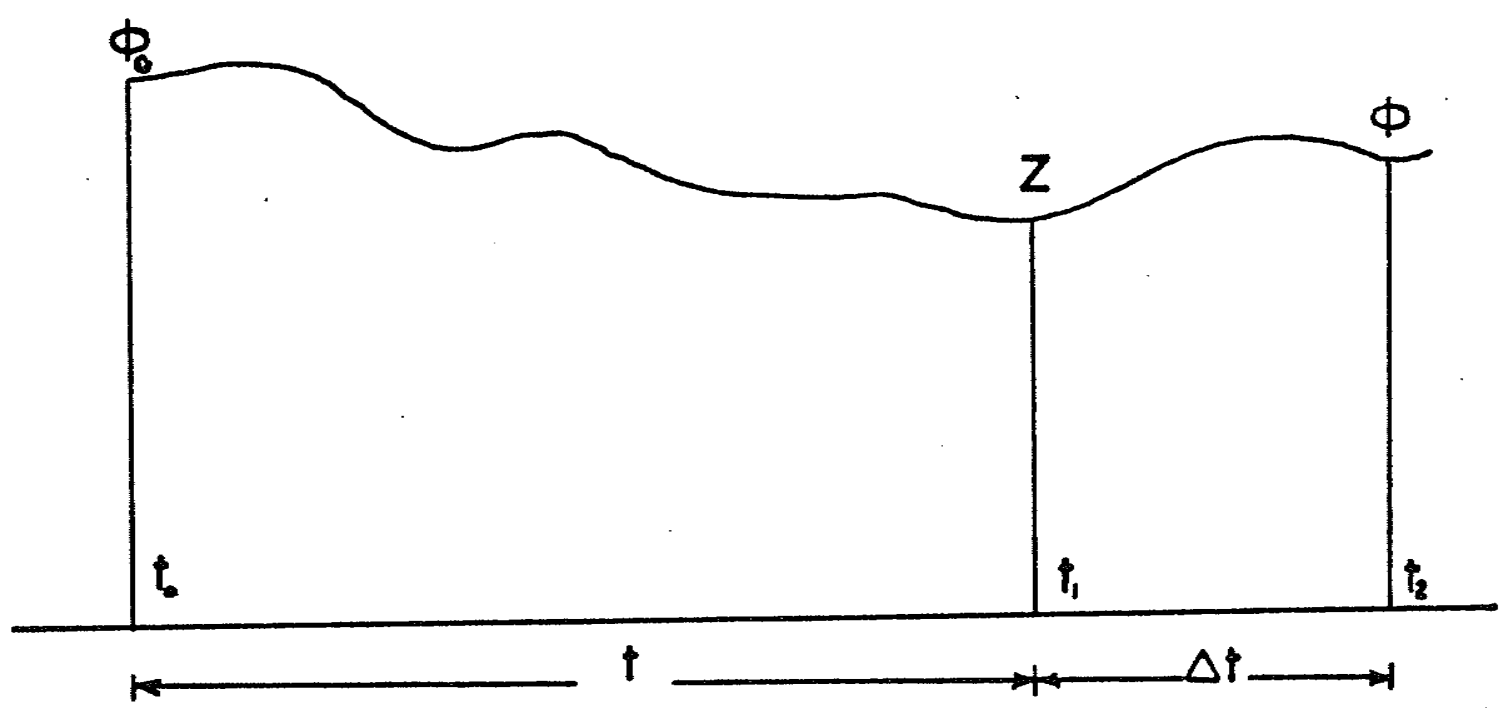

Figure 5. Continuous Markov Process 


\section{$2.3 \quad$ Eokker-Planck Equation}

In this section the effect of stationary white gausian noise will be treated. Before proceeding one should define a Markov process. A process is said to be Markov if the transition-probability distribution (or density function) is a function only of the present value of the process and not of its past values. It should be noted that physical processes described by a differential equation with a white gausian driving function will genera11y be Markov, provided the equation is of the first-order.

\subsubsection{Relations Governing PDF of Markov Processes}

Let $p(\phi, t)$ be the PDF of the phase error $\phi(t)$ after the initial application of the signal. This quantity is, of course, a function of the initial condition, namely the phase error at time $t=0$, which is denoted $\phi_{0}$. It is possible that its initial PDF $\mathrm{p}(\phi, 0)$ is given. This description includes the possibility of a deterministic initial condition, in which case the initial density function becomes

$$
p(\phi, 0)=\delta\left(\phi-\phi_{0}\right)
$$

where $\delta()$ is the Dirac delta function. Henceforth, the conditional probability density is denoted by $p\left(\phi \mid \phi_{0}, t\right)$. The quantity $p\left(\phi \mid \phi_{0}, t\right) d \phi$ is the probability that the value of the process lies in the infinitesimal interval between $\phi$ and $\phi+d \phi$, given that $t$ seconds ago its value was $\phi_{0}$. Consider the continuous Markov process of 
Fig. 5 [3], where $t$ and $\Delta t$ are arbitrary and the values of the process at the three instants $t_{0}, t_{1}$ and $t_{2}$ are $\phi_{0}, z$, and $\phi$ respectively. Since the transition probability at $t_{1}$ depends only on $z$, it follows that the probability density at $t_{2}$. is independent of the value $\phi_{0}$ at $t_{0}$. Thus

$$
p\left(\phi \mid z, \Delta t ; \phi_{0}, t+\Delta t\right)=p(\phi \mid z, \Delta t)
$$

By the definition of a Markov process, the joint probability density of the three samples of the process may be denoted $p\left(\phi, z, \phi_{0}\right)$ after omitting the relative times. Then

$$
\begin{aligned}
\mathrm{p}\left(\phi, z, \phi_{0}\right) & =\mathrm{p}\left(z, \phi_{0}\right) \mathrm{p}\left(\phi \mid z, \phi_{0}\right) \\
& =\mathrm{p}\left(z, \phi_{0}\right) \mathrm{p}(\phi \mid z)
\end{aligned}
$$

Integrating both sides of $(2,8)$ with respect to $z$ and dividing by $\mathrm{p}\left(\phi_{0}\right)$, one obtains after inserting the relative times,

$$
p\left(\phi \mid \phi_{0}, t+\Delta t\right)=\int_{-\infty}^{\infty} p\left(z \mid \phi_{0}, t\right) p(\phi \mid z, \Delta t) d z \quad .
$$

Equation (2.9) is the fundamental relation on the conditional density function of a Markov process. It is called the Smoluchowski equation (or the Chapman-Kolmogorov equation). From (2.9) a partial differential equation in terms of $p(\phi, t)$ can be derived [3] and [19]. The derivation is given in Appendix H.

$$
\frac{\partial p\left(\phi \mid \phi_{0}, t\right)}{\partial t}=\sum_{n \geq 1} \frac{(-1)^{n}}{n !} \frac{\partial^{n}}{\partial \phi^{n}}\left[D_{n}(\phi) p\left(\phi \mid \phi_{0}, t\right)\right]
$$


where

$$
\begin{aligned}
D_{n}(\phi) & =\operatorname{Lim}_{\Delta t \rightarrow 0} \frac{1}{\Delta t} \int_{-\infty}^{\infty}(\Delta z)^{n} p(\Delta z \mid z) d \Delta z \\
& =\operatorname{Lim}_{\substack{\Delta t \rightarrow 0}} \frac{\left.E\left[(\Delta z)^{n}\right\rfloor z\right]}{\Delta t}
\end{aligned}
$$

Equation (2.10) is called the Fokker-Planck equation.

It is shown [20] that the Fokker-Planck equation (2.10) applies equaliy to the more general conditional probability density $p\left(\phi \mid s\left(\phi_{0}\right), t\right)$, where $S\left(\phi_{0}\right)$ is the initial distribution of $\phi$ at $t=0$. This result follows from the fact that the derivation of (2.10) from (2.9) [3] remains valid if $\phi_{0}$ is replaced by $S\left(\phi_{0}\right)$.

The initial condition for the Fokker-Planck equation can be obtained as follows. When $t=0$, $p$ equals the given initial probability density. Thus, in (2.10) when $p(\phi, t)$ is an abbreviation for $p\left(\phi \mid s\left(\phi_{0}\right), t\right)$, the appropriate initial condition is

$$
p(\phi, 0)=S\left(\phi_{0}\right)
$$

on the other hand, when $p(\phi, t)$ is an abbreviation for $p\left(\left.\phi\right|_{0}, t\right)$, the appropriate initial condition is

$$
p(\phi, 0)=\delta\left(\phi-\phi_{0}\right)
$$

where $\delta\left(\phi-\phi_{0}\right)$ is a Dirac delta function.

Hence, we may wite (2.10) in the form

$$
\frac{\partial p(\phi, t)}{\partial t}=\sum_{n=1}^{\infty} \frac{(-1)^{n}}{n !} \frac{\partial^{n}}{\partial \phi^{n}}\left[D_{n}(\phi) p(\phi, t)\right]
$$


keeping in mind the appropriate initial condition.

\subsubsection{Fokker-Planck Equation for the First order PLL}

Consider the nonlinear model in the presence of noise derived in Sec. 2.2, Fig. 3, where the loop filter has unity transfer function and the VCO has a quiescent frequency $\omega_{0}$. At the instant $t=0$ one applies a signal with an amplitude A and frequency $w$ which is close to the quiescent frequency $w_{0}$ of the oscillator. Additive noise, $n(t)$, at the front end of the receiver is a zero-mean gausian process whose spectral density is essentially flat over the frequency range of the receiver and may be assumed white with value $\frac{\mathrm{N}_{0}}{2}$. The equation describing the operation from (2.6) is

$$
\frac{d \phi(t)}{d t}=\left(\omega-\omega_{0}\right)-K\left[\operatorname{Asin} \phi(t)+n^{\prime}(t)\right] .
$$

The noise $n^{\prime}(t)$ is a stationary gaussian process with zeromean and it is nearly white over the frequency range of interest. Therefore, it is concluded that the solution of (2.15) is a Markov process [3] and [18]. Hence, the phase error probability density function should satisfy equation (2.14). From (2.15) one obtains

$$
\begin{aligned}
\Delta \phi(t) & =\phi(t+\Delta t)-\phi(t) \\
& =\left[\left(\omega-\omega_{0}\right)-A K \sin \phi(t)\right] \Delta t-K \int_{t}^{t+\Delta t} n^{\prime}(u) d u .
\end{aligned}
$$

Introducing $(2.16)$ into $(2.11)$ yields,

$$
D_{1}(\phi)=\left(\omega-\omega_{0}\right)-\operatorname{AK} \sin \phi,
$$




$$
\mathrm{D}_{2}(\phi)=\frac{\mathrm{K}^{2} \mathrm{~N}}{2}
$$

and

$$
\mathrm{D}_{\mathrm{n}}(\phi)=0
$$

Equation (2.19) is valid tor all processes which satisFy a first-order ordinary differential equation with a white gaussian driving function, that is, a Markov process.

$$
\begin{aligned}
& \text { Substitution of }(2.17)-(2.19) \text { into }(2.14) \text { yie1ds } \\
& \frac{\partial p(\phi, t)}{\partial t}=-\frac{\partial}{\partial \phi}\left[\left(\omega-\omega_{0}-A K \sin \phi\right) p(\phi, t)\right]+\frac{K^{2} N_{0}}{4} \frac{\partial^{2} p(\phi, t)}{\partial \phi^{2}} .
\end{aligned}
$$

It is convenient to express $(2.20)$ in normalized form by letting

$$
\begin{array}{ll}
B_{I}=\frac{A K}{4} & \begin{array}{l}
\text { Loop-noise bandwidth } \\
\alpha=\frac{A^{2}}{N_{0} B_{L}}
\end{array} \\
\gamma=\frac{\omega-\omega}{4 B_{L}} & \begin{array}{l}
\text { Signal-to-noiseratio } \\
\text { in the loop bandwidth }
\end{array} \\
\tau=4 B_{L} t & \text { Netuning factor } \\
B=1 / \alpha & \text { Notse-to-signal ratio } \\
\text { in the loop bandwidth . (2.21) }
\end{array}
$$

Substituting (2.21) into (2.20) yields the normalized FokkerPlanck equation

$$
\frac{\partial p(\phi, \tau)}{\partial \tau}=\frac{\partial}{\partial \phi}[(\sin \phi-\gamma) p(\phi, \tau)]+\beta \frac{\partial^{2} p(\phi, \tau)}{\partial \phi^{2}} .
$$


Solution of (2.22) with the appropriate initial condition yields a complete statistical description of the process $\phi(t)$. However, an analytic solution of (2.22) has never been found. 


\section{$3.1 \quad$ Introduction}

The result of greatest interest is $P(\Phi, \tau)$, where $\Phi$

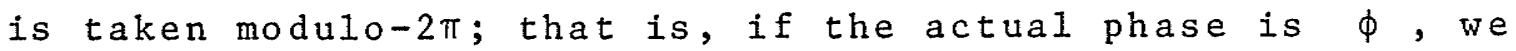
consider instead a phase of $\Phi=\phi-2 n \pi$, where $n$ is an integer such that $|\Phi| \leq \pi$.

The reason for this unusual definition of phase lies in the unfortunate mathematical properties of the phase error variance. Because there is some finite, albeit very sma11, probability of skipping cycles (phase-jumps) if any (gaussian) noise is present, an infinite number of cycles will have been skipped after an infinite time. Therefore, because the averaging interval for determining mean-square phase error, in the steady state, must be infinite to be mathematically correct, the rigorous application of the conventional definition of phase error leads to an infinite answer. The definition of phase (modulo-2T) avoids this mathematical difficulty. Furthermore, most laboratory phase-meters have a range of only $2 \pi$ radians.

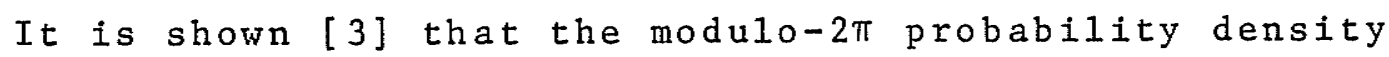
function, $P(\Phi, \tau)$, where

$$
P(\Phi, \tau)=\sum_{k=-\infty}^{\infty} p(\Phi+2 \pi k, \tau) \quad|\Phi| \leq \pi
$$

satisfies the Fokker-Planck equation 


$$
\frac{\partial P(\Phi, \tau)}{\partial \tau}=\frac{\partial}{\partial \Phi}[(\sin \Phi-\gamma) P(\Phi, \tau)]+\beta \frac{\partial^{2} P(\Phi, \tau)}{\partial \Phi^{2}}
$$

Also, $P(\Phi, \tau)$ must be periodic in $\Phi$, with period $2 \pi$, since for any integer m

$$
\begin{aligned}
\mathrm{P}(\Phi+2 \pi \mathrm{m}, \tau) & =\sum_{\mathrm{k}=-\infty}^{\infty} \mathrm{p}[\Phi+2 \pi(\mathrm{m}+\mathrm{k}), \tau] \\
& =\sum_{\mathrm{n}=-\infty}^{\infty} \mathrm{p}(\Phi+2 \pi \mathrm{n}, \tau) \\
& =\mathrm{P}(\Phi, \tau)
\end{aligned}
$$

Therefore, we may solve (3.2) over the interval of just one period, $|\Phi| \leq \pi$, with the normalizing condition

$$
\int_{-\pi}^{\pi} P(\Phi, \tau) d \Phi=1 \quad \text { for a } 11 \tau
$$

and with the appropriate initial condition that will be discussed in sec.(3.2.1). The initial conditions to be considered are

$$
P(\Phi, 0)=\frac{1}{2 \pi} \quad|\Phi| \leq \pi
$$

and

$$
P(\Phi, 0)=\delta\left(\Phi-\Phi_{0}\right) \quad|\Phi| \leq \pi
$$

where $\delta()$ is the Dirac delta function and $\Phi_{0}$ is the initial phase error.

A solution of $(3.2)$ is sought and will lead to a statistical description of the first-order PLL. 
3.2 Fourier Series Expansion of the Phase Error Probability Density Function

Based on the fact that $P(\Phi, \tau)$ is periodic, a Fourier series with time varying coefficients can be used to represent $P(\Phi, \tau)$ in the interval $\Phi[-\pi, \pi]$. That is,

$$
P(\Phi, \tau)=\frac{1}{2} a_{0}(\tau)+\sum_{n \geq 1}\left[a_{n}(\tau) \cos n \Phi+b_{n}(\tau) \sin n \Phi\right]
$$

Hence, the phase error variance $\sigma_{\Phi}^{2}(\tau)$ defined as

$$
\sigma_{\Phi}^{2}(\tau)=\int_{-\pi}^{\pi} \Phi^{2} P(\Phi, \tau) d \Phi
$$

will be

$$
\sigma_{\Phi}^{2}(\tau)=\frac{\pi^{2}}{3}+4 \pi \sum_{n \geq 1} \frac{(-1)^{n} a_{n}(\tau)}{n^{2}} .
$$

Determination of the Fourier series coefficients $a_{n}(\tau)$, $b_{n}(\tau)$ and $a_{0}(\tau)$ leads to the solution of $P(\Phi, \tau)$ and $\sigma_{\Phi}^{2}(\tau)$

The coefficient $a_{0}(\tau)$ can be determined directly by applying the normalizing condition ( 3.3 ) which yields,

$$
a_{0}(\tau)=\frac{1}{\pi}
$$

Introducing (3.6) into the Fokker-Planck equation (3.2) and omitting the time $\tau$ for the moment yields, 


$$
\begin{aligned}
& \sum_{n \geq 1}\left(\dot{a}_{n} \cos n \Phi+\dot{b}_{n} \sin n \Phi\right)=(\sin \Phi-\gamma) \sum_{n \geq 1}\left(-n a_{n} \sin n \Phi+n b b_{n} \cos n \Phi\right) \\
& +\cos \Phi\left[\frac{1}{2} a_{0}+\sum_{n \geq 1}\left(a_{n} \cos n \Phi+b_{n} \sin n \Phi\right)\right] \\
& -\beta \sum_{n \geq 1}\left(n^{2} a_{n} \cos n \Phi+n^{2} b_{n} \sin n \Phi\right) \\
& =\sum_{n \geq 1}-n a_{n} \sin \Phi \cdot \sin n \Phi+\sum_{n \geq 1} n b_{n} \sin \Phi \cdot \cos n \Phi \\
& +\gamma \sum_{n \geq 1} n a_{n} \sin n \Phi-\gamma \sum_{n \geq 1} n b_{n} \cos n \Phi \\
& +a_{0} \cos \Phi+\sum_{n \geq 1} a_{n} \cos \Phi \cdot \cos n \Phi \\
& +\sum_{n \geq 1} b_{n} \cos \Phi \cdot \sin n \Phi-\beta \sum_{n \geq 1} n^{2} a_{n} \cos n \Phi \\
& -\beta \sum_{n \geq 1} n^{2} b_{n} \operatorname{sinn} \Phi
\end{aligned}
$$

Making use of the trigenometric identities,

$$
\begin{aligned}
& \sin x \cdot \operatorname{sinn} x=\frac{1}{2}[\cos (n-1) x-\cos (n+1) x] \\
& \cos x \cdot \cos n x=\frac{1}{2}[\cos (n-1) x+\cos (n+1) x] \\
& \sin x \cdot \cos n x=\frac{1}{2}[\sin (n+1) x-\sin (n-1) x] \\
& \cos x \cdot \operatorname{sinnx}=\frac{1}{2}[\sin (n+1) x+\sin (n-1) x]
\end{aligned}
$$

and combining the similar terms in (3.10) yields, 


$$
\begin{aligned}
\sum_{n \geq 1}\left(\dot{a}_{n} \cos n \Phi+\dot{b}_{n} \sin n \Phi\right) & =-\sum_{n \geq 1} \frac{n-1}{2} a_{n} \cos (n-1) \Phi+\sum_{n \geq 1} \frac{n+1}{2} a_{n} \cos (n+1) \Phi \\
& -\gamma \sum_{n \geq 1} n b_{n} \cos n \Phi+\frac{a_{0}}{2} \cos \Phi-\beta \sum_{n \geq 1} n^{2} a_{n} \cos n \Phi \\
& -\sum_{n \geq 1} \frac{n-1}{2} b_{n} \sin (n-1) \Phi+\sum_{n \geq 1} \frac{n+1}{2} b_{n} \sin (n+1) \Phi \\
& +\gamma \sum_{n \geq 1} n a_{n} \operatorname{sinn} \Phi-\beta \sum_{n \geq 1} n^{2} b_{n} \operatorname{sinn} \Phi .
\end{aligned}
$$

After appropriate redefinitions of the dummy index in certain of the sums, (3.11) becomes

$$
\begin{aligned}
\sum_{n \geq 1}\left(\dot{a}_{n} \cos n \Phi+\dot{b}_{n} \sin n \Phi\right) & =\sum_{n=0}^{\infty} \frac{-n}{2} a_{n+1} \cos \Phi+\sum_{n \geq 2} \frac{n}{2} a_{n-1} \cos n \Phi \\
& -\gamma \sum_{n \geq 1} n b_{n} \cos n \Phi-\beta \sum_{n \geq 1} n^{2} a_{n} \cos n \Phi+\frac{a}{2} \cos \Phi \\
& -\sum_{n=0}^{\infty} \frac{n}{2} b_{n+1} \sin n \Phi+\sum_{n \geq 2} \frac{n}{2} b_{n-1} \operatorname{sinn} \Phi \\
& +\gamma \sum_{n \geq 1} n a_{n} \sin \Phi-\beta \sum_{n \geq 1} n^{2} b_{n} \sin n \Phi
\end{aligned}
$$

Equating the coefficients of each harmonic in (3.12) yields,

$$
\begin{aligned}
& \dot{a}_{n}=\frac{n}{2} a_{n-1}-\beta n^{2} a_{n}-\frac{n}{2} a_{n+1}-\gamma n b_{n}, \\
& \dot{b}_{n}=\frac{n}{2} b_{n-1}-\beta n^{2} b_{n}-\frac{n}{2} b_{n+1}+\gamma a_{n} \quad, \quad n=1,2,3, \ldots
\end{aligned}
$$

and

$$
a_{0}=\frac{1}{\pi} \quad \text { and } \quad b_{0}=0 \quad \text {. }
$$


Equations ( 3.13 ) constitute two coupled systems of simultaneous differential equations of infinite dimension; their solution yields $a_{n}(\tau)$ and $b_{n}(\tau)$.

\subsection{Initial Conditions}

In this study we consider two cases of initial conditions.

\subsubsection{Deterministic Case}

When the initial phase error at time $\tau=0$ is known, it will be denated by $\Phi_{0}$, hence

$$
P(\Phi, 0)=\delta\left(\Phi-\Phi_{0}\right) \quad
$$

The corresponding initial conditions of the Fourier coefficients $a_{n}(0)$ and $b_{n}(0)$ can be determined as follows. Let $q_{n}\left(\Phi-\Phi_{0}\right)$ be periodic pulse train of period $2 \pi$ and duration $2 \mathrm{~h}$, where $\mathrm{h}<\pi / 2$. Consider only one period as shown in Fig. 6 .

$$
\begin{array}{ll}
\mathrm{q}\left(\Phi-\Phi_{0}\right)=0 & \left|\Phi-\Phi_{0}\right|>\mathrm{h} \\
\mathrm{q}\left(\Phi-\Phi_{0}\right)=\frac{1}{2 h} & \left|\Phi-\Phi_{0}\right|<\mathrm{h}
\end{array}
$$

and

$$
\int_{-\pi}^{\pi} q\left(\Phi-\Phi{ }_{0}\right) d \Phi=1
$$

Since $q$ is periodic, a Fourier series can be used to represent $q$ in the interval $[-\pi, \pi]$; that is, 


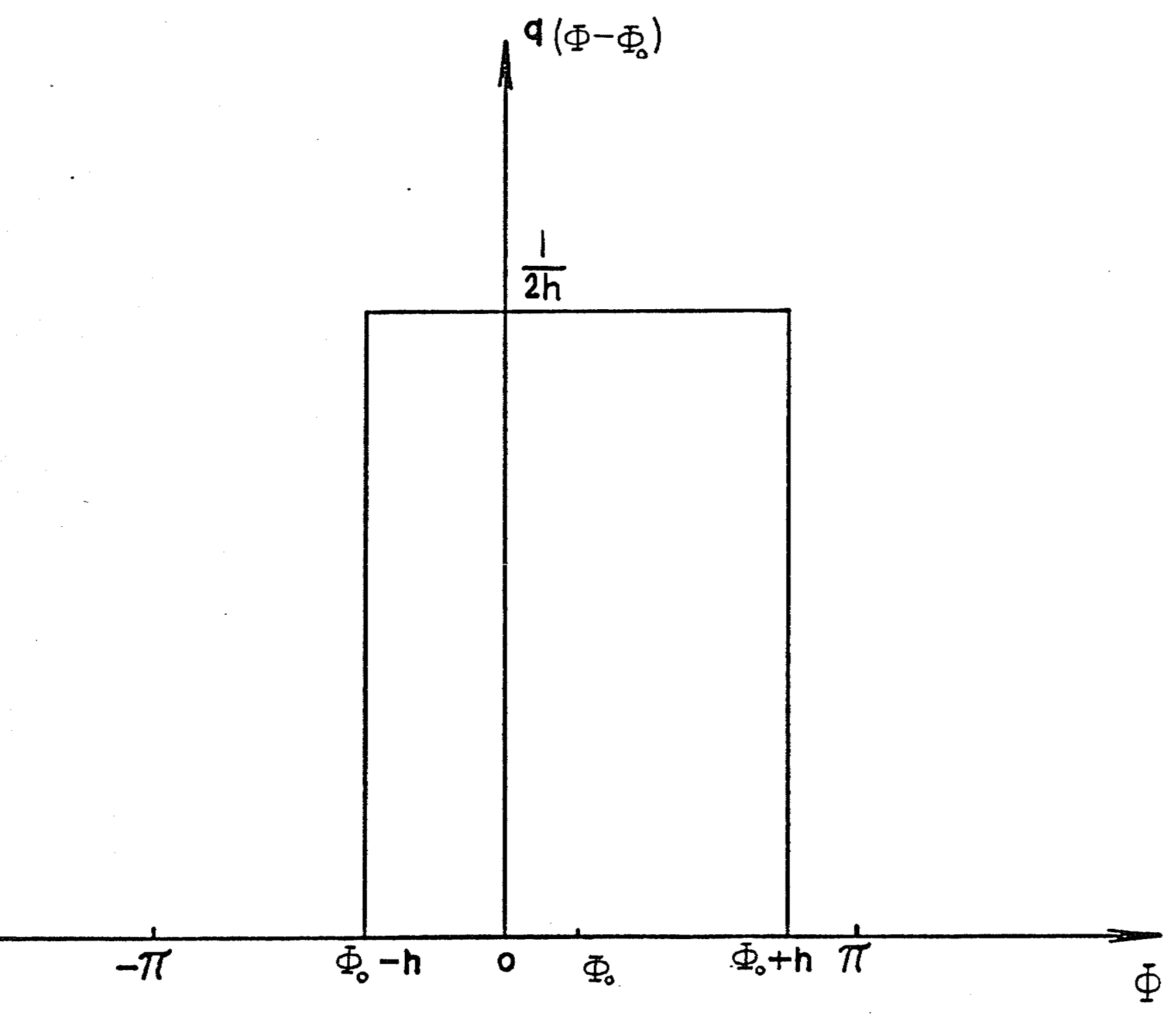

Figure 6. Dirac Delta Function Representation 


$$
q(\Phi-\Phi)=\frac{1}{2} \hat{a}_{0}+\sum_{n \geq 1}\left(\hat{a}_{n} \cos n \Phi+\hat{b}_{n} \sin n \Phi\right)
$$

where

$$
\begin{aligned}
\hat{a}_{0} & =\frac{1}{\pi} \int_{-\pi}^{\pi} q\left(\Phi-\Phi_{0}\right) d \Phi \\
& =\frac{1}{\pi} \int_{\Phi}^{\Phi}{ }_{0}+h \frac{1}{2 h} d \Phi=\frac{1}{\pi} \\
\hat{a}_{n} & =\frac{1}{\pi} \int_{-\pi}^{\pi} q\left(\Phi-\Phi_{0}\right) \cos n \Phi d \Phi \\
\hat{a}_{n} & =\frac{1}{\pi} \int_{0}^{\Phi+h} \frac{1}{2 h} \cos n \Phi d \Phi \\
0 & =\frac{1}{\pi} \cos n \Phi \frac{s 1 n}{n h}
\end{aligned}
$$

and

$$
\begin{aligned}
\hat{\mathrm{b}}_{\mathrm{n}} & =\frac{1}{\pi} \int_{-\pi}^{\pi} \mathrm{q}\left(\Phi-\Phi_{0}\right) \operatorname{sinn} \Phi \mathrm{d} \Phi \\
& =\frac{1}{\pi} \int_{\Phi_{0}-\mathrm{h}}^{\Phi}{ }_{0}^{+h} \frac{1}{2 \mathrm{~h}} \operatorname{sinn} \Phi \mathrm{d} \Phi \\
& =\frac{1}{\pi} \operatorname{sinn} \Phi 0_{0} \frac{\sin \mathrm{nh}}{\mathrm{nh}}
\end{aligned}
$$

The Dirac delta function can be defined as

$$
\delta\left(\Phi-\Phi_{0}\right)=\operatorname{Lim}_{h \rightarrow 0} q\left(\Phi-\Phi_{0}\right)
$$




$$
\begin{aligned}
& =\frac{1}{2 \pi}+\frac{1}{\pi} \operatorname{Lim}_{h \rightarrow 0} \sum_{n \geq 1}\left(\cos n \Phi{ }_{0} \frac{\sin n h}{n h} \cos n \Phi+\sin \Phi_{0} \frac{\sin n h}{n h} \sin \Phi\right) \\
& =\frac{1}{2 \pi}+\frac{1}{\pi} \sum_{n \geq 1}\left(\cos \Phi_{0} \cos n \Phi+\operatorname{sinn} \Phi_{0} \sin n \Phi\right) \\
& =\frac{1}{2 \pi}+\frac{1}{\pi} \sum_{n \geq 1} \cos n\left(\Phi-\Phi_{0}\right)
\end{aligned}
$$

Hence

$$
a_{n}(0)=\frac{1}{\pi} \cos n \Phi_{0}
$$

and

$$
\mathrm{b}_{\mathrm{n}}(0)=\frac{1}{\pi} \operatorname{sinn} \Phi_{0}
$$

The Fourier series expansion of the Dirac delta function is discussed in [10], [23], [24] and [31].

\subsubsection{Arbitrary Initial Phase Distribution}

If the initial phase error at time $\tau=0$ is complete1y unknown, it will be assumed to have a uniform distribution; that is,

$$
P(\Phi, 0)=\frac{1}{2 \pi} \quad, \quad|\Phi| \geq \pi \quad \text {. }
$$

The corresponding initial conditions of the Fourier coefficients, $a_{n}(0)$ and $b_{n}(0)$, will be

$$
\begin{aligned}
& a_{n}(0)=0 \\
& b_{n}(0)=0
\end{aligned}
$$


3.4 Computation of the Fourier Series Coefficients

To compute the Fourier series coefficients ( $a_{n}(\tau)$ and $\left.b_{n}(\tau)\right)$ the two coupled systems of infinite dimension (3.13) have to be solved with the fnitial conditions discussed in sec. 3.3 .

Unfortunately, a general closed form solution of (3.13) cannot be obtained. However for some special cases an analytic solution is obtained in sec. 3.4.1. An analytic approximate solution based on the successive approximation method is given in Sec. 3.4.2.

It should be mentioned that the Fourier coefficients satisfy the Bessel inequality [21]

$\frac{a^{2}}{2}+\sum_{n \geq 1}\left(a_{n}^{2}(\tau)+b_{n}^{2}(\tau)\right) \leq \frac{1}{\pi} \int_{-\pi}^{\pi} P^{2}(\Phi, \tau) d \Phi$

for a $11 \quad \tau>0$

However, since $P(\Phi, \tau)$ is a continuous, finite function of $\Phi$ for $\tau>0$, so is $P^{2}(\Phi, \tau)$, and thus, the integration in ( 3.24 ) exists and is finite. Furthermore, the Bessel inequality (3.24) implies that

$$
\lim _{n^{\rightarrow \infty}} a_{n}(\tau)=0
$$

and

$$
\lim _{n \rightarrow \infty} b_{n}(\tau)=0 \quad \text { for fixed } \tau
$$

Equations (3.25) together with the truncation justification discussed in Appendix I indicate that the infinite system of equations 
(3.13) can be truncated and hence, $P(\Phi, \tau)$ and $\sigma_{\Phi}^{2}(\tau)$, from (3.6) and (3.8) respectively, can be calculated with consjderable accuracy by using only a few terms of the expansion. Therefore, a solution of the two coupled systems (3.13) with finite dimension is sought. This is done by simulation on a digital computer. The results are discussed in Chapter $V$. A solution can also be obtained by implementing any of the well established techniques used for the analysis of RLC ladder networks [33]-[40]. The ladder network representation of the problem is given in Sec. 3.5 .

\subsubsection{Analytic solutions for the Fourier Coefficients (Special Cases)}

Analytic solutions of the infinite systems (3.13) for two special cases are presented. These cases correspond to two different modes of operation of the PLL. It is assumed in both cases that there is no additive noise with the incoming signal; that is, $\beta=0$.

3.4 .1 a case of $\beta=0$ and $\gamma=0$

This is the case when the frequency of the incoming signal is known so that the VCO quiescent frequency can be set accordingly; consequently the problem is merely one of tracking the phase. The solution is sought for the case where the inftial phase error is completely unknown and is therefore assumed to have a uniform distribution; that 1 , $P(\Phi, 0)=\frac{1}{2 \pi},|\Phi| \leq \pi$ and zero elsewhere. This is equivalent to $a_{n}(0)=b_{n}(0)=0$. 
For this special case the infinite systems (3.13) be-

come

$$
\dot{a}_{n}(\tau)=\frac{n}{2} a_{n-1}(\tau)-\frac{n}{2} a_{n+1}(\tau)
$$

and

$$
\dot{b}_{n}(\tau)=\frac{n}{2} b_{n-1}(\tau)-\frac{n}{2} b_{n+1}(\tau) \quad n=1,2,3, \ldots
$$

with

$$
a_{0}=\frac{I}{\pi} \quad, \quad b_{0}=0,
$$

and

$$
a_{n}(0)=b_{n}(0)=0
$$

Since $b_{0}=0$ and $b_{n}(0)=0$ it is evident that

$$
b_{n}(\tau)=0 \quad \text { for a } 11 \quad \tau \geq 0 \text {. }
$$

To determine $a_{n}(\tau)$, a solution of the form.

$$
a_{K}(\tau)=\sum_{r=0}^{\infty} g_{r}(\tau) K^{(r)}
$$

is attempted. Where $\mathrm{K}^{(\mathrm{r})}$ is defined as

$$
\begin{aligned}
\mathrm{K}^{(\mathrm{r})} & =\mathrm{K}(\mathrm{K}-\mathrm{I})(\mathrm{K}-2) \ldots(\mathrm{K}-\mathrm{r}+1) \\
& =\frac{\Gamma(\mathrm{K}+1)}{\Gamma(\mathrm{K}-\mathrm{r}+1)}
\end{aligned}
$$

Noticing that when $\mathrm{K}$ is a nonnegative integer the series (3.29) will obviously terminate with $\mathrm{K}+1$ terms or 1 ess, and thus convergence questions then are not involved. If use is made of the easily established relationship

$$
\mathrm{KK}^{(\mathrm{r})}=\mathrm{K}^{(\mathrm{r}+1)}+\mathrm{rK}^{(\mathrm{r})}
$$

\section{(3.26) can be put in the form}




$$
\sum_{r=0}^{\infty} \dot{g}_{r} K^{(r)}=-\sum_{r=0}^{\infty} r g_{r} K^{(r)}-\sum_{r=0}^{\infty} \frac{r(r+1)}{2} g_{r+1} k(r)
$$

after appropriate redefinitions of the dumy index in certain of the sums. The requirement that the coefficients of all relevant factorial powers of $K$ vanish independently then yields the conditions

$$
\dot{\mathrm{g}}_{0}(\tau)=0
$$

in correspondence with $r=0$, and the recurrence formula

$$
\dot{\mathrm{g}}_{\mathrm{r}}(\tau)=-r \mathrm{~g}_{\mathrm{r}}(\tau)-\frac{r(\mathrm{r}+1)}{2} \mathrm{~g}_{r+1}(\tau) \quad r=1,2,3, \ldots \cdot(3.34)
$$

The initial condition, $g_{r}(0)$, corresponding to $a_{n}(0)$ can be easily evaluated from (3.29). The result is

$$
g_{r}(0)=\frac{(-1)^{r}}{\pi r !}
$$

Equations (3.34) can be written in matrix form as

$$
\dot{\mathrm{g}}=\mathrm{Bg}
$$

where

$$
g=\left[\begin{array}{llllll}
g_{1} & g_{2} & \ldots & g_{r} & \ldots & ]^{T}
\end{array}\right.
$$

and

$$
B=\left[\begin{array}{ccccc}
-1 & -1 & & & \\
& -2 & -3 & & 0 \\
& & -3 & -6 & \\
& & & -r & \frac{-r(r+1)}{2} \\
& & & & \ddots
\end{array}\right]
$$


The matrix $B$ is an upper triangular infinite matrix which according to Cooke [26] has eigenvalues $\lambda_{\mathrm{n}}$ that lie on the main diagona1. Hence

$$
\lambda_{\mathrm{n}}=-\mathrm{n} \quad \text { where } \quad \mathrm{n}=1,2,3, \ldots \ldots
$$

and therefore the solution of $(3.36)$ can be put in the form

$$
g(\tau)=\sum_{n \geq 1} d_{n} U_{n} e^{-n \tau}
$$

where $d_{n}$ 's are constants to be determined from the initial conditions and $U_{n}$ is the eigenvector corresponding to $\lambda_{n}$. As shown in Appendix A the modal matrix; that is, the matrix that has as its columns the eigenvectors, is

$$
\begin{aligned}
Q=\left\{q_{i j}\right\} & =\left\{\frac{2^{i-1}(j-1) !}{i !(i-1) !(j-i) !}\right\} \\
& =\left\{\frac{2^{i-1}}{i !}\left(\begin{array}{c}
j-1 \\
i-1
\end{array}\right)\right\}
\end{aligned}
$$

Hence

$$
g_{r}(\tau)=\frac{2^{r-1}}{r !(r-1) !} \sum_{n=r}^{\infty} \frac{(n-1) !}{(n-r) !} d_{n} e^{-n \tau}
$$

The constants $d_{n}$, determined in Appendix $B$, are

$$
\mathrm{d}_{\mathrm{n}}=(-1)^{\mathrm{n}} \frac{2}{\pi}
$$

By making use of the known relation [27]

$$
\begin{aligned}
& \sum_{n=0}^{\infty}(-1)^{n} z^{n} \frac{\Gamma(n+r)}{n !}=\Gamma(r)(1+z)^{-r} \\
& |z| \geq 1 \quad ; \quad \text { if } \quad r>0, \quad z \neq-1
\end{aligned}
$$


the result of introducing (3.42) into $(3.41)$ is

$$
g_{r}(\tau)=\frac{(-1)^{r}}{\pi r !}\left[\frac{2}{e^{\tau}+1}\right]^{r}
$$

Hence,

$$
a_{K}(\tau)=\frac{1}{\pi} \sum_{r=0}^{\infty}(-1)^{r}\left(\begin{array}{l}
K \\
r
\end{array}\right)\left[\frac{2}{e^{\tau}+1}\right]^{r}
$$

which is the Binomial series of $\frac{1}{\pi}\left(1-\frac{2}{e^{\tau}+1}\right)^{K}$.

Thus,

$$
a_{K}(\tau)=\frac{1}{\pi}[\tanh \tau / 2]^{K}
$$

This result can also be obtained by another method mentioned in Appendix C.

Having solved for the Fourier coefficients, the modulo$2 \pi$ probability density function and the variance of the phase error can be obtained by introducing (3.28) and (3.46) into $(3.6)$ and $(3.8)$ respectively

$$
P(\Phi, \tau)=\frac{1}{2 \pi}+\frac{1}{\pi} \sum_{n \geq 1}[\tanh \tau / 2]^{n} \cos n \Phi,
$$

and

$$
\sigma_{\Phi}^{2}(\tau)=\frac{\pi^{2}}{3}+4 \sum_{n \geq 1} \frac{(-1)^{n}}{n^{2}}[\tanh \tau / 2]^{n}
$$

In Appendix $D$ it is shown that the infinite sum in $(3.47)$ converges to

$$
P(\Phi, \tau)=\frac{1}{2 \pi(\cosh \tau-\sinh \tau \cdot \cos \Phi)}
$$


$3.4 .1 \mathrm{~b}$ Case of $\beta=0$ and $\gamma \neq 0$

In this case the frequency of the incoming signal differs from the quiescent frequency of the VCo; that is, $\gamma \neq 0$. This is a more practical situation. The frequency difference may be due to an actual difference between the transmitter and receiver, or it may be due to a Doppler shift.

The initial phase error is assumed to have a uniform distribution. For this case the infinite systems ( 3.13 ) become

$$
\begin{aligned}
& \dot{a}_{n}(\tau)=\frac{n}{2} a_{n-1}(\tau)-\frac{n}{2} a_{n+1}(\tau)-\gamma n b_{n}(\tau), \\
& \dot{b}_{n}(\tau)=\frac{n}{2} b_{n-1}(\tau)-\frac{n}{2} b_{n+1}(\tau)+\gamma n a_{n}(\tau), n=1,2,3, \ldots
\end{aligned}
$$

and

$$
a_{0}=\frac{1}{\pi} \text { and } \quad b_{0}=0
$$

Based on the solution of the case where $\gamma=0$ and a knowledge of the stationary solution of (3.50), the complete solution of (3.50) with the initial conditions

$$
a_{n}(0)=b_{n}(0)=0
$$

is assumed in the form

$$
a_{n}(\tau)=\frac{1}{\pi}[g(\tau)]^{n} \cos (n f(\tau))
$$

and

$$
b_{n}(\tau)=\frac{1}{\pi}[g(\tau)]^{n} \sin (n f(\tau))
$$

where $f(\tau)$ and $g(\tau)$ are functions of time $\tau$ with initial values

$$
g(0)=f(0)=0
$$


corresponding to $(3.51)$.

Introducing ( 3.52$)$ into (3.50) and omitting the time $\tau$ for the moment yields,

$-g \dot{f} \sin (n f)+\dot{g} \cos (n f)=\frac{1}{2} \cos ((n-1) f)-\frac{g^{2}}{2} \cos ((n+1) f)-\gamma g \sin (n f)$

$g \dot{f} \cos (n f)+\dot{g} \sin (n f)=\frac{1}{2} \sin ((n-1) f)-\frac{g^{2}}{2} \sin ((n+1) f)+\gamma g \cos (n f)$

where

$$
\dot{\mathrm{f}}=\frac{\mathrm{df}}{\mathrm{d} \tau} \quad \text { and } \quad \dot{\mathrm{g}}=\frac{\mathrm{dg}}{\mathrm{d} \tau}
$$

Multiplication of $(3.54)$ by $\cos (n f)$ and $(3.55)$ by $\sin (n f)$ and addition yield (after making use of the trigenometric identity $\quad \cos (x-y)=\cos x \cdot \cos y+\sin x \cdot \sin y) \quad$,

$$
\dot{g}=\frac{1}{2}\left(1-g^{2}\right) \cos f
$$

Also multiplication of (3.54) by $\sin (n f)$ and (3.55) by $\cos (n f)$ and subtracting yield (after making use of the trigenometric identity $\sin (x-y)=\sin x \cdot \cos y-\cos x \cdot \sin y)$,

$$
\dot{\mathrm{f}}=\gamma-\frac{1+\mathrm{g}^{2}}{2 \mathrm{~g}} \sin \mathrm{f}
$$

Solutions of the nonlinear, simultaneous differential equations $(3.56)$ and $(3.57)$ are given in Appendix E.

The results are:

i) For $\gamma<1$

$f(\tau)=\arctan \left\{\frac{\gamma}{\sqrt{1-\gamma^{2}}} \tanh \left(\frac{\tau}{2} \sqrt{1-\gamma^{2}}\right)\right\}$ 
and

$$
g(\tau)=\frac{\sinh \left(\frac{\tau}{2} \sqrt{1-\gamma^{2}}\right)}{\sqrt{\cosh ^{2}\left(\frac{\tau}{2} \sqrt{1-\gamma^{2}}\right)-\gamma^{2}}}
$$

ii) For $\gamma>1$

$$
f(\tau)=\arctan \left\{\frac{\gamma}{\sqrt{\gamma^{2}-1}} \tan \left(\frac{\tau}{2} \sqrt{\gamma^{2}-1}\right)\right.
$$

and

$$
g(\tau)=\frac{\sin \left(\frac{\tau}{2} \sqrt{\gamma^{2}-1}\right)}{\sqrt{\gamma^{2}-\cos ^{2}\left(\frac{\tau}{2} \sqrt{\gamma^{2}-1}\right)}}
$$

iii) For $\gamma=1$

$$
f(\tau)=\arctan \left(\frac{\tau}{2}\right)
$$

and

$$
g(\tau)=\frac{\tau}{\sqrt{4+\tau^{2}}}
$$

Introducing equations $(3.58)-(3.63)$ into $(3.52)$ yields the corresponding Fourier coefficients which are to be substit-

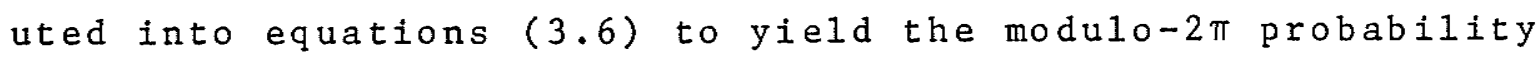
density functions of the phase error in the form of an infinite series for each case. In Appendix $F$ it is shown that these infinite series, for different values of $\gamma$, converge to

$$
\begin{aligned}
& P(\Phi, \tau)=\frac{1}{2 \pi} \frac{1-\gamma^{2}}{\cosh \tau_{1}-\gamma^{2}-\sqrt{1-\gamma^{2}} \sinh \tau_{1} \cdot \cos \Phi+\gamma\left(1-\cosh \tau_{1}\right) \sin \Phi} \\
& \text { where } \tau_{1}=\tau \sqrt{1-\gamma^{2}}, \\
& P(\Phi, \tau)=\frac{1.64)}{\pi\left(\tau^{2}+2-2 \tau \cos \Phi-\tau^{2} \sin \Phi\right)},
\end{aligned}
$$

when $\gamma=1$ 
and

$$
\begin{gathered}
P(\Phi, \tau)=\frac{1}{2 \pi} \frac{\gamma^{2}-1}{\gamma^{2}-\cos \tau_{2}-\sqrt{\gamma^{2}-1} \sin \tau_{2} \cos \Phi+\gamma\left(1-\cos \tau_{2}\right) \sin \Phi} \\
\text { where } \tau_{2}=\tau \sqrt{\gamma^{2}-1} \quad, \quad \gamma>1 \quad .
\end{gathered}
$$

Hence for $\gamma \leq 1, P(\Phi, \tau)$ will tend to its stationary value $P(\Phi, \infty)=\delta\left(\Phi-\Phi_{0}\right), \Phi_{0}$ is the steady state value of the phase error. It is shown in Sec. 3.6 that

$$
\Phi_{0}=\arcsin \gamma
$$

For $\gamma>1$ it follows directly from (3.66) that $P(\Phi, \tau)$ is periodic with respect to $\tau$ and cannot tend to any limit with increasing $\tau$. In this case the phase error is a periodic nonstationary process.

Alternatively, if $\left(\omega-\omega_{0}\right) \leq A K$ the loop locks with a steady state phase error $\Phi_{0}=\arcsin \gamma$. If $\left(\omega-\omega_{0}\right)>A K$. the loop never achieves lock; the phase error continues to increase or decrease forever along a sinusiodal trajectory.

\subsubsection{Approximate Solution in Closed Form}

An approximate solution of (3.13) in closed form can be obtained by the successive approximation method. Without loss in generality, the case when $\gamma=0$ and $P(\Phi, 0)=\delta(\Phi)$ is considered. This is equivalent to solving the system

$$
\dot{a}_{n}(\tau)=\frac{n}{2} a_{n-1}(\tau)-n^{2} B a_{n}(\tau)-\frac{n}{2} a_{n+1}(\tau)
$$

with $\quad a_{0}=\frac{1}{\pi} \quad$ and $\quad a_{n}(0)=\frac{1}{\pi}$. 
Multiplying both sides of (3.68) by $e^{n^{2} \beta \tau}$ and rearranging some of the terms yields,

$$
\frac{d}{d \tau}\left(e^{n^{2} \beta \tau} a_{n}(\tau)\right)=\frac{n}{2} e^{n^{2} \beta \tau}\left(a_{n-1}(\tau)-a_{n+1}(\tau)\right)
$$

By integration ( 3.69 ) becomes

$$
a_{n}(\tau)=\frac{1}{\pi} e^{-n^{2} \beta \tau}+\frac{n}{2} e^{-n^{2} \beta \tau} \int_{0}^{\tau}\left[a_{n-1}(t)-a_{n+1}(t)\right] e^{n^{2} \beta t} d t .
$$

As an initial guess, choosing $a_{n}^{(0)}(\tau)=a_{n}(0)=\frac{1}{\pi}$ to be substituted on the right-hand side of (3.70) to yield, as a first approximation,

$$
a_{n}^{(1)}(\tau)=\frac{1}{\pi} e^{-n^{2} \beta \tau}
$$

Introducing (3.71) into the right-hand side of (3.70) yields, as a second approximation,

$$
\begin{aligned}
a_{n}^{(2)}(\tau)=\frac{1}{\pi} e^{-n^{2} \beta \tau} & +\frac{n}{\beta \pi\left(4 n^{2}-1\right)}\left[(2 n \cosh \varepsilon+\sinh \varepsilon) e^{-\left(n^{2}+1\right) \beta \tau}\right. \\
& \left.-2 n e^{-n^{2} \beta \tau}\right]
\end{aligned}
$$

where $\quad \varepsilon=2 \mathrm{n} B \tau$.

As a third approximation one obtains

$$
\begin{aligned}
a_{n}^{(3)}(\tau) & =\frac{1}{\pi} e^{-n^{2} \beta \tau}+\frac{n}{2 \pi \beta}\left\{\frac{2 e^{-\left(n^{2}+1\right) \beta \tau}}{4 n^{2}-1}(2 n \cosh \varepsilon+\sinh \varepsilon)\right. \\
& -\frac{4 e^{-\left(n^{2}+1\right) \beta \tau}}{\beta\left(4 n^{2}-1\right)^{2}\left(4 n^{2}-9\right)}\left[2 n\left(4 n^{4}-9 n^{2}+4\right) \cosh \varepsilon+\left(4 n^{4}-5 n^{2}+3\right) \sinh \varepsilon\right]
\end{aligned}
$$




$$
\begin{aligned}
& +\frac{e^{-\left(n^{2}+4\right) \beta \tau}}{4 \beta\left(4 n^{2}-9\right)}(2 n \cosh 2 \varepsilon+3 \sinh 2 \varepsilon) \\
& +\frac{3 n e^{-n^{2} \beta \tau}}{\beta\left(4 n^{2}-1\right)^{2}\left(4 n^{2}-9\right)}\left(4 n^{4}-9 n^{2}+4\right) \\
& \left.-\frac{4 n e^{-n^{2} \beta \tau}}{4 n^{2}-1}-\frac{n e^{-n^{2} \beta \tau}}{2 \beta\left(4 n^{2}-9\right)}-\frac{n \tau e^{-n^{2} \beta \tau}}{4 n^{2}-1}\right)
\end{aligned}
$$

For more successive approximations it is more convenient to rewrite $(3.70)$ in the form

$$
a_{n}^{(K+1)}(\tau)=\frac{1}{\pi} e^{-n^{2} \beta \tau}+\frac{n}{2} \int_{0}^{\tau} e^{-n^{2} \beta(\tau-t)}\left[a_{n-1}^{(K)}(\tau)-a_{n+1}^{(K)}(\tau)\right] d t
$$

where the supercaript $K$ denotes the $k^{t h}$ approximation.

The form of higher order approximation is not given because it is extremely lengthy.

\section{$3.5 \quad$ RLC Ladder. Network Representation}

More insight into the system of equations (3.13), and hence to the analysis of the PLL, can be obtained crom a network theory point of view.

Equations (3.13) may be considered as the state equations of RLC ladder networks that have the Fourier coefficients as state variables.

This network representation of the problem provides a number of advantages, some are listed here:

a) Solution for the Fourter coefficients can be obtalned with the aid of a ladder network.

It is mentioned in the preceding section that the 
infinite system ( 3.13$)$ can be truncated, hence a solution can be obtained by applying one of the many techniques available in the literature for the analysis of a finite RLC ladder network [33]-[40].

By the virtue of this development the analysis of

ladder networks is directly applicable to the analysis of the PLI.

b) Stability study of the solution, for both finite and infinite systems, can be done via network theory. Stability of the finite system is discussed in sec. 3.5.2a and that of the infinite system in sec. 3.5.2b.

c) A study and interpretation of the transient process of the PLL, for different initial conditions and SNR, can be made in a laboratory using an RLC ladder network simulation.

\subsubsection{Realization of the RLC Ladder Networks}

This section gives the realization of the RLC ladder networks that have the Fourier coefficients, $a_{n}(\tau)$ and $b_{n}(\tau)$, as. state variables.

Consider the two infinite, coupled RLC ladder networks shown in Fig. 7 with state equations given by

$$
\begin{aligned}
& \dot{a}_{1}=-\frac{R_{1}}{L_{1}} a_{1}-\frac{1}{L_{1} a_{2}-\frac{r_{1}}{L_{1}} b_{1}+\frac{E}{L_{1}}} \\
& \dot{a}_{2}=\frac{1}{c_{2}} a_{1}-\frac{G_{2}}{c_{2}} a_{2}-\frac{1}{c_{2}} a_{3}-\frac{g_{2}}{c_{2}} b_{2}
\end{aligned}
$$

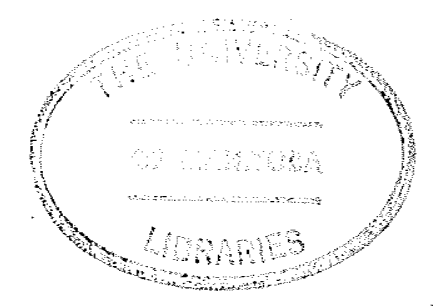



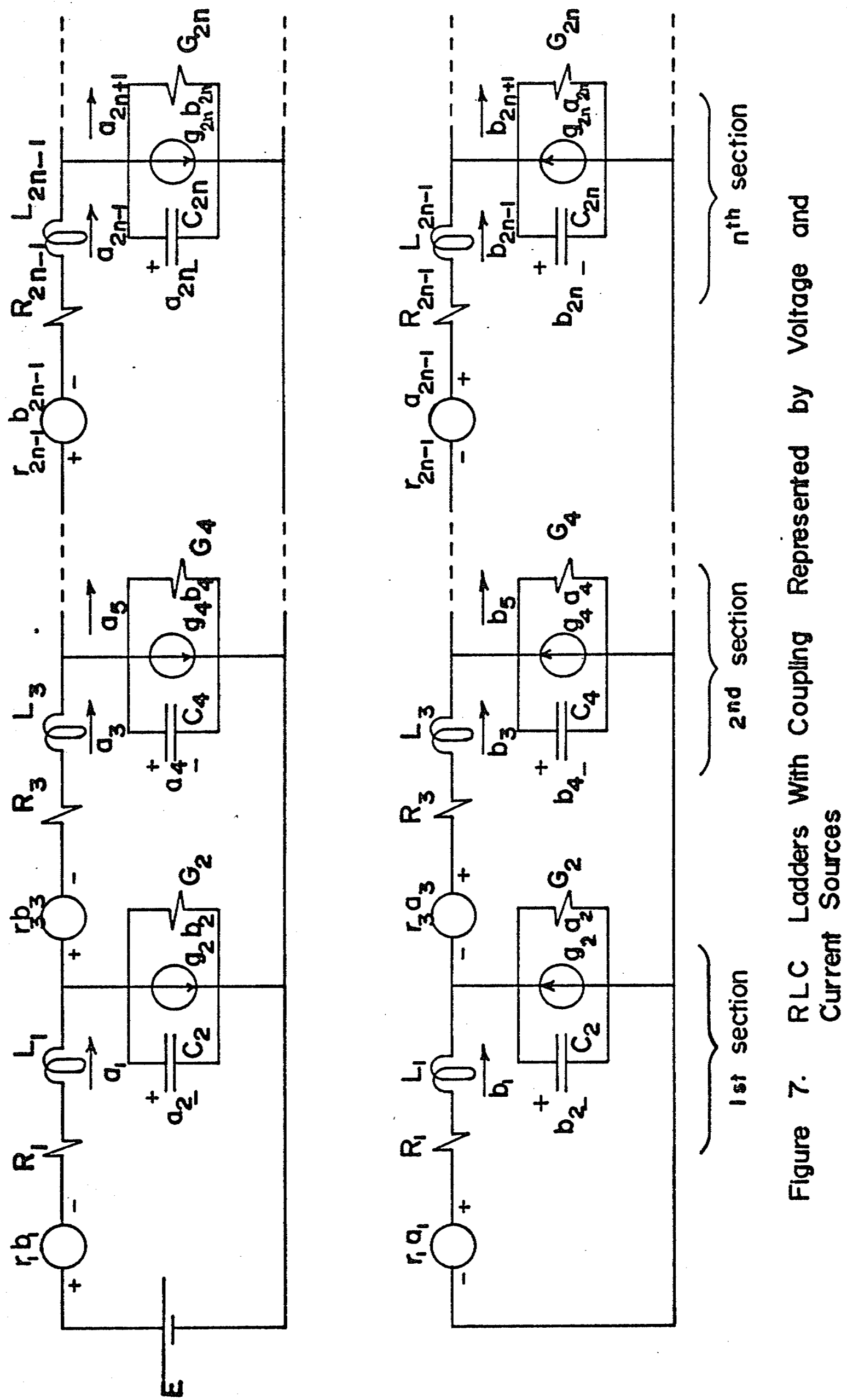


$$
\begin{aligned}
& \dot{a}_{3}=\frac{1}{L_{3}} a_{2}-\frac{R_{3}}{L_{3}} a_{3}-\frac{1}{L_{3}} a_{4}-\frac{r_{3}}{L_{3}} b_{3} \\
& \dot{a}_{n}=\frac{1}{L_{n}}\left(\text { or } \frac{1}{c_{n}}\right) a_{n-1}-\frac{R}{L_{n}}\left(\text { or } \frac{G_{n}}{c_{n}}\right) a_{n}-\frac{1}{L_{n}}\left(\text { or } \frac{1}{c_{n}}\right) a_{n+1}-\frac{r_{n}}{L_{n}}\left(\operatorname{or} \frac{g_{n}}{c_{n}}\right) b_{n} . \\
& \dot{b}_{1}=-\frac{R_{1}}{L_{1}} b_{1}-\frac{1}{L_{1}} b_{2}-\frac{r_{1}}{L_{1}} a_{1} \\
& \dot{b}_{2}=\frac{1}{c_{2}} b_{1}-\frac{G_{2}}{c_{2}} b_{2}-\frac{1}{c_{2}} b_{3}+\frac{g_{2}}{c_{2}} a_{2} \\
& \dot{b}_{3}=\frac{1}{L_{3}} b_{2}-\frac{R}{L_{3}} b_{3}-\frac{1}{L_{3}} b_{4}+\frac{r_{3}}{L_{3}} a_{3} \\
& \dot{b}_{n}=\frac{1}{L_{n}}\left(\text { or } \frac{1}{c_{n}}\right) b_{n-1}-\frac{R_{n}}{L_{n}}\left(\text { or } \frac{G_{n}}{c_{n}}\right) b_{n}-\frac{1}{L_{n}}\left(\text { or } \frac{1}{c_{n}}\right) b_{n+1}+\frac{r_{n}}{L_{n}}\left(\text { or } \frac{g_{n}}{c_{n}}\right) a_{n} \\
& \text { where } b=b(\tau) \text { and } a=a(\tau) \text {. }
\end{aligned}
$$

For the two systems (3.13) and ( 3.75$)$ to be identical, the elements of the ladder networks should have the values

$$
\begin{array}{ll}
R_{n}\left(\text { or } G_{n}\right)=2 \beta n & , \\
& L_{n}\left(\text { or } c_{n}\right)=\frac{2}{n}
\end{array}
$$




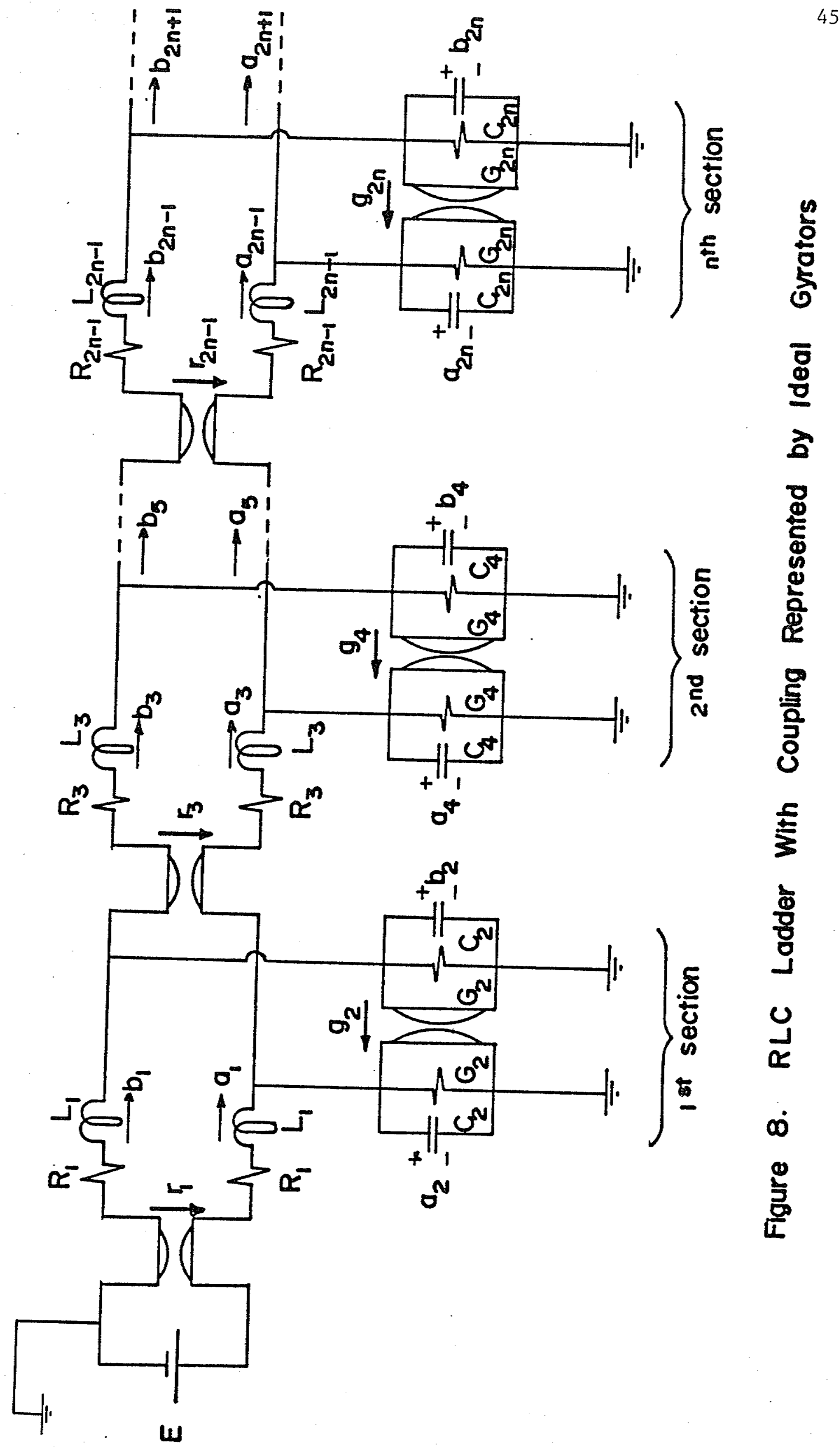


The two infinite ladder networks of Fig. 7 can be combined Into one network with the appropriate cholce for the coupling. It is found that the coupling elements can be ideal gyrators which are passive nonreciprocal two ports. The resulting network is shown in Fig. 8 .

\section{5 .2 Stability Study}

\subsection{2a Stability of the Finite RLC Ladder Network}

Since in the general case equations ( 3.13 ) have not been solved in closed form, it is expedient to resort to a numerical solution. In this case a finite number of sections of the RLC ladder network is used. Hence, a staility study of the finite system is necessary. This is carried out by applying the Liapunov method of stability to the ladder network of $n$ sections in Fig. 8 .

For zero input, the state equations can be rewritten

as

$$
\Lambda \dot{\mathrm{Z}}=-\mathrm{Hz}
$$

where

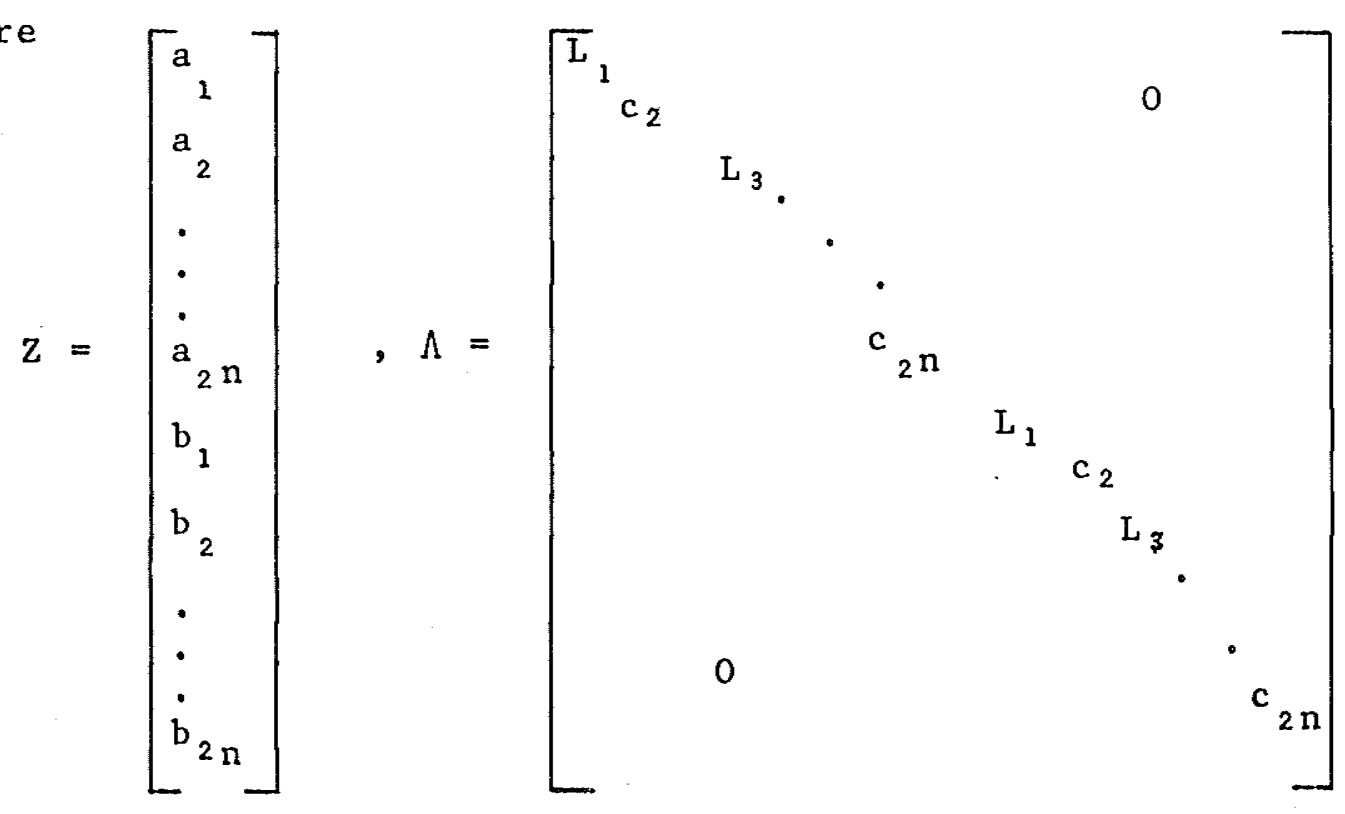




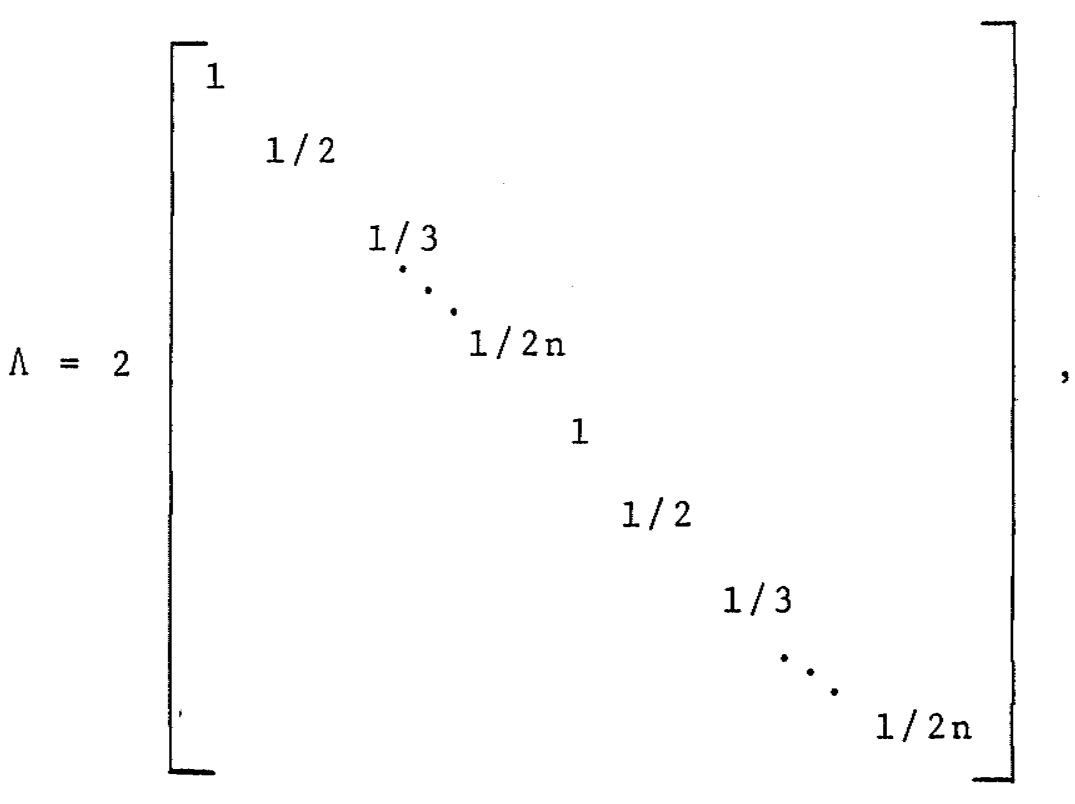

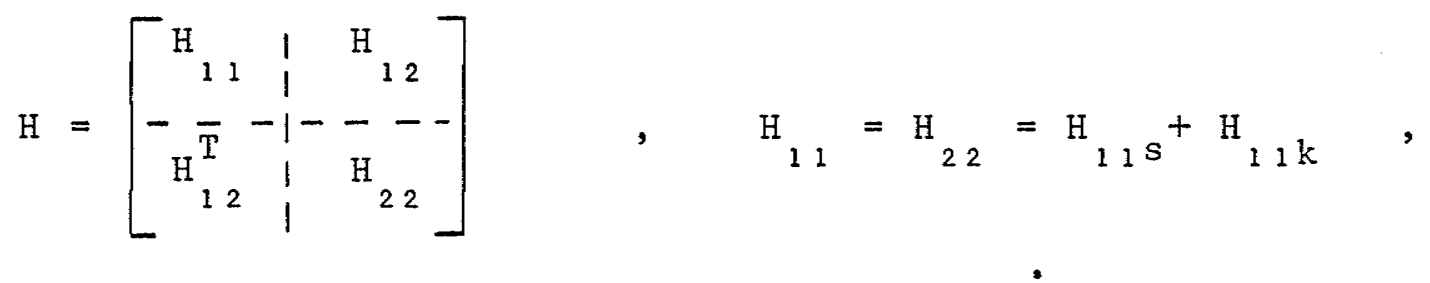

$\mathrm{H}_{1 \mathrm{is}} \triangleq$ the symmetric part of $\mathrm{H}_{11}$

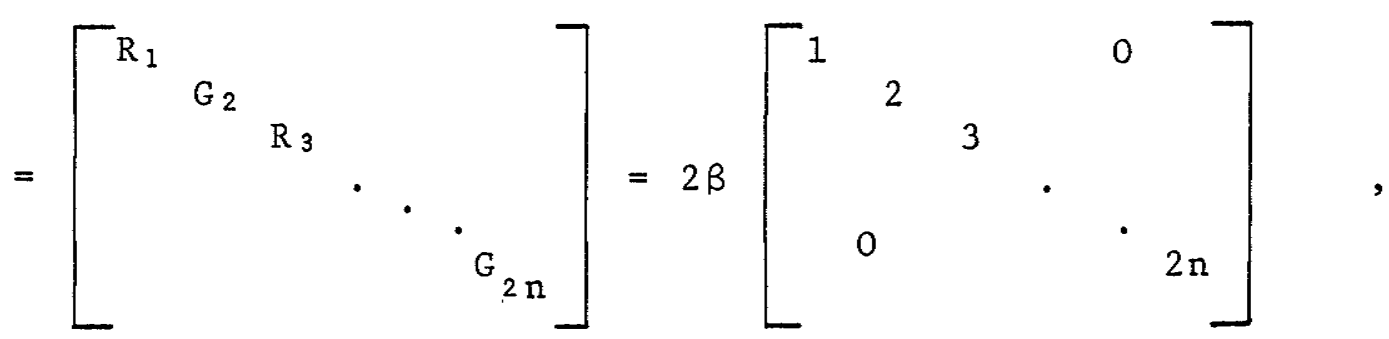

$H_{11 \mathrm{k}} \triangleq$ the skew symmetric part of $\mathrm{H}_{11}$

$$
=\left[\begin{array}{rrrrrrrr}
0 & 1 & & & & & \\
-1 & 0 & 1 & & & & \\
0 & -1 & 0 & 1 & & & \\
& & & \cdot & . & & & \\
& & & & -1 & 0 & 1 \\
& & & & & -1 & 0
\end{array}\right]
$$

and 


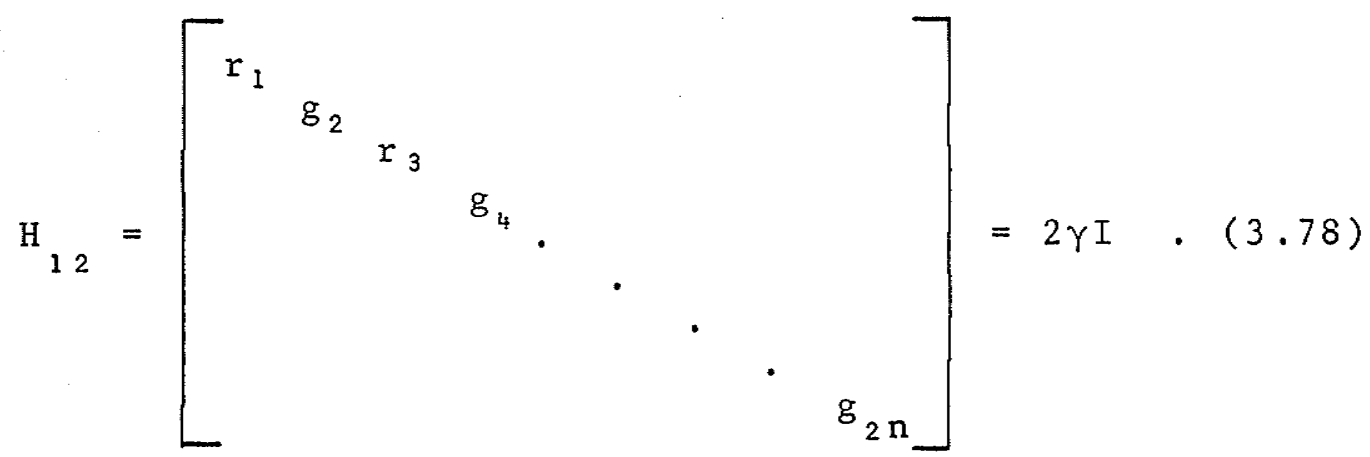

where $\mathrm{n}$ is the number of ladder sections.

Now let us pick the function

$\mathrm{V}(\mathrm{z}) \triangleq \frac{1}{2} \mathrm{z}^{\mathrm{T}} \Lambda \mathrm{z}>0 \quad \forall \mathrm{z} \neq 0$

as a tentative Liapunov function for the system ( 3.77 ). Then $\dot{V}(Z)$ along any trajectory is obtained as

$$
\begin{aligned}
\dot{\mathrm{V}}(\mathrm{z}) & =\frac{1}{2} \dot{\mathrm{z}}^{\mathrm{T}} \Lambda \mathrm{z}+\frac{1}{2} \mathrm{z}^{\mathrm{T}} \dot{\mathrm{z}} \\
& =-\frac{1}{2} \mathrm{z}^{\mathrm{T}} \mathrm{H}^{\mathrm{T}} \Lambda^{-1 \mathrm{~T}} \Lambda \mathrm{Z}-\frac{1}{2} \mathrm{Z}^{\mathrm{T}} \Lambda \Lambda^{-1} \mathrm{~Hz} \\
& =-\frac{1}{2} \mathrm{z}^{\mathrm{T}}\left(\mathrm{H}^{\mathrm{T}}+\mathrm{H}\right) \mathrm{z} \\
& =-\mathrm{z}^{\mathrm{T}} \mathrm{H}_{\mathrm{S}} \mathrm{P}
\end{aligned}
$$

where ${ }^{H}$ is the symmetric part of $\mathrm{H}$ given by

$$
\mathrm{H}_{\mathrm{S}}=\left[\begin{array}{ll}
\mathrm{H}_{11 \mathrm{~S}} & 0 \\
0 & \mathrm{H}_{11 \mathrm{~S}}
\end{array}\right]
$$




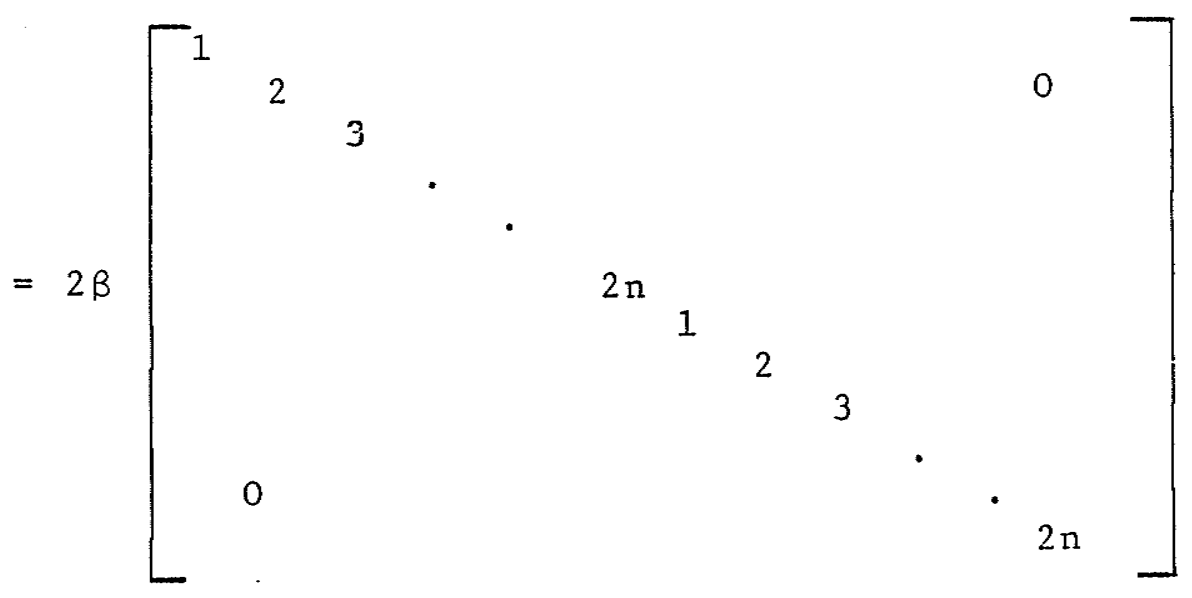

$(3.81)$

Introducing $(3.81)$ into $(3.80)$ yields, for finite values of B,

$$
\dot{V}(z)<0 \quad \forall z \neq 0
$$

Hence, the assumed function $V(Z)$ is a Liapunov function and the origin of the system ( 3.77$)$ is asymptotically stable in the large [22].

3.5.2b Stability Study of the Infinite Ladder

The stability of the infinite RLC ladder network of Fig. 8 can be studied by a comparison method. This method consists of comparing the ladder network of Fig. 8 to another one that has the same topology but different element values. The method is demonstrated here on the case when $\gamma=0$ and $\Phi_{0}=0$. In this case $b_{n}(\tau)=0$ and hence, the network of Fig. 8 will have the configuration of Fig. 9a. Consider the ladder network of Fig. $9 b$ with state variables, $C_{n}(\tau)$, satisfying the recurrence relation

$$
\dot{C}_{n}(\tau)=\frac{n}{2} c_{n-1}(\tau)-n \beta C_{n}(\tau)-\frac{n}{2} c_{n+1}(\tau) \quad, \quad n=1,2,3, \ldots
$$




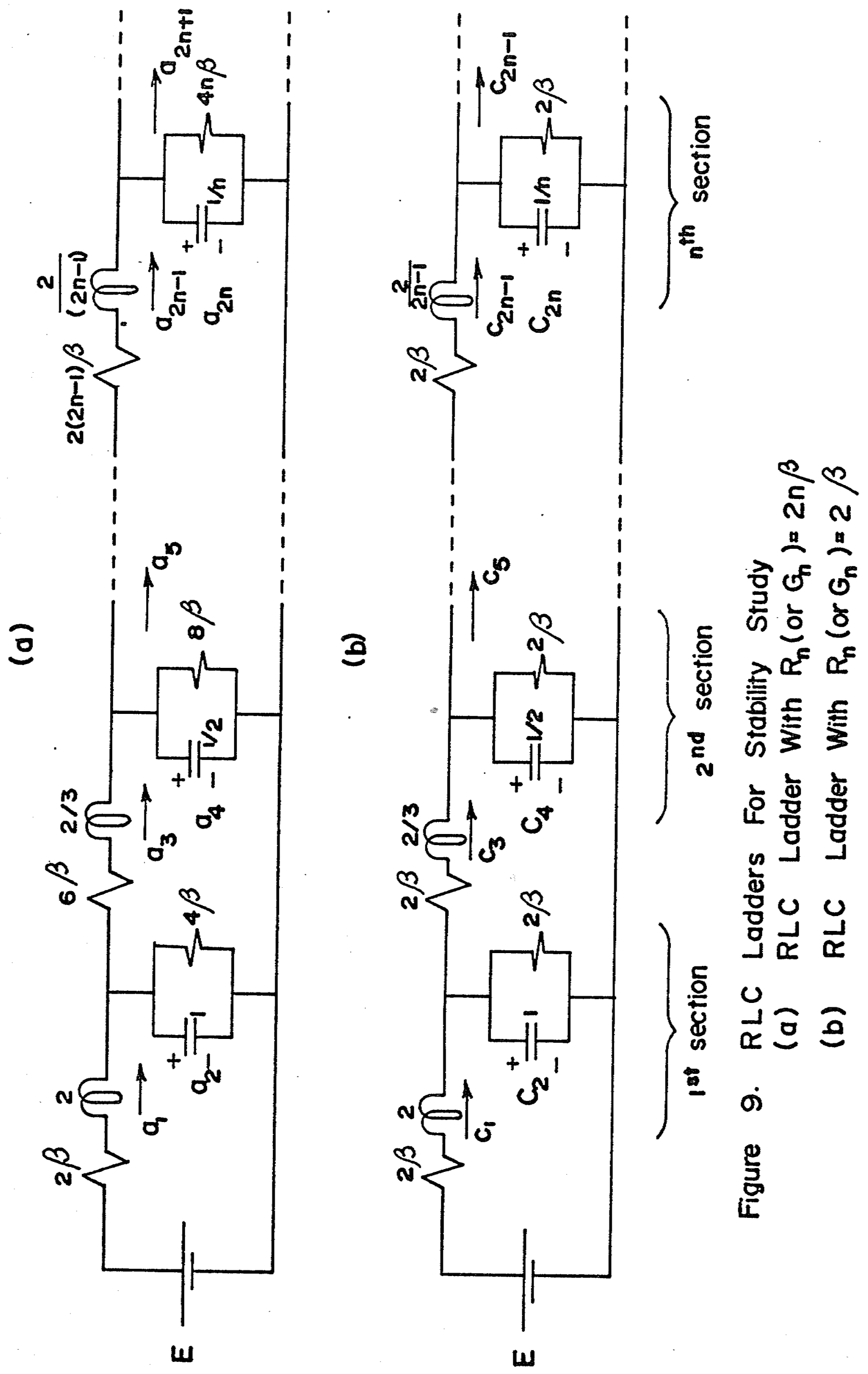


The resistances and conductances of the ladder network of Fig. 9a, except for the first resistance, are greater than those of Fig. 9b. Hence, the former ladder has more damping than the latter one. Assuming both networks have the same Initial conditions and the same input source, then from physical considexations (see Appendix I) it seems reasonable to assume that

$$
a_{n}(\tau) \leq c_{n}(\tau) \quad \text { for a } 11 \tau \quad \cdot \quad(3.84)
$$

Solutions of ( 3.83$)$, for both initial conditions discussed in Sec. 3.3, are given in Appendix C. The results are

$$
\begin{aligned}
& c_{n}(\tau)=\frac{1}{\pi}\left\{\sqrt{1+\beta^{2}} \tanh \left[\frac{\tau}{2} \sqrt{1+\beta^{2}}+\operatorname{arctanh}\left(\frac{\beta}{\sqrt{1+\beta^{2}}}\right)\right]-\beta\right\}^{n} \\
& \text { for } C_{n}(0)=a_{n}(0)=0
\end{aligned}
$$

and

$$
\begin{aligned}
& \quad C_{n}(\tau)=\frac{1}{\pi}\left\{a \frac{b \cosh \tau^{*}+a \sinh \tau^{*}}{a \cosh \tau^{*}+b \sinh \tau^{*}}-\beta\right\}^{n} \\
& \text { for } C_{n}(0)=a_{n}(0)=\frac{1}{\pi}
\end{aligned}
$$

where

$$
\begin{aligned}
& a=\sqrt{1+\beta^{2}} \\
& b=\beta+1 \\
& \tau^{*}=\frac{\tau}{2} \sqrt{1+\beta^{2}}
\end{aligned}
$$

From $(3.84),(3.85)$ and $(3.86)$ one can surmise that the infinite ladder of Fig. 9 a is stable. 


\subsection{Steady State Analysis}

The steady state modulo- $2 \pi$ probability density function and the variance of the phase error can also be obtained with the aid of the RLC ladder networks.

Since in the steady state $(\tau \rightarrow \infty)$ all the series inductors become short-circuited and all the shunt capacitors become open-circuited, the ladder network of Fig. 8 will have the configuration shown in Fig. 10 .

Applying Kirchhof's current and voltage laws yield,

$$
\begin{aligned}
& a_{n+1}=-2 n \beta a_{n}+a_{n-1}-2 \gamma b_{n} \\
& b_{n+1}=-2 n \beta b_{n}+b_{n-1}+2 \gamma a_{n} \quad n=1,2,3, \ldots(3.87)
\end{aligned}
$$

In the case of no coupling $(\gamma=0)$, it is evident that $b_{n}=0$ because there is no input source.

Hence, ( 3.87$)$ becomes

$$
a_{n+1}=-2 n \beta a_{n}+a_{n-1}
$$

and the corresponding network is shown in Fig. 11.

The recurrence formula ( 3.88$)$ is identical to that of the modified Bessel function [28] which is

$$
I_{n+1}\left(\frac{1}{\beta}\right)=-2 n \beta I_{n}\left(\frac{1}{\beta}\right)+I_{n-1}\left(\frac{1}{\beta}\right)
$$

Hence, a solution of $(3.88)$ can have the form

$$
a_{n}(\beta)=C(\beta) I_{n}\left(\frac{1}{\beta}\right)
$$




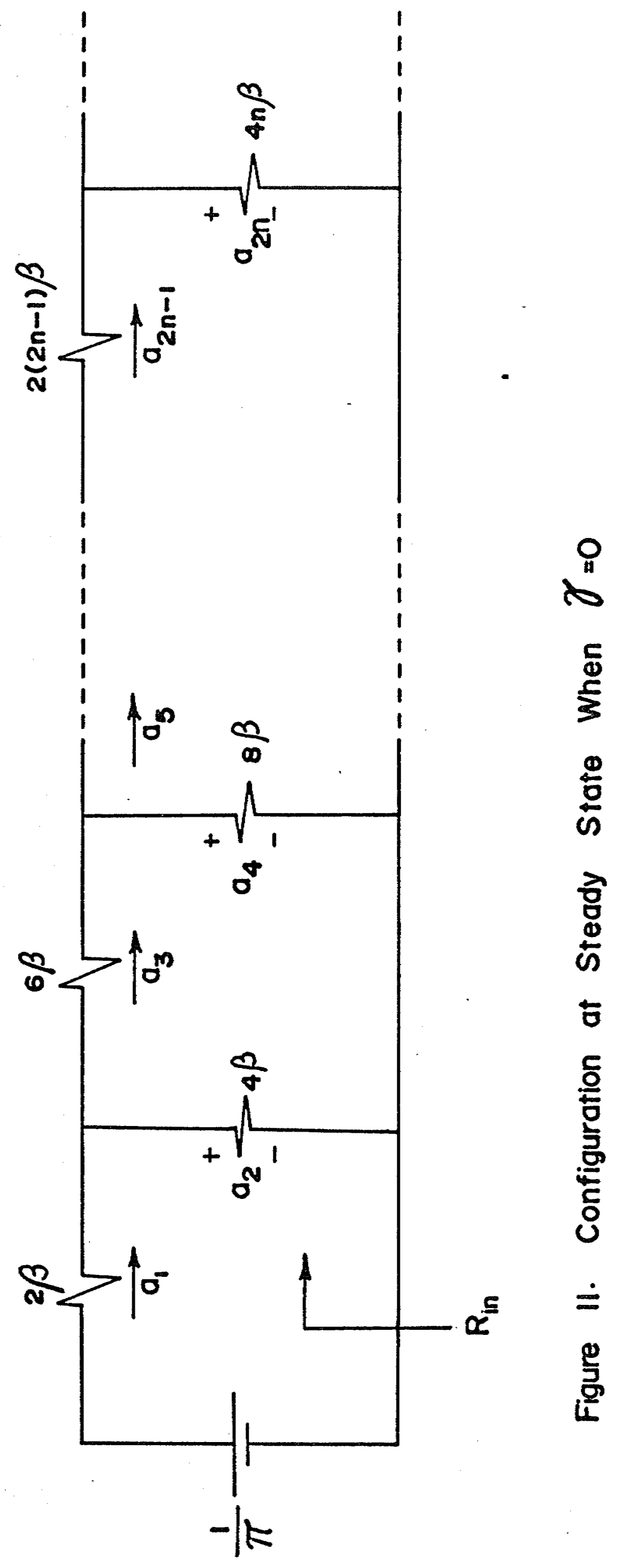


where $C(\beta)$ is a function of $\beta$ (NSR) and can be determined as follows.

The input resistance of the infinite resistive network of Fig. I1 is expressed by using the continued fraction

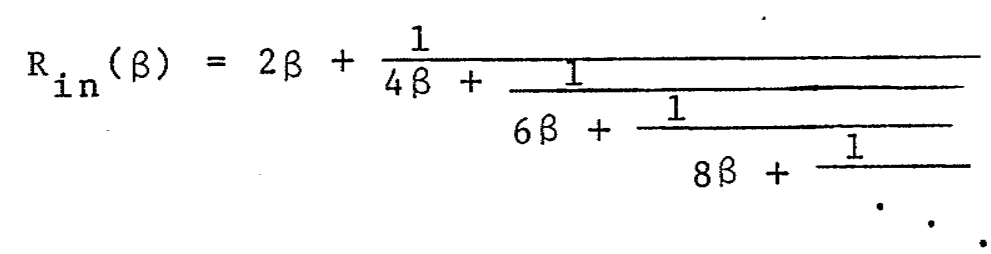

Using the series representation of the modified Bessel function of order $n$ [28], that is,

$$
I_{n}\left(\frac{1}{\beta}\right)=\left(\frac{1}{2 \beta}\right)^{n} \sum_{k=0}^{\infty} \frac{\left(\frac{1}{2 \beta}\right)^{2 k}}{k !(n+k) !}
$$

and by expressing the division $\frac{I_{0}\left(\frac{1}{\beta}\right)}{I_{1}\left(\frac{1}{\beta}\right)}$ in a continued fraction yields,

$$
\frac{I_{0}\left(\frac{1}{B}\right)}{I_{1}\left(\frac{1}{\beta}\right)}=2 \beta+\frac{1}{4 \beta+\frac{1}{6 \beta+\frac{1}{8 B+\frac{1}{.} .}}} .
$$

It is apparent from (3.91) and (3.93) that

$$
R_{\text {in }}(\beta)=\frac{I_{0}\left(\frac{1}{\beta}\right)}{I_{1}\left(\frac{1}{\beta}\right)}
$$

Hence

and

$$
a_{1}=\frac{E}{R_{\text {in }}}=\frac{I_{1}\left(\frac{1}{\beta}\right)}{\pi_{0}\left(\frac{1}{\beta}\right)}
$$

$$
C \quad(\beta)=\frac{1}{\pi I_{0}\left(\frac{1}{\beta}\right)}
$$

Introducing (3.95) into ( 3.90$)$ yields, 


$$
a_{n}=\frac{I_{n}\left(\frac{1}{\beta}\right)}{\pi I_{0}\left(\frac{1}{\beta}\right)}
$$

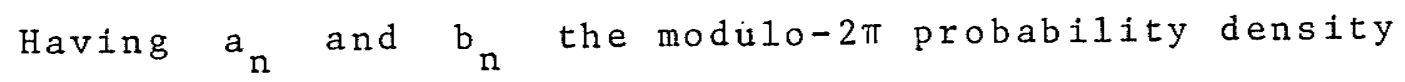
function and the variance of the phase error can be determined from $(3.6)$ and $(3.8)$ as

$$
\begin{aligned}
P(\Phi) & =\frac{1}{2 \pi}+\sum_{n \geq_{1}} \frac{I_{n}\left(\frac{1}{\beta}\right)}{\pi I_{0}\left(\frac{1}{\beta}\right)} \cos n \Phi \\
& =\frac{I}{2 \pi I_{0}\left(\frac{1}{\beta}\right)}\left[I_{0}\left(\frac{1}{\beta}\right)+2 \sum_{n \geq_{1}} I_{n}\left(\frac{1}{\beta}\right) \cos n \Phi\right] \\
& =\frac{1}{2 \pi I_{0}\left(\frac{1}{\beta}\right)} \cdot e^{\frac{1}{\beta} \cos \Phi}
\end{aligned}
$$

and

$$
\sigma_{\Phi}^{2}=\frac{\pi^{2}}{3}+4 \sum_{n \geq I} \frac{(-1)^{n} I_{n}\left(\frac{1}{\beta}\right)}{n^{2} I_{0}\left(\frac{1}{\beta}\right)} \text {. }
$$

The steady state modulo- $2 \pi$ phase error probability density function for the case when $\beta=0$ and $\gamma<1$ can be determined directly from the analytic solution given in sec. $4.3 .1 . b$. From $(3.58)$ and (3.59) one obtains

$$
\begin{aligned}
& f(\infty)=\arctan \frac{\gamma}{\sqrt{1-\gamma^{2}}}=\arcsin \gamma \\
& g(\infty)=1
\end{aligned}
$$

Introducing (3.99) and (3.100) into (3.52) yields,

$$
a_{n}(\infty)=\frac{1}{\pi} \cos [n(\arcsin \gamma)]
$$

and

$$
b_{n}(\infty)=\frac{1}{\pi} \sin [n(\arcsin \gamma)]
$$


Hence, from $(3.6)$ and $(3.101)$

$$
P(\Phi)=\frac{1}{2 \pi}+\frac{1}{\pi} \sum_{n=1}^{\infty} \cos n[\arcsin \gamma-\Phi] .
$$

Comparing $(3.102)$ and $(3.20)$, then

$$
P(\Phi)=\delta(\Phi-\arcsin \gamma)
$$

Hence, the steady state phase error

$$
\Phi_{0}=\arcsin \gamma
$$

It should be noticed that the steady state modulo- $2 \pi$ phase error probability density function and the variance, from equations (3.97) and (3.98), respectively, are consistent with those obtained by Viterbj. [3] . 
CHAPTER IV

TRANSIENT SHATSTIC.S OF THE PLL WITHOUT

RECOURSE TO THE FOKKER-PLANCK TECHNIQUE

\subsection{Introduction}

To obtain the phase error probability density function

for a PLL driven by a sinusoidal signal and a narrowband stationary noise process, the Fokker-Planck technique is usua11.y used.

Here another treatment is presented which deals directIy with the linearized stochastic differential equation of the loop phase error. It is shown [7], [8] and [29] that the solution of a linear stochastic differential equation, driven by a gatssian process, is a gauseian process with nean valie and variance satisfying certain ordinary differential equations of the first-order.

Applications of this theory to the first and secondorder PLL's are given.

4.2 First-order Phase-locked Loop

It has been shown in chapter II that the equation describes the first-order PLL operation, equation (2.15),

$$
\frac{d \phi(t)}{d t}=\left(\omega-\omega_{0}\right)-K\left[\operatorname{Asin} \phi(t)+n^{\prime}(t)\right] \quad \text {. (4.1) }
$$

Usirg the result of $[30]$, a linear system equivalent to (4.1) is found by determining a function $n(\alpha)$, a function 
of the loop signal-to-notse ratio, which minimizes the meansquare value of $E(\Phi)$ in the equation

$$
\frac{d \phi(t)}{d t}+\operatorname{KAn}(\alpha) \phi+\varepsilon(\phi)=\left(\omega-\omega_{0}\right)-K n^{\prime}(t)
$$

where

$$
\varepsilon(\phi)=\operatorname{AK}[\sin (\phi)-\eta(\alpha) \phi]
$$

The expected value of the mean-square of $\varepsilon(\phi)$ is

$$
E\left[\left(\varepsilon^{2}(\phi)\right]=A^{2} K^{2} \int_{-\infty}^{\infty}[\eta(\alpha) \phi-\sin \phi]^{2} p(\phi) d \phi\right.
$$

where $p(\phi)$ is the steady state phase error PDE.

The value of $\eta$ which minimizes the expression (4.4)

is

$$
\begin{aligned}
\eta(\alpha) & =\frac{\int_{-\infty}^{\infty} \phi \sin \phi p(\phi) d \phi}{\int_{-\infty}^{\infty} \phi^{2} p(\phi) d \phi} \\
& =\frac{\frac{\phi_{\text {sin }} \phi}{\sigma_{\phi}^{2}}}{\phi_{\text {s }}}
\end{aligned}
$$

where $\sigma_{\phi_{\mathrm{Ss}}}^{2}$ is the steady state phase error variance.

$$
\text { Now the equivalent linear system is found by dropping }
$$

the term $\varepsilon(\dot{\phi})$ in $(4,2)$. After a change of variables $(4.2)$

becomes

$$
\frac{\mathrm{d} \phi^{\prime}}{\mathrm{dt}}+\mathrm{KA} \eta \phi^{\prime}=-\eta \mathrm{K} \mathrm{n}^{\prime}
$$

where

$$
\phi^{\prime}=n \phi-\gamma \quad \text {, }
$$

and

$$
\gamma=\frac{\omega-\omega}{A K}
$$


Since $n^{\prime}$ is a gaussian process, the solution of the linear stochastic dfferential equation (4.6) is a gausian process with a mean value, $\mathrm{m}^{\prime}(\mathrm{t})$, and a variance, $\sigma_{\phi}^{2}(t)$, which satisfy the ordinary differential equations of the first-order [29]

$$
\frac{d m^{\prime}(t)}{d t}=-\operatorname{KAn} m^{\prime}(t)
$$

and

$$
\frac{d \sigma_{\phi^{\prime}}^{2}(t)}{d t}=-2 \mathrm{KAn} \sigma_{\phi^{\prime}}^{2}(t)+\eta^{2} K^{2} \frac{N_{0}}{2}
$$

where $\frac{N_{0}}{2}$ is the two-sided spectral density of $n^{\prime}$.

$$
\text { Solution of }(4.7) \text { and }(4.8) \text {, for } m^{\prime}(t) \text { and } \sigma_{\phi}^{2}(t)
$$
with the initial conditions $\mathrm{m}^{\prime}(0)=\eta_{\phi_{0}} \cdots \mathrm{y}^{\prime}$ and $\sigma_{\phi}^{2},(0)=0$, yields,

$$
m^{\prime}(t)=\left(\eta \phi_{0}-\gamma\right) e^{-\eta K A t}
$$

and

$$
\sigma_{\phi}^{2},(t)=\eta^{2} K^{2} \frac{N}{2}\left[1-e^{-2 K A \eta t}\right]
$$

Hence, the mean value, $m(t)$, and the variance, $\sigma_{\phi}^{2}(t)$, of $\phi$ will be

$$
m(t)=\frac{x}{n}+\left(\phi_{0}-\frac{x_{1}}{n}\right) e^{-n \tau} \quad .
$$

and

$$
\sigma_{\phi}^{2}(t)=\frac{1}{\alpha \eta}\left(1-e^{-2 \eta \tau}\right)
$$

where

$$
\tau=\mathrm{AKt},
$$

and

$$
\alpha=\frac{K N_{0}}{4 A}
$$


Now the value of $\eta(\alpha)$ can be determined as follows. Since one is usually interested in $\phi$ for the range $(-\pi, \pi)$, a straightforward adjustment of (4.5) results in an equation that is appropriate for $|\Phi| \leq \pi$. The result is

$$
\eta(\alpha)=\frac{\int_{-\pi}^{\pi} \Phi \sin \Phi P(\Phi) d \Phi}{\int_{-\pi}^{\pi} \Phi^{2} \cdot P(\Phi) d \Phi}
$$

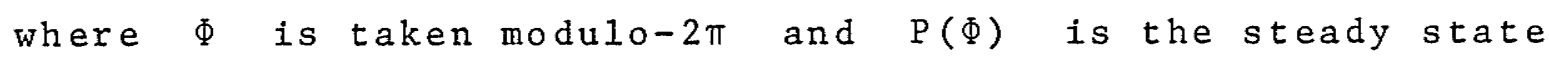
modu $10-2 \pi$ phase error PDF.

In the case $\gamma=0, P(\Phi)$ and $\sigma_{\Phi}^{2}$ will have the values given in $(3.97)$ and (3.98), respectively. Introducing these values into (4.13) and integrating yields, upon letting $\beta=\frac{1}{\alpha}$,

$$
n(\alpha)=\frac{\frac{1}{\alpha}\left[I_{0}(\alpha)-e^{-\alpha}\right]}{\frac{\pi^{2}}{3} I_{0}(\alpha)+4 \sum_{n \geq 1} \frac{(-1)^{n} I_{n}(\alpha)}{n^{2}}} \quad \text { (4.14) }
$$

This series converges so rapidly that ( 4.14 ) can be calculated with considerable accuracy by using only a few terms of the expansion [28].

Hence, the modulo- $2 \pi$ phase error PDF of the linearized first-order PLL is

$$
P(\Phi, \tau)=\sqrt{\frac{\alpha \eta}{2 \pi}\left(1-e^{-2 \eta \tau}\right)}
$$$$
\begin{aligned}
& e^{-\frac{\alpha \eta\left(\Phi-\Phi_{0} e^{-\eta \tau}\right)^{2}}{2\left(1-e^{-2 \eta \tau}\right)}} \\
& |\Phi| \leq \pi \quad,(4.15)
\end{aligned}
$$ 
4.3 Second-order Phase-locked Loop

In order to analyze the second-order PLL, it should be realized that the main difference from the first-order PLL is the loop filter transfer function. In this case the loop filter transfer function is in the form

$$
F(s)=\frac{G(s)}{H(s)}=\frac{1+\tau_{1} s}{1+\tau_{2} s},
$$

The loop filter of greatest interest is

$$
F(s)=\frac{s+a}{s}
$$

which requires a single integrator with gain a.

The equation that describes the operation of the second-order PLI, whose filter transfer function is given by $(4.17)$, is, from $(2.6)$,

$$
\begin{aligned}
\frac{d \phi(t)}{d t}=\left(\omega-\omega_{0}\right) & -K\left[\operatorname{Asin} \phi(t)+n^{\prime}(t)\right] \\
& -a K \int_{0}^{t}\left[\operatorname{Asin} \phi(u)+n^{\prime}(u)\right] d u,(4.18)
\end{aligned}
$$

Equation ( 4.18 ) cannot be differentiated further, since this would result in an equation involving the derivative of white noise.

It is clear from ( 4.18$)$ that the process generated by driving a second-order PLL with white gausian noise is not a Markov process, however the output of the second-order PLL driven by white gausian noise can be decomposed into two first-order equations in the process and its first derivative to constitute a two-dimensional vector Markov process [3]. Now if the substitution 


$$
\phi(t)=x_{2}(t)+a x_{1}(t),
$$

where $\quad x_{2}(t)=\frac{d x_{1}(t)}{d t}$, is made in (4.18) one obtains [3] the two first-order differential equations

$$
\dot{x}_{1}(t)=x_{2}(t)
$$

and

$$
\dot{x}_{2}(t)=-\operatorname{KAsin}\left[\mathrm{ax}_{1}(t)+\mathrm{x}_{2}(t)\right]-K n^{\prime}(t) \text {. }
$$

The second equation of $(4.20)$ can be linearized by letting $\sin \phi \approx \phi$, and hence, (4.20) can be written in the form

$$
\frac{d x}{d t}=B x+e
$$

where

$$
x=\left[\begin{array}{c}
x_{1} \\
x_{2}
\end{array}\right], \quad B=\left[\begin{array}{cc}
0 & 1 \\
-K A a & -K A
\end{array}\right] \text { and } e=\left[\begin{array}{c}
0 \\
-K_{n}^{\prime} \\
(4.20)
\end{array}\right]_{(420)}
$$

Since $n^{\prime}(t)$ is a stationary gausian process, it seems reasonable to model the loop operation by the following linear stochastic differential equation [29]

$$
d x=B x d t+d v
$$

where $v(t)$ is a two-dimensional Weiner process with variance parameter $\frac{\mathrm{N}_{0} \mathrm{~K}^{2}}{2}$.

By applying the theory of Aström [29], the solution of (4.23) is a gaussian process with mean value, $m(t)$, and variance, $V(t)$, satisfying the following ordinary differential equations of the first-order 


$$
\frac{d m(t)}{d t}=B m(t)
$$

and

$$
\frac{d V(t)}{d t}=B V+V B^{T}+R_{1}
$$

where

$$
m(t)=\left[\begin{array}{l}
m_{1}(t) \\
m_{2}(t)
\end{array}\right], \quad V(t)=\left[\begin{array}{cc}
v_{1}(t) & v_{2}(t) \\
v_{2}(t) & v_{3}(t)
\end{array}\right] \text {, }
$$

and

$$
R_{1}=\left[\begin{array}{cc}
0 & 0 \\
0 & \frac{\mathrm{N}_{0} \mathrm{k}^{2}}{2}
\end{array}\right]
$$

Solution of $(4.24)$ and $(4.25)$, with the appropriate initial conditions, is given in Appendix G; the results are

$$
\begin{aligned}
& V_{1}(t)=\frac{N_{0}}{4 A^{2} a}\left[1-e^{-A K t}\left(1+\frac{A^{2} K^{2}}{2} \frac{\sin ^{2} \Omega t}{\Omega^{2}}+A K \frac{\sin 2 \Omega t}{2 \Omega}\right)\right],(4.27) \\
& V_{2}(t)=\frac{N_{0} K^{2}}{4} e^{-A K t} \frac{\sin ^{2} \Omega t}{\Omega^{2}} \\
& V_{3}(t)=\frac{N_{0} K^{2}}{4 A}\left[1-e^{-A K t}\left(1+\frac{A^{2} K^{2}}{2} \frac{\sin ^{2} \Omega t}{\Omega^{2}}-A K \frac{\sin 2 \Omega t}{2 \Omega}\right)\right], \\
& m_{1}(t)=e^{-A K t / 2\left[\frac{\sin \Omega t}{\Omega} x_{2}(0)+\left(\cos \Omega t+\frac{A K}{2} \frac{\sin \Omega t}{\Omega}\right) x_{1}(0)\right]}
\end{aligned}
$$

and

$$
m_{2}(t)=e^{-A K t / 2}\left[\left(\cos \Omega t-\frac{A K}{2} \frac{\sin \Omega t}{\Omega}\right) x_{2}(0)-\left(A K a \frac{\sin \Omega t}{\Omega}\right) x_{1}(0)\right]
$$

where

$$
\Omega^{2}=A K a-\frac{A^{2} K^{2}}{4}
$$


Now, since $\phi$ is related linearly to $x_{1}$ and $x_{2}$, as $\phi=\mathrm{ax}_{1}+\mathrm{x}_{2}$, the conditional probability density function $\mathrm{p}\left(\phi \mid \phi_{0}, t\right)$ will be gaussian. One obtains from $(4.27)-(4.31)$ for the average value and the variance, the expressions:

$$
\begin{aligned}
\bar{\phi} & =x_{2}(0) e^{-A K t / 2}\left[\left(a-\frac{A K}{2}\right) \frac{\sin \Omega t}{\Omega}+\cos \Omega t\right] \\
& +x_{1}(0) e^{-A K t / 2}\left[a \cos \Omega t-\frac{A K a}{2} \frac{\sin \Omega t}{\Omega}\right]
\end{aligned}
$$

and

$$
\begin{aligned}
\overline{(\phi-\bar{\phi})^{2}} & =\frac{\mathrm{N}}{4 \mathrm{~A}}\left[\frac{\mathrm{a}}{\mathrm{A}}+\mathrm{K}\right)\left[1-\mathrm{e}^{-\mathrm{AKt}}\left(1+\frac{\mathrm{A}^{2} \mathrm{~K}^{2}}{2} \frac{\sin ^{2} \Omega \mathrm{t}}{\Omega^{2}}\right)\right] \\
& +\mathrm{e}^{-\mathrm{AKt} \frac{\mathrm{N}_{0} \mathrm{~K}}{4} \frac{\sin 2 \Omega \mathrm{t}}{2 \Omega}\left(\mathrm{K}-\frac{\mathrm{a}}{\mathrm{A}}\right)} \\
& +\frac{\mathrm{aK}^{2} \mathrm{~N} 0}{2} \mathrm{e}^{-\mathrm{AKt}} \frac{\sin ^{2} \Omega \mathrm{t}}{\Omega^{2}}
\end{aligned}
$$

Since the average value and the variance are now known the gausian process is completely characterized.

Because of the cycle skipping phenomena (due to the nonlinearity and the gausian noise), the phase error variance in the steady state is infinite. However, in the linearized model the steady state phase error variance has a finite value. By letting $t \rightarrow \infty$ in (4.33) yields,

$$
\sigma_{\phi_{S S}}^{2}=\overline{(\phi-\bar{\phi})^{2}}=\frac{\mathrm{N}_{0}}{4 \mathrm{~A}}\left(\frac{\mathrm{a}}{\mathrm{A}}+\mathrm{K}\right)
$$




\section{CHAPTER V}

\section{NUMERICAL RESULTS}

This chapter contains the results of the simulation of the finite RLC ladder networks shown in F1g. 7. Also, in part two of this chapter, the results of the linearization technique, used in Sec. 4.2 and the approximation $\sin \Phi \approx \Phi$ are presented.

\subsection{Truncated Ladder Technique}

The simulation of a finite number of sections (20) of the RLC ladder networks, shown in Fig. 7, was carried out on an IBM-360 computer using the Continuous system Modeling Program ( $\left.\operatorname{CSMP} 30^{\circ} 0\right)$.

Tables I and II indicate the various cases which were studied for initial conditions $P(\Phi, 0)=\delta\left(\Phi-\Phi{ }_{0}\right)$ and $P(\Phi, 0)=\frac{1}{2 \pi}$, respectively. The specific values vere chosen so as to provide comparison with previously published work $[3],[10],[13],[14]$ and $[15]$.

It is found that the state variables, $a_{n}(\tau)$ and $b_{n}(\tau)$, decrease rapidly as $n$ increase, for fixed . On this bases, for the calculation of $P(\Phi, \tau)$ a finite number of variables $2 n$ where $n<20$ is taken. This $n$ is chosen such thst the value of the $(2 n+1)$ state variable is of the order of $10^{-6}$ of the first state variable. This is shown together with the corresponding $n$ in the last and second columns (of both tables), respectively. It is 
interesting to note that the number of sections increases as the signal-to-noise ratio $(\alpha)$ increases.

The transitional modulo- $2 \pi$ density is depicted in Figs. 12-17 and in Figs. 19-21 for the cases shown in Tables I and II, respectively.

Figs. 18 and 22 are plots of the phase error variance for the two initial conditions $P(\Phi, 0)=\delta(\Phi)$ and $P(\Phi, 0)=$ $\frac{1}{2 \pi}$, respectively.

Figs. 13 and 16 are essentially identical to the curves obtained by ohlson and Rutherford [14] in their numerical technique. However, the computation time required to produce Figs. 13 and 16 is much smaller than the time required by the algorithm in [14].

Fig. 17 shows the modulo-2 $2 \pi$ density for various time, $\tau$. This data agrees with that obtained by La Frieda $[10]$

The steady state modulo- $2 \pi$ PDF determined numericalIy in this analysis agrees, for $\gamma=0$, with the analytical results found by the method in Sec. 3.6 and with viterbi's results [3]. The results obtained for a detuned loop as shown in Figs. 16 and 20 agree with that obtained by Viterbi [3]. As is expected, these plots demonstrate that the detuned loop exhibits a definite tendency to slip in the direction of the steady state phase shift caused by detuning.

The set of conditions in Fig. 14 are the same as one of those selected by Dominiak and Pickholtz [13] in their numerical analysis of the first-order PLL. The values of 
$P(\Phi, \tau)$, for small $\tau$, in Fig. 14 are different from those in [13]. This is expected because the technique used in [13] is not capable of accurately determining the time reference. Thus, the times appearing in their curves are different from the actual times and therefore, their plot does not truly represent the dependence of $P(\Phi, \tau)$ on time. The phase error variance is shown in Figs. 18 and 22 as a function of time for different values of SNR $(\alpha=2.2,2.8,3.5$ and 5.0$)$. The set of conditions of Fig. 18 are the same as those selected by Grandoni and Mengali [15] in their analysis. Their curves, identified by asterisks, together with those obtained by this study are shown in Fig. 18. The difference in the results, especially for small $\alpha$, is due to the fact that they assumed a gaussian form for the PDF. However, this difference in the results, as it is seen in Fig. 18, diminishes as $\alpha$ increases.

Figs. 18 and 22 also illustrate that the transient time decreases as $\alpha$ increases. This can be easily explained by noting that a large $\alpha$ implies less damping in the RLC structure and therefore, the transient time is smaller.

\subsection{Einearization Methods}

In this section the results of the linearization technique mentioned in sec. 4.2 are presented. Also, the results of the approximate solution, in which $\sin \Phi \approx \Phi$, are plotted. 
In Figs. 23-26 the PDF of the linearized 10op (curves labeled $\eta \neq 1$ ) is compared with the PDF obtained by the truncated RLC ladder technique (curves labeled exact), for various cases.

In Figs. 27-29 the results of the approximation $\sin \Phi \approx \Phi$ (curves labeled $\eta=1$ ) are compared with those of the linearization technique and the exact solution obtained by the truncated RLC ladder method.

It can be seen that, for small values of $\alpha$, the Iinearization method works better than the approximation $\sin \Phi \approx \Phi$ if $|\Phi| \leq \pi$. 
TABLE I

$P(\Phi, 0)=\delta\left(\Phi-\Phi_{0}\right)$

\begin{tabular}{|c|c|c|c|c|c|}
\hline$\alpha$ & $\mathrm{n}$ & $\Phi_{0}$ & $\gamma$ & $\tau$ & $\begin{array}{l}\text { The }(2 n+1) \text { state } \\
\text { variable }\end{array}$ \\
\hline 0.1 & 2 & 0 & 0 & $\begin{array}{c}0.06 \\
0.15 \\
\infty\end{array}$ & $\begin{array}{l}1.45 \times 10^{-6} \\
1.12 \times 10^{-8} \\
8.27 \times 10^{-10}\end{array}$ \\
\hline \multirow[t]{2}{*}{1.0} & \multirow[t]{2}{*}{6} & \multirow[t]{2}{*}{0} & 0 & $\begin{array}{l}0.06 \\
0.24 \\
0.51 \\
0.8 \\
1.0 \\
\infty\end{array}$ & $\begin{array}{l}5.79 \times 10^{-6} \\
6.27 \times 10^{-12} \\
5.36 \times 10^{-24} \\
1.51 \times 10^{-14} \\
1.05 \times 10^{-14} \\
5.05 \times 10^{-15}\end{array}$ \\
\hline & & & $\sin \frac{\pi}{4}$ & $\begin{array}{l}0.06 \\
0.51 \\
1.0 \\
\infty\end{array}$ & $\begin{array}{l}4.93 \times 10^{-6} \\
3.38 \times 10^{-15} \\
2.9 \times 10^{-15} \\
1.68 \times 10^{-15}\end{array}$ \\
\hline 2.0 & 9 & $\frac{\pi}{2}$ & 0 & $\begin{array}{l}0.06 \\
0.50 \\
1.0 \\
5.0\end{array}$ & $\begin{array}{l}1.96 \times 10^{-6} \\
2.47 \times 10^{-17} \\
1.37 \times 10^{-18} \\
1.18 \times 10^{-18}\end{array}$ \\
\hline 5.0 & 10 & 0 & 0 & $\begin{array}{l}0.15 \\
1.0 \\
\infty\end{array}$ & $\begin{array}{l}1.32 \times 10^{-6} \\
2.4 \times 10^{-12} \\
6.04 \times 10^{-13}\end{array}$ \\
\hline
\end{tabular}


TABLE II

$P(\Phi, 0)=\frac{1}{2 \pi}$

\begin{tabular}{|c|c|c|c|c|}
\hline$\alpha$ & $n$ & $\gamma$ & $\tau$ & $\begin{array}{l}\text { The }(2 n+1) \text { state } \\
\text { variable }\end{array}$ \\
\hline \multirow{2}{*}{1.0} & \multirow{2}{*}{6} & 0 & $\begin{array}{l}.0 \\
0.15 \\
0.51 \\
i .0 \\
\infty\end{array}$ & $\begin{array}{l}0 \\
6.77 \times 10^{-19} \\
4 \times 10^{-16} \\
1.96 \times 10^{-15} \\
5.05 \times 10^{-15}\end{array}$ \\
\hline & & $\sin \frac{\pi}{4}$ & $\begin{array}{c}0 \\
0.15 \\
0.51 \\
1.0 \\
\infty\end{array}$ & $\begin{array}{l}0 \\
3.32 \times 10^{-19} \\
3.51 \times 10^{-16} \\
1.7 \times 10^{-16} \\
1.69 \times 10^{-9}\end{array}$ \\
\hline 5.0 & 10 & 0 & $\begin{array}{c}0 \\
0.15 \\
0.51 \\
1.0 \\
3.55\end{array}$ & $\begin{array}{c}0 \\
2.19 \times 10^{-25} \\
5.91 \times 10^{-18} \\
2.64 \times 10^{-15} \\
6.04 \times 10^{-13}\end{array}$ \\
\hline
\end{tabular}




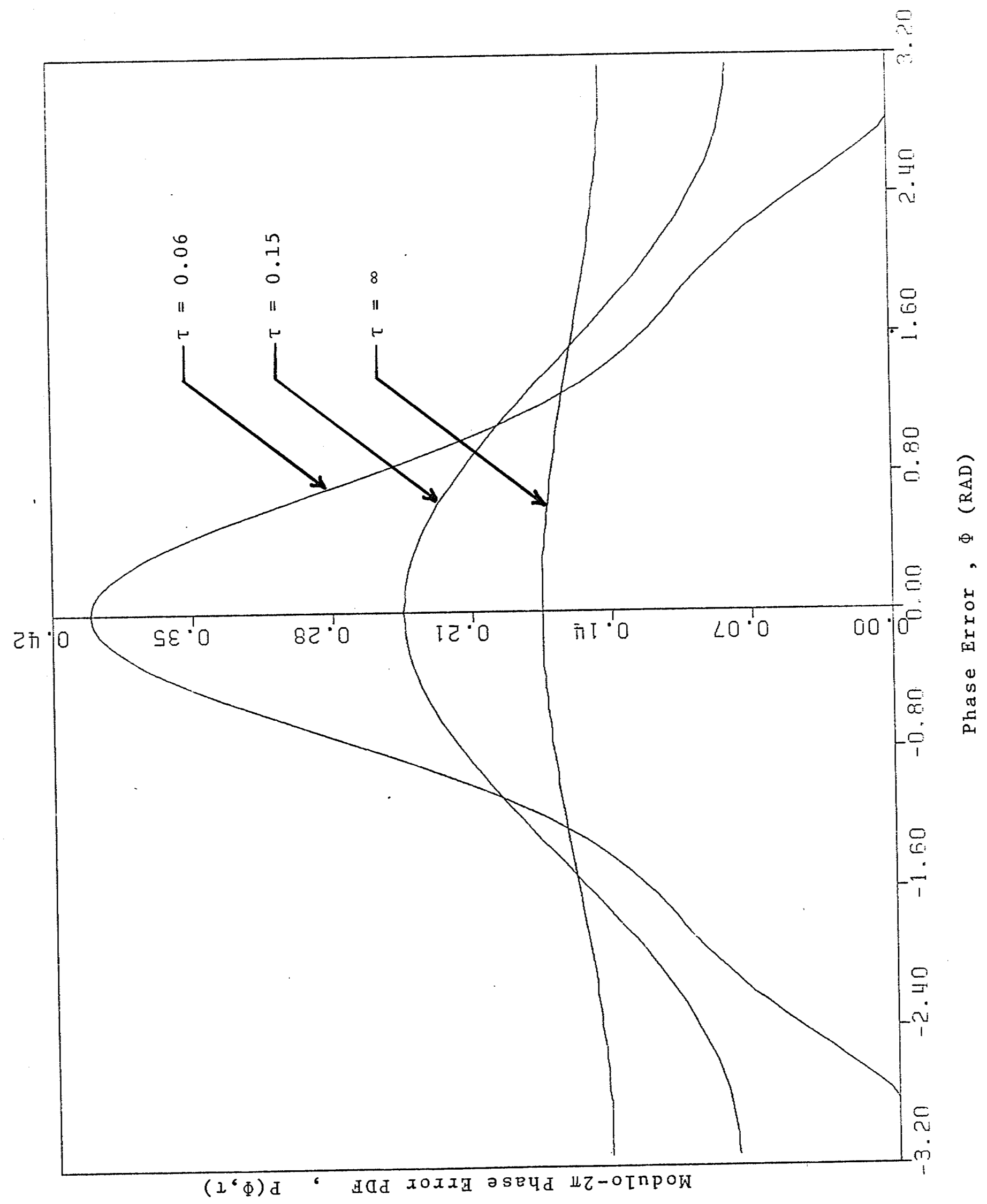

Figure 12. Modulo-2 $2 \pi$ Phase Error Probability Density Function $\alpha=0.1, \Phi_{0}=0, \quad \gamma=0$. 


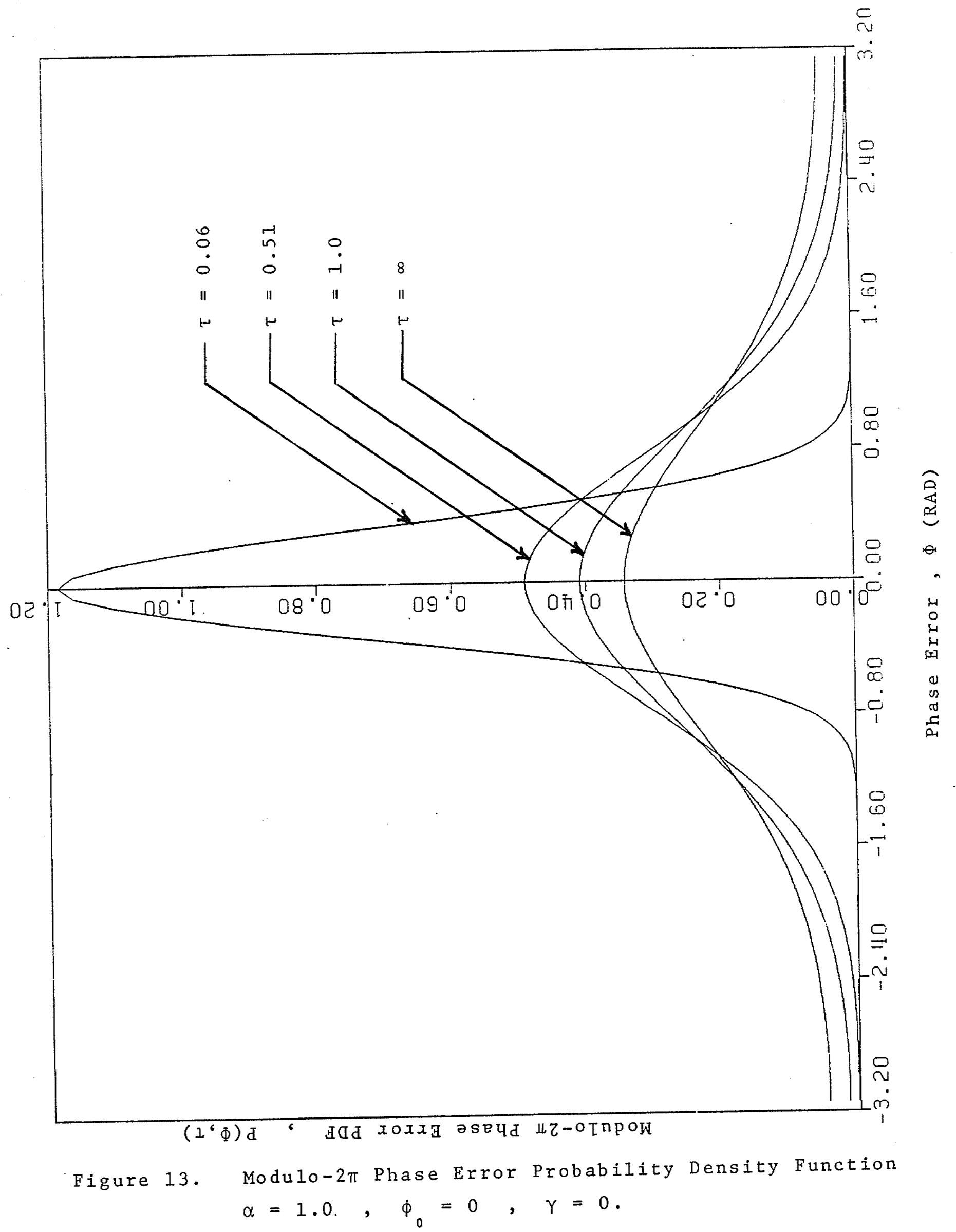




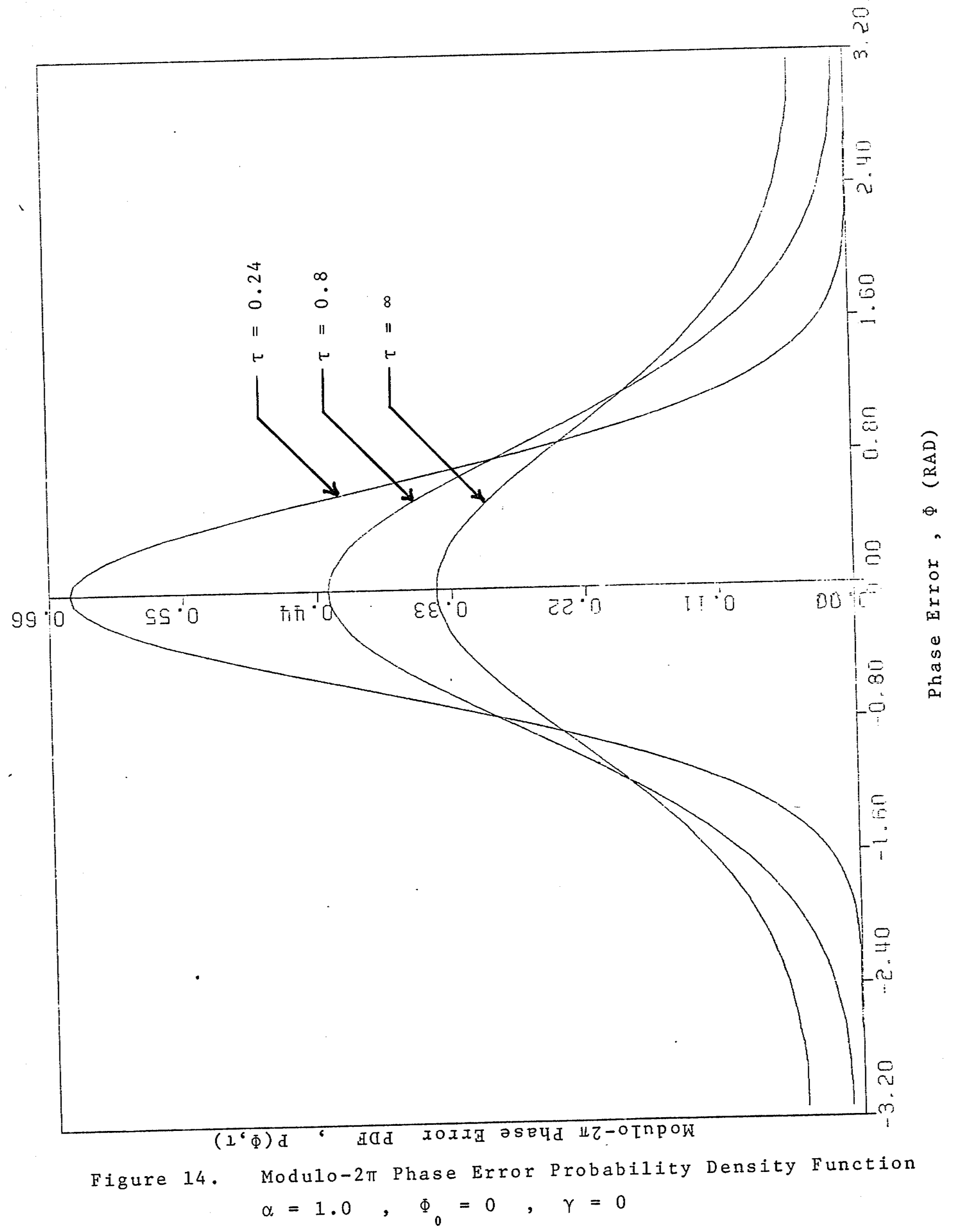




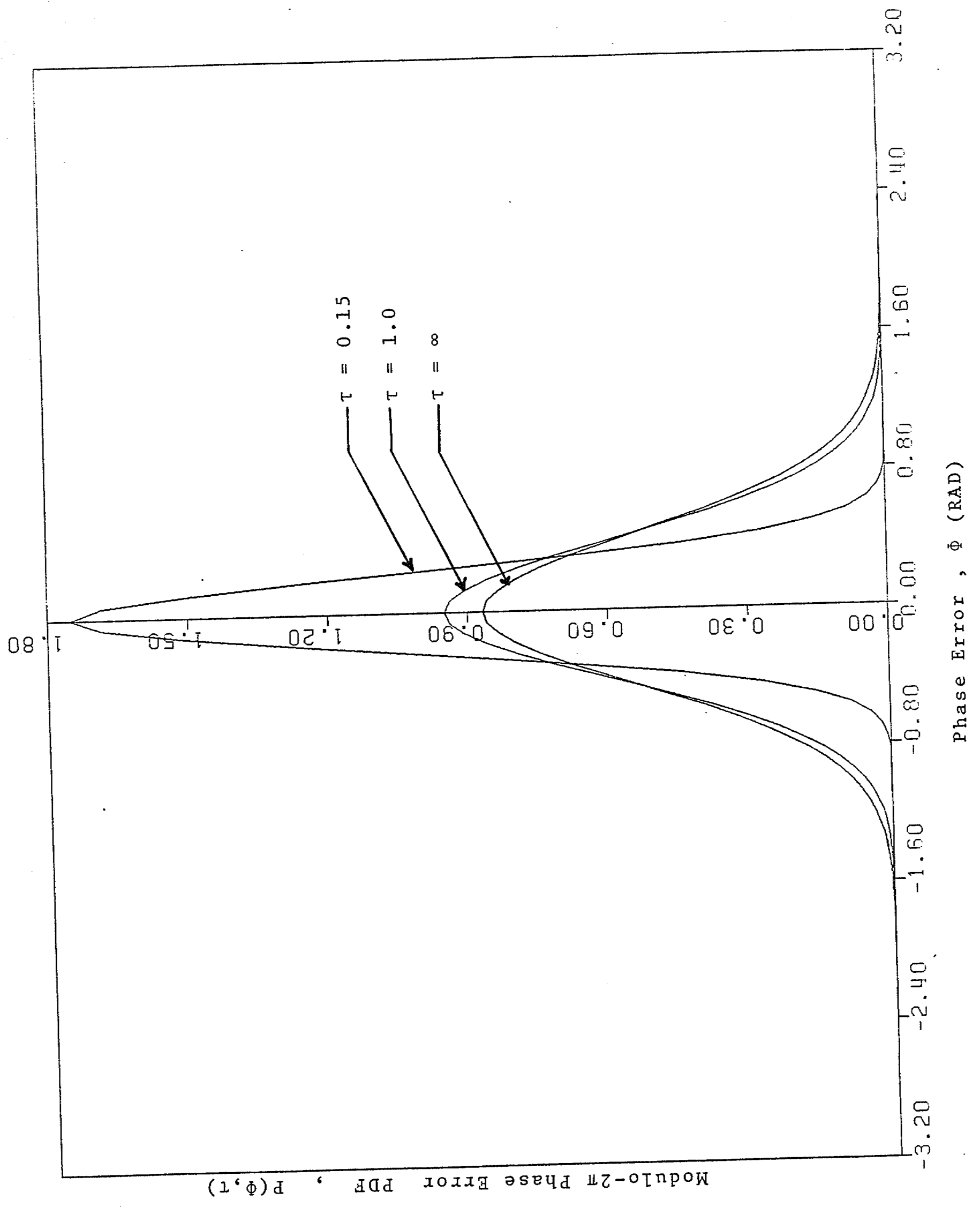

Figure 15. Modulo-2 2 Phase Error Probability Density Function $\alpha=5.0, \Phi_{0}=0, \gamma=0$. 


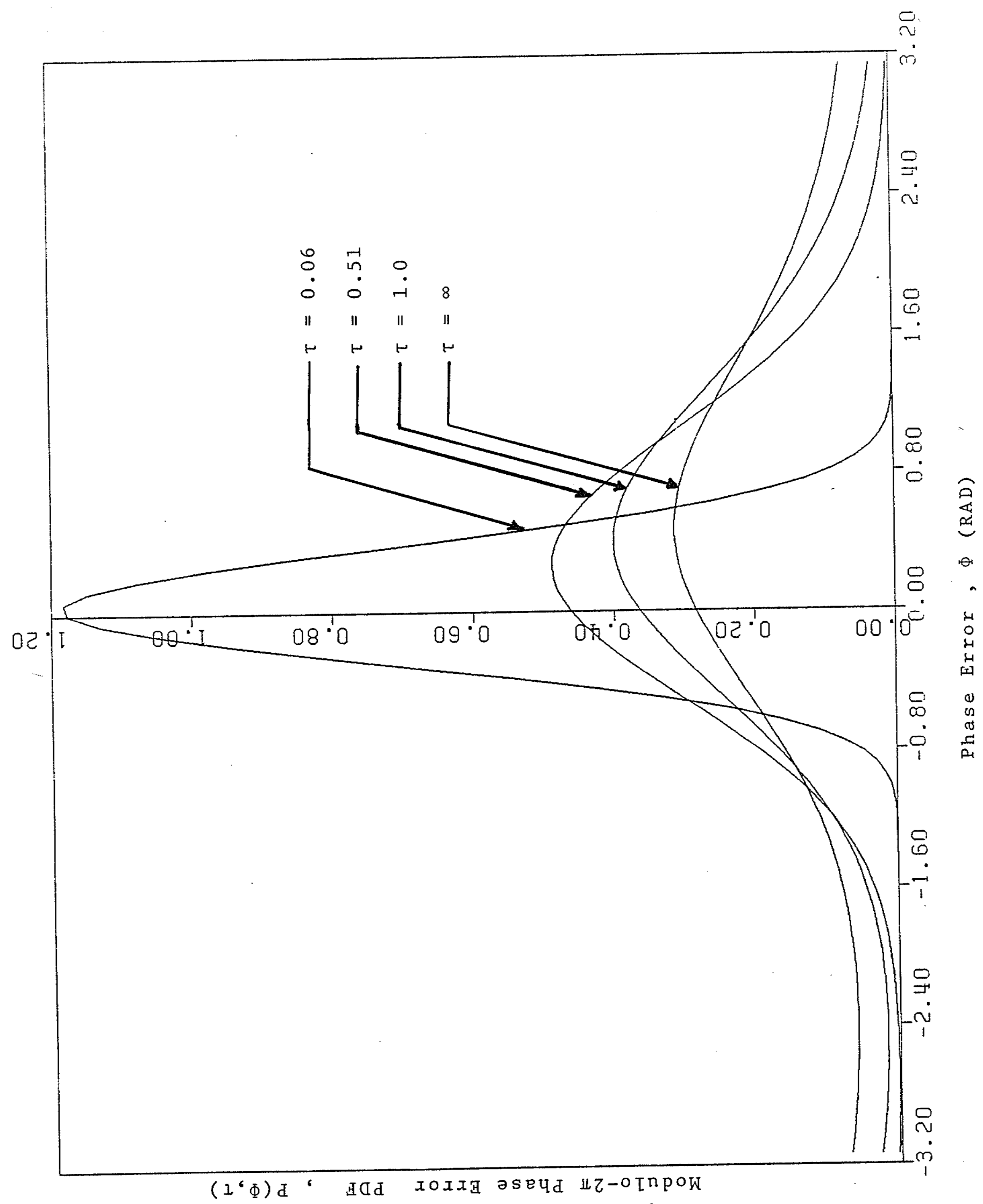

Figure 16. Modulo-2 $\pi$ Phase Error Probability Density Function

$$
\alpha=1.0, \Phi_{0}=0, \gamma=\sin \frac{\pi}{4} \text {. }
$$




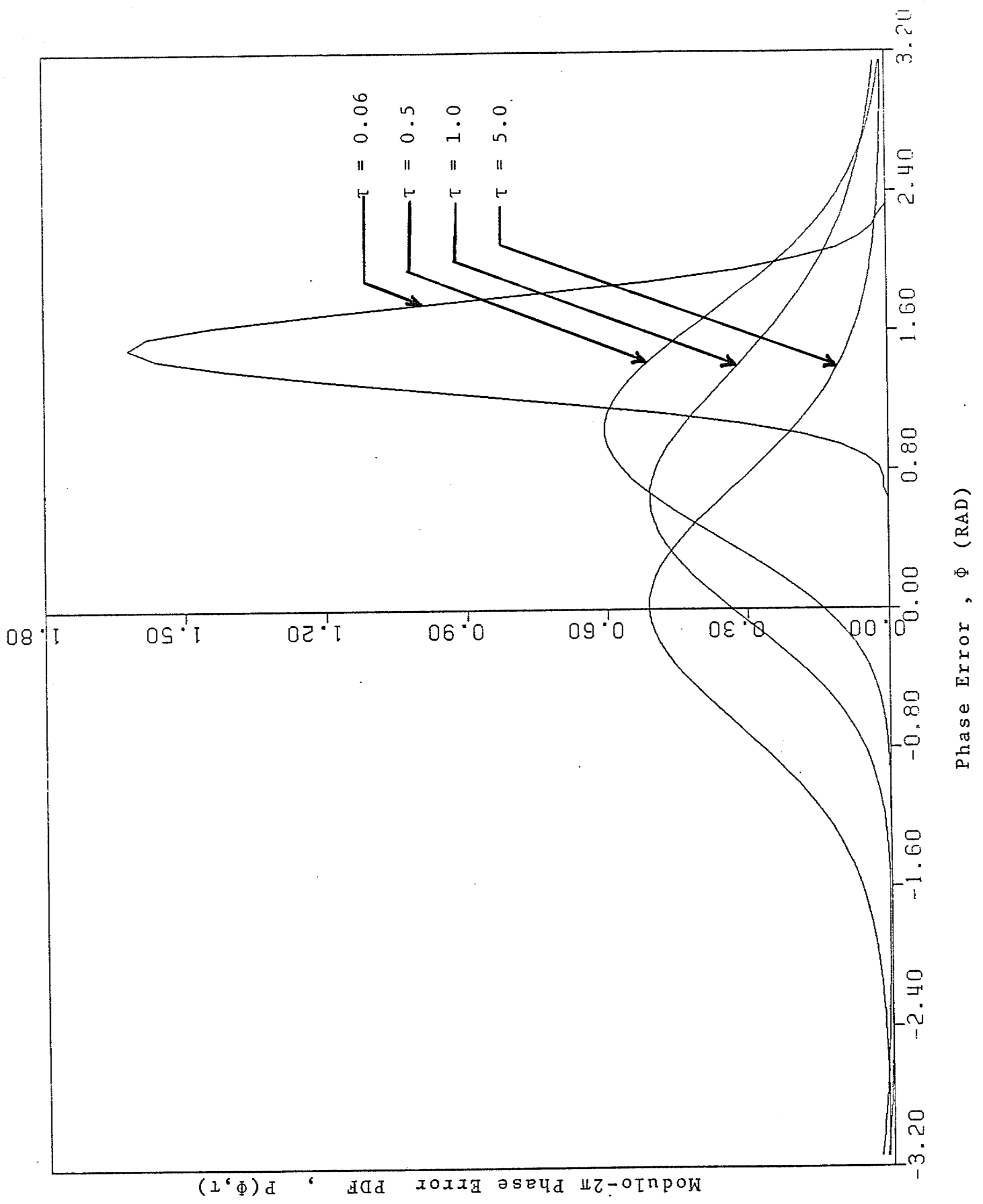

Figure 17. Modulo-2 $2 \pi$ Phase Error Probability Density Eunction $\alpha=2.0, \Phi_{0}=\frac{\pi}{2}, \quad \gamma=0$. 


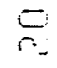

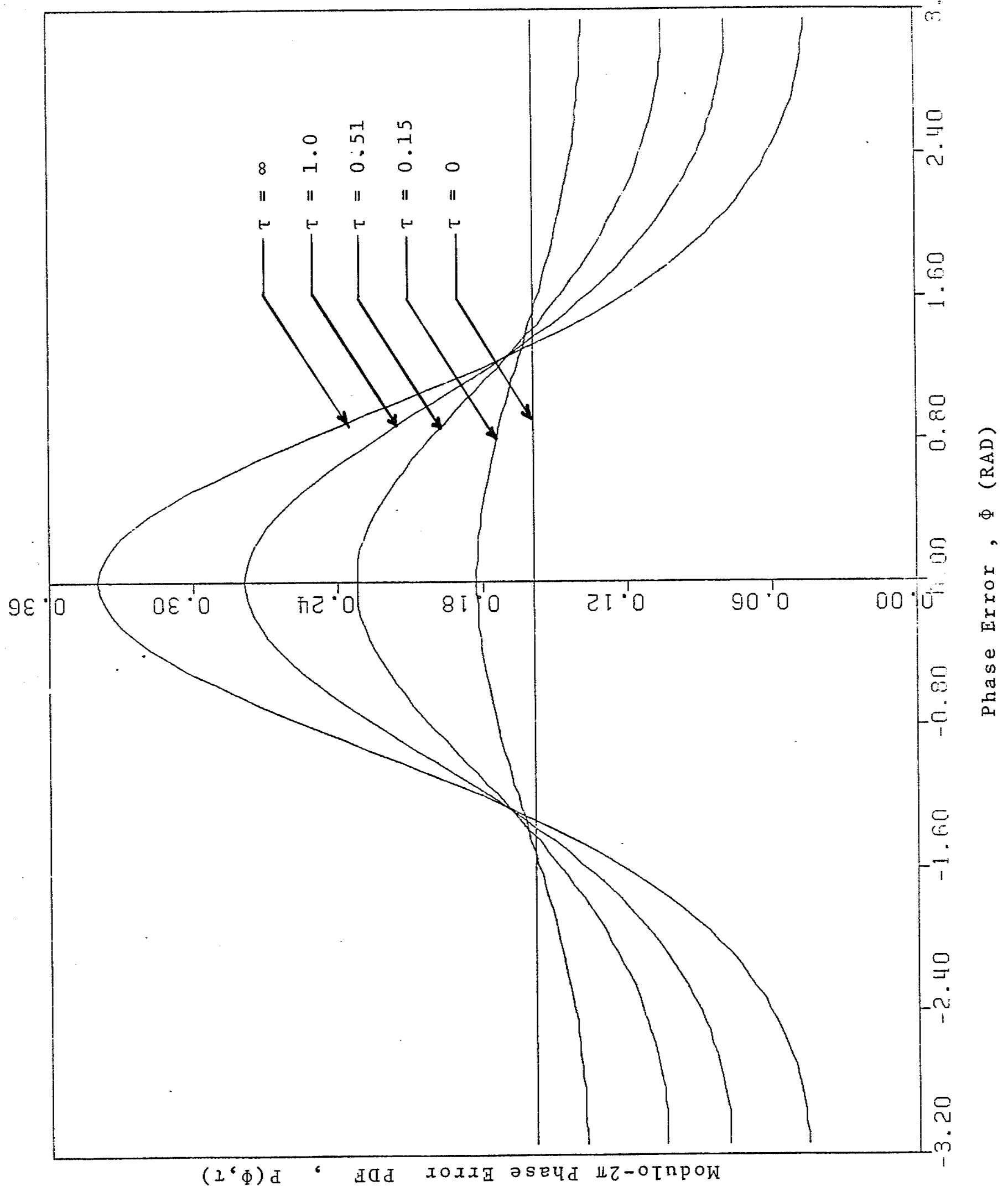

Figure 19. Modulo-2 $\pi$ Phase Error Probability Density Function $\alpha=1.0, \gamma=0, P(\Phi, 0)=\frac{1}{2 \pi}$. 


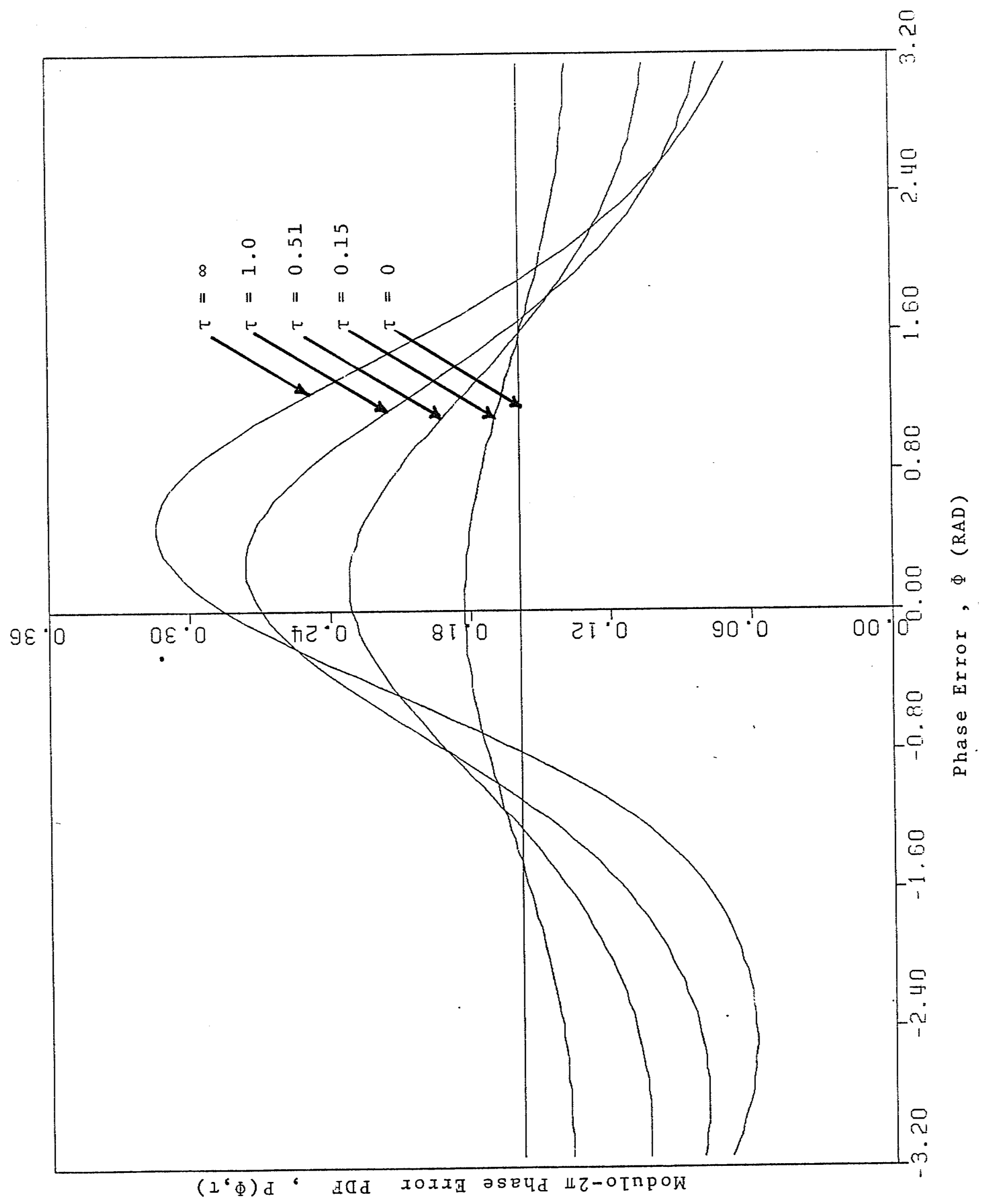

Figure 20. Modulo-2 $\pi$ Phase Error Probability Density Function $\alpha=1.0, \gamma=\sin \frac{\pi}{4}, P(\Phi, 0)=\frac{1}{2 \pi}$. 


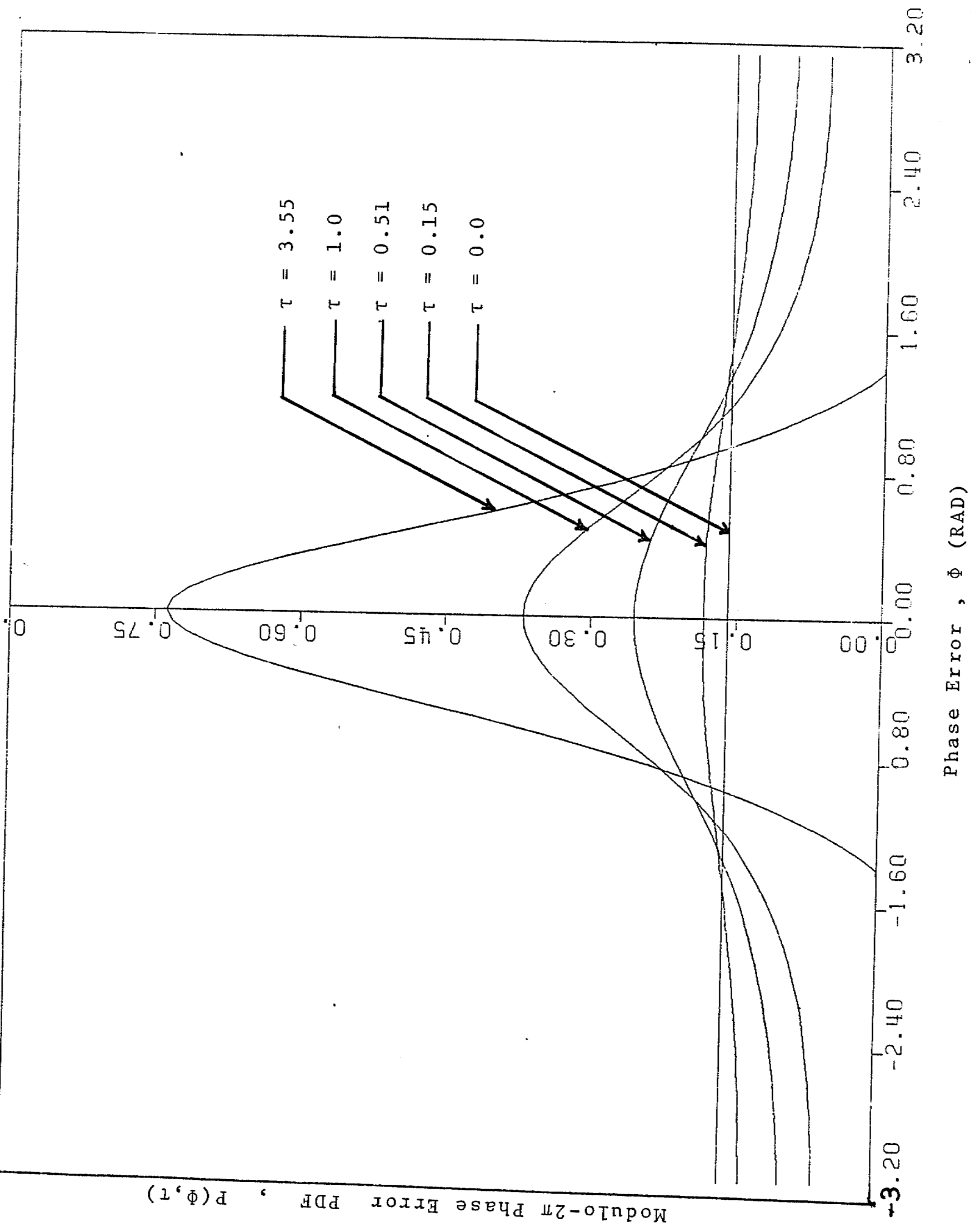

Figure 21. Modulo-2 2 Phase Error Probability Density Function $\alpha=5.0, \gamma=0 \quad, \quad P(\Phi, 0)=\frac{1}{2 \pi}$. 


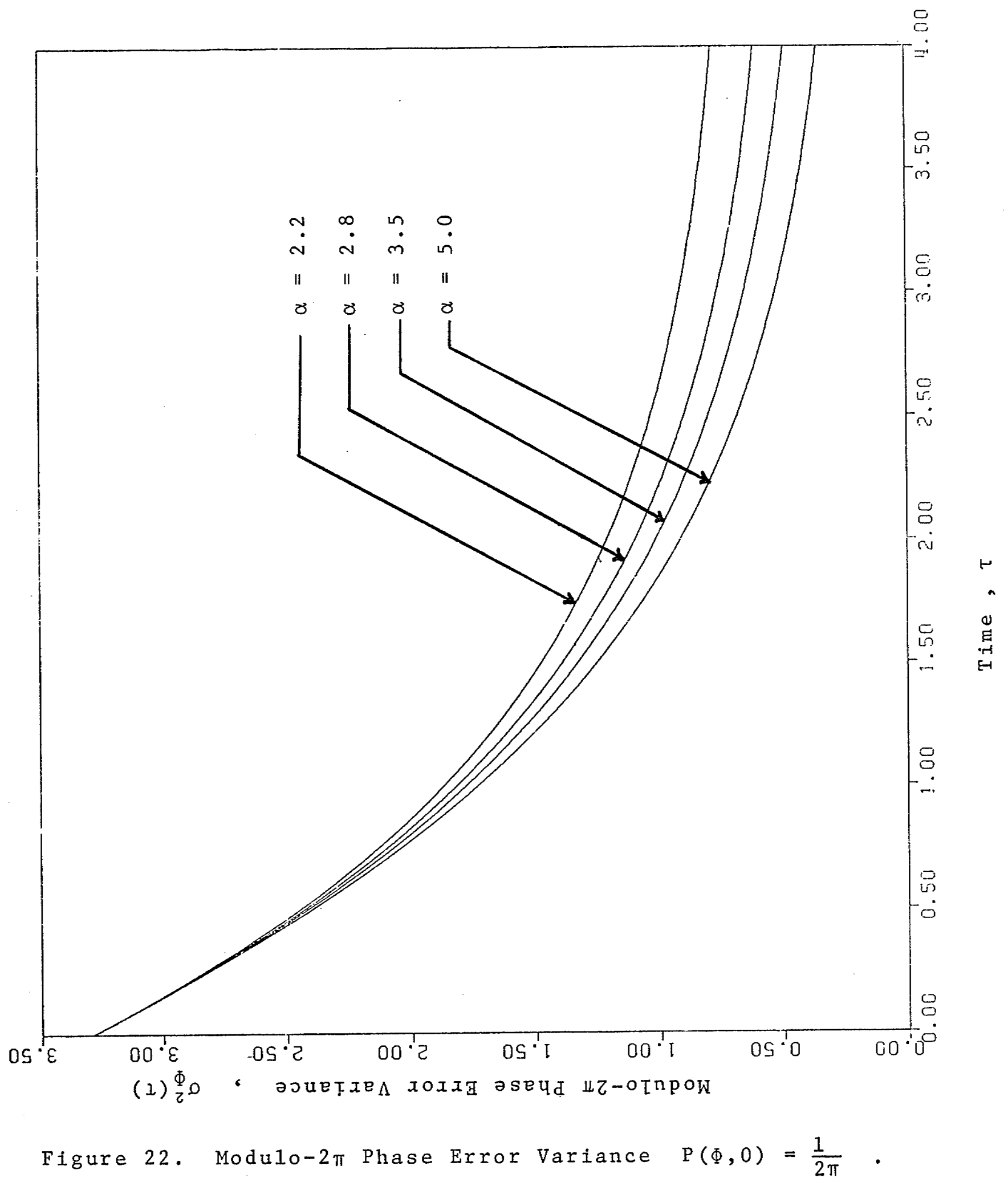




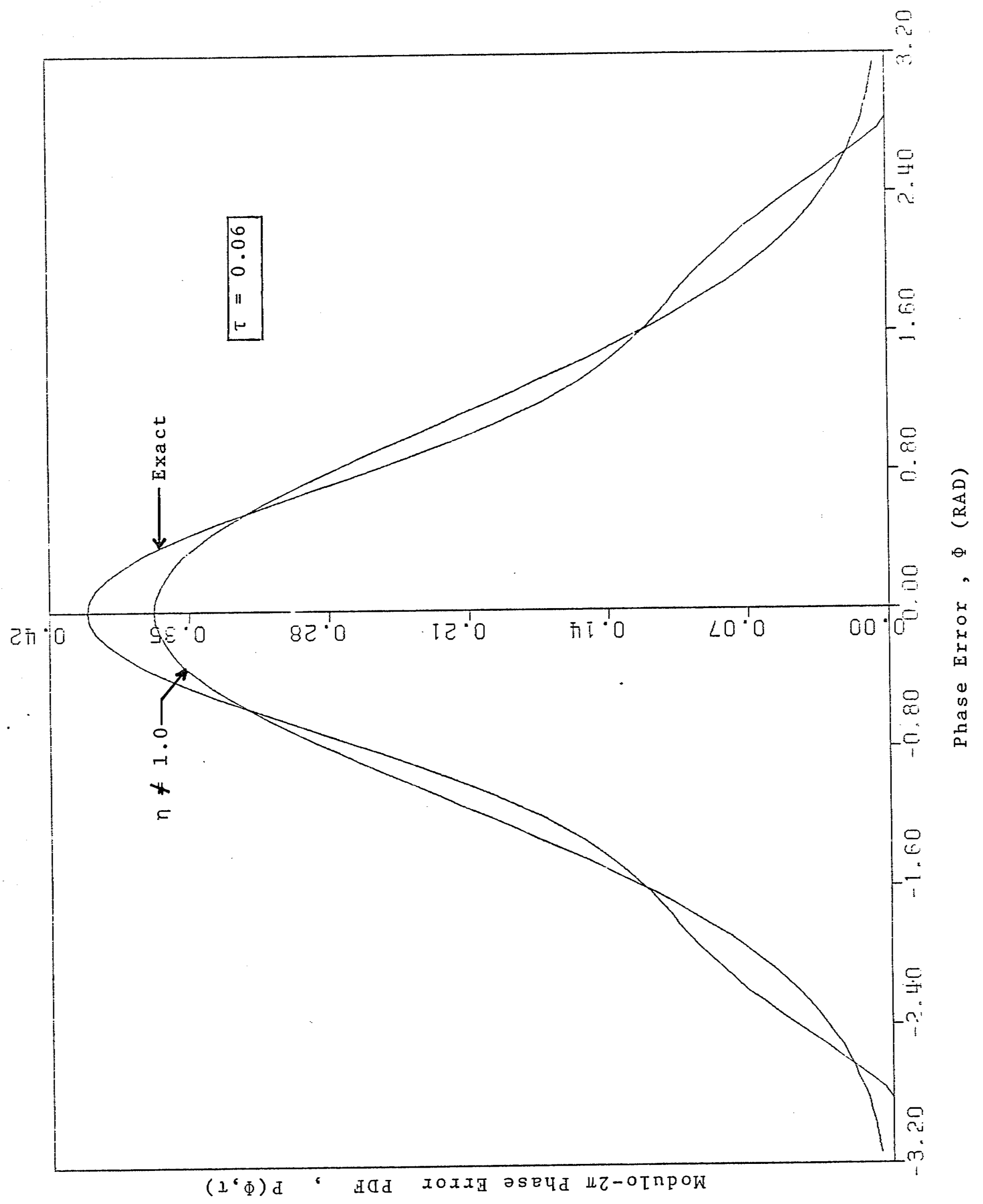

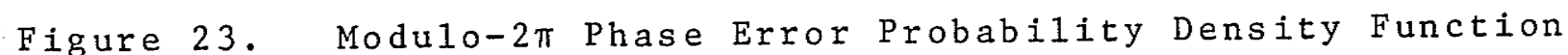
$\alpha=0.1, \Phi_{0}=0, \gamma=0$. 


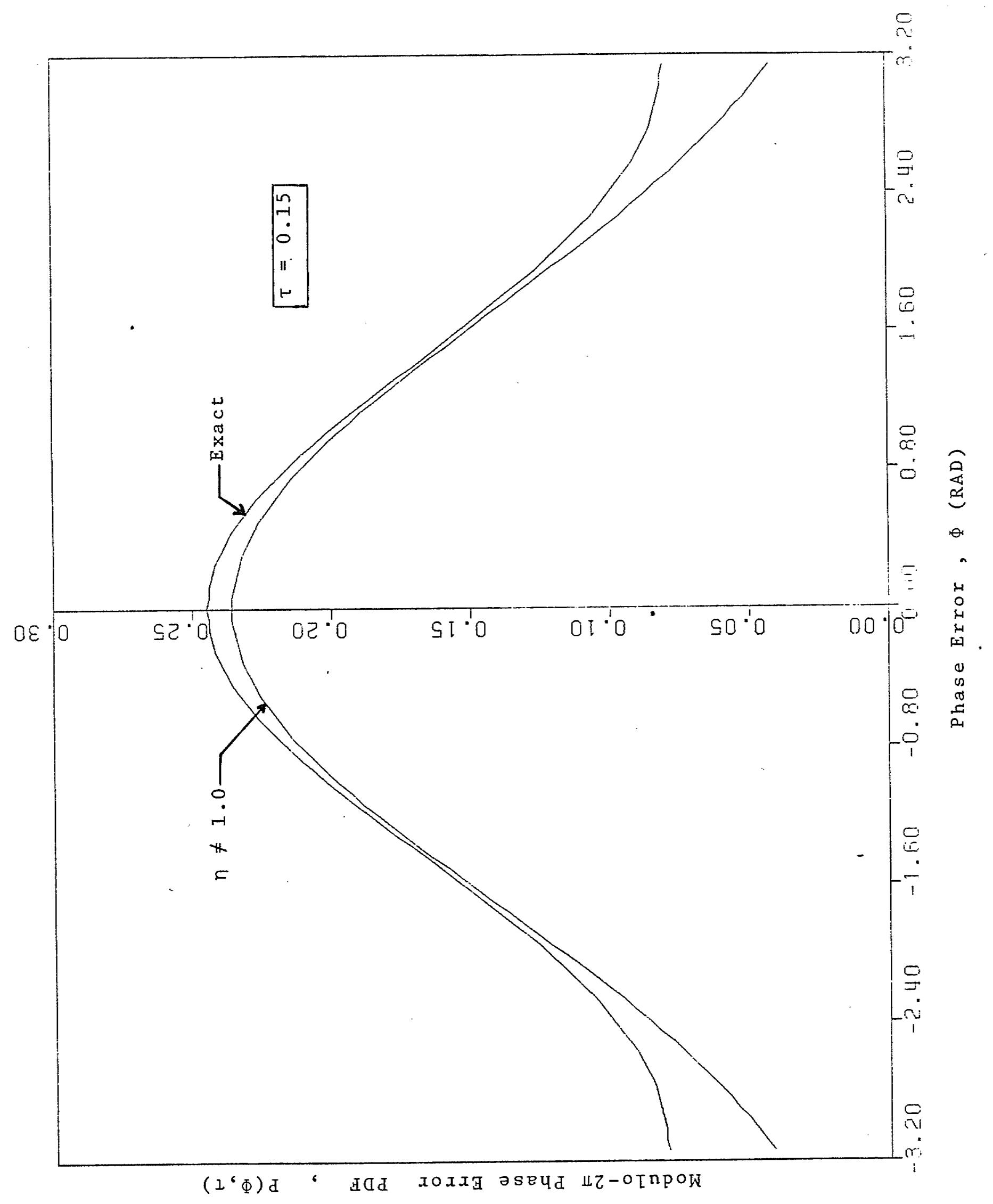

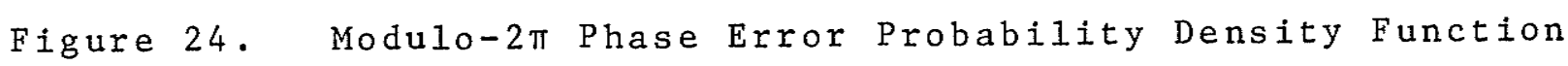
$\alpha=0.1, \Phi_{0}=0, \gamma=0$. 


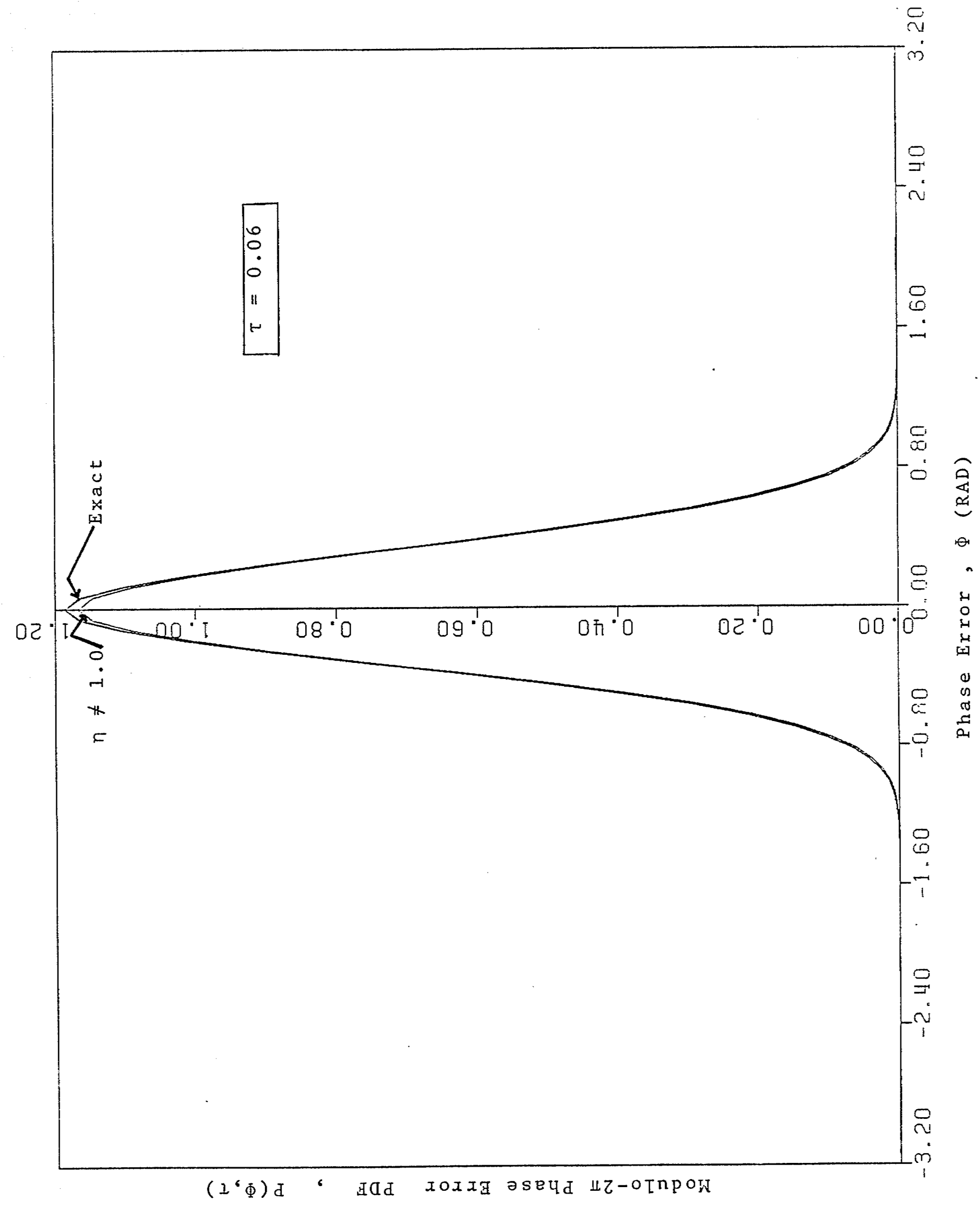

Figure 25. Modulo- $2 \pi$ Phase Error Probability Density Function $\alpha=1.0, \Phi_{0}=0, \gamma=0$. 


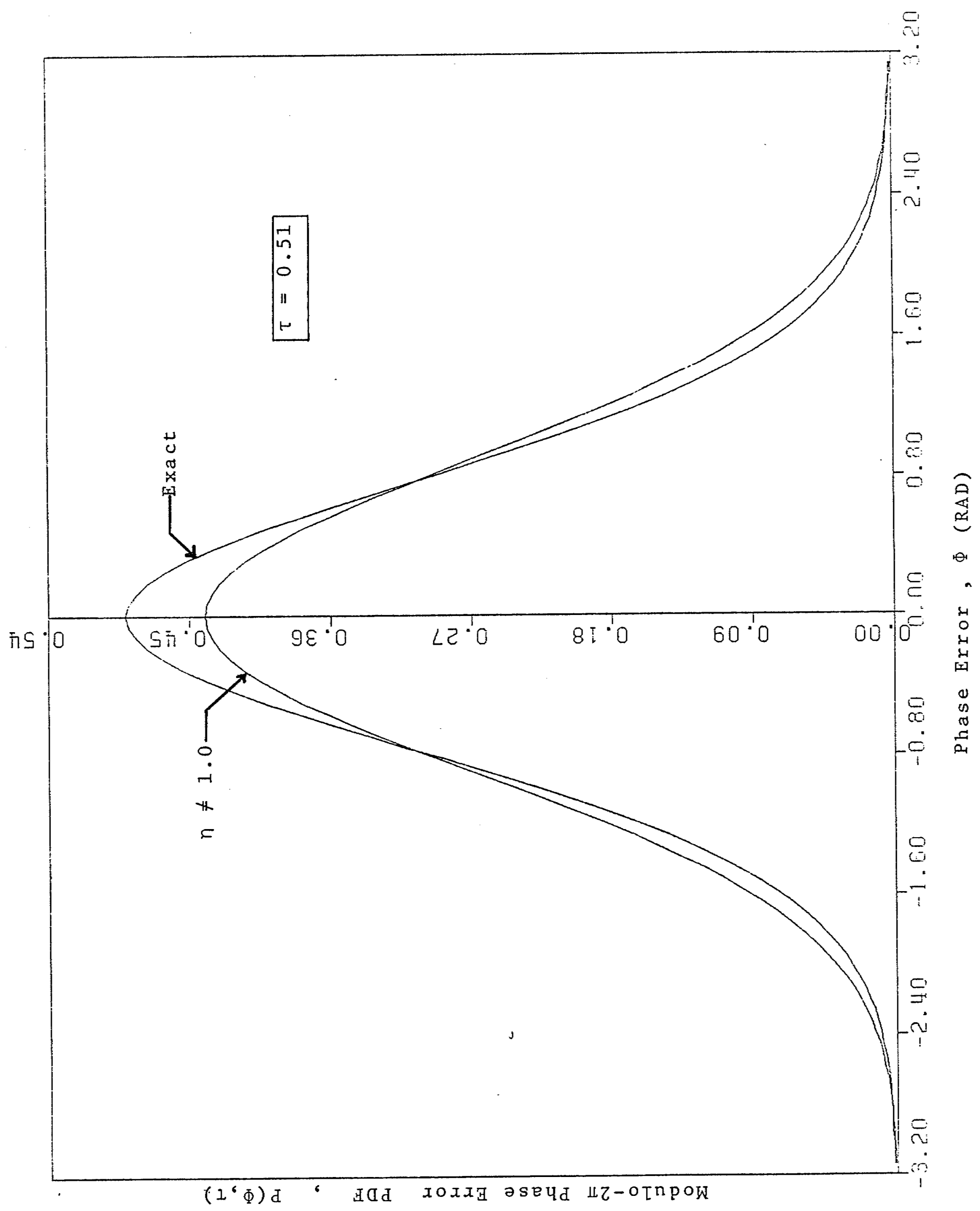

Eigure 26. Modulo- $2 \pi$ Phase Error Probability Density Function $\alpha=1.0, \Phi_{0}=0, \gamma=0$. 


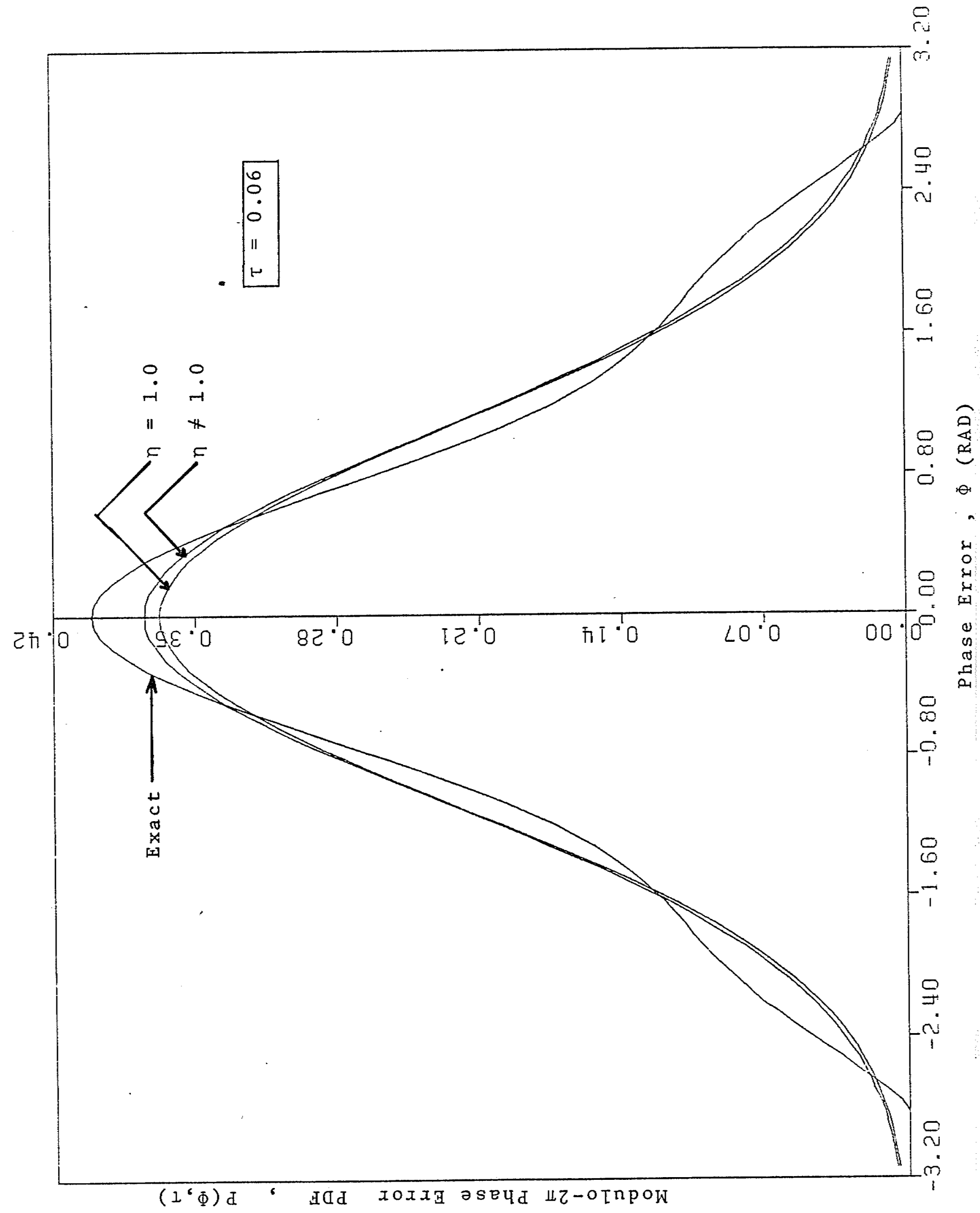

Figure 27. Modulo-2 2 Phase Error Probability Density Function $\alpha=0.1, \Phi_{0}=0, \gamma=0$. 


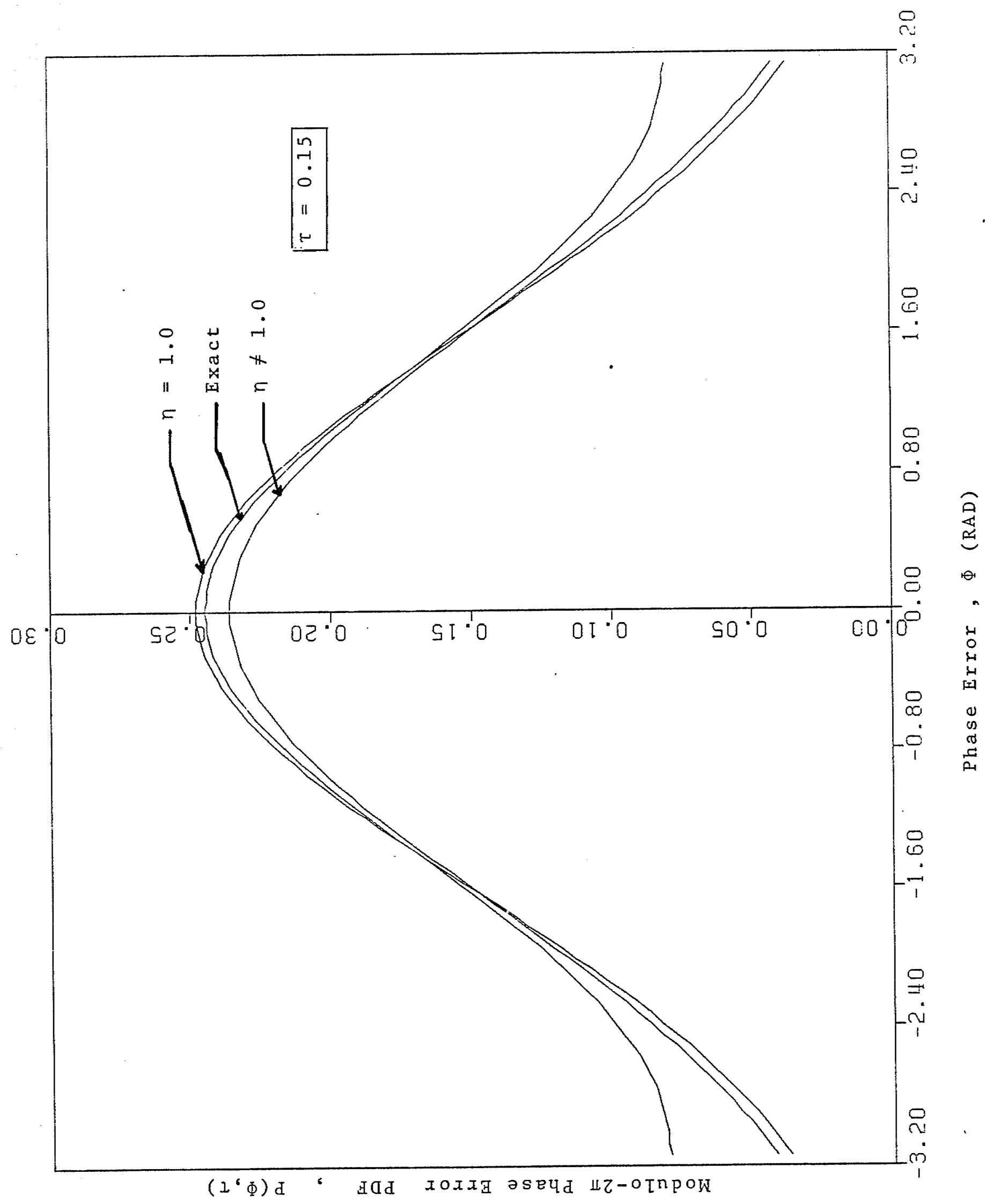

Figure 28. Modulo- $2 \pi$ Phase Error Probability Density Function $\dot{\alpha}=0.1, \Phi_{0}=0, \gamma=0$. 


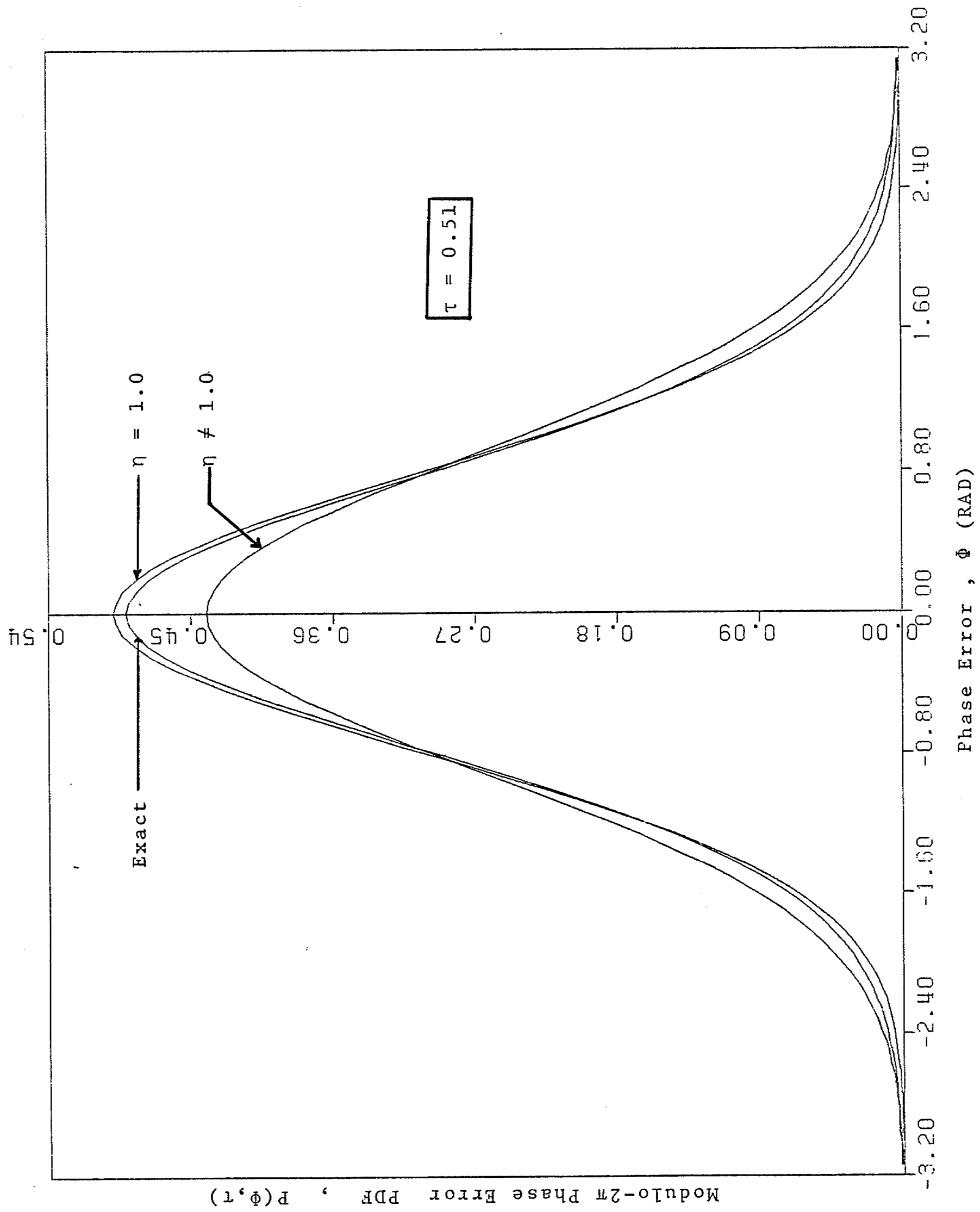

Figure 29. Modulo-2 2 Phase Error Probability Density Function $\alpha=1.0, \Phi_{0}=0, \quad \gamma=0$. 
CHAPTER VI

\section{CONCLUSION}

This study has resulted in a method of finding transient and steady state solutions for the Fokker-Planck equation as applied to the first-order phase-locked loop. The method provides analytic expressions for the modulo- $2 \pi$ phase error probability density function for special cases. For the general case, the thesis develops a technique (namely, the RLC ladder approach) which is used to find the transient and steady state modulo-2 $2 \pi$ phase error PDF numerically and analytically, respectively. Verification of this technique is demonstrated by comparison of the results obtained with those previously published. Unlike existing methods, the present technique provides a computationally efficient method for the determination of the transient statistics of the firstorder phase-1ocked 1oop. Although a specific numerical technique (CSMP) was used to solve the truncated RLC ladder. network, it is an advantage of this formulation that any of the well known network analysis methods could be used to obtain an analytical solution for the corresponding modulo- $2 \pi$ phase error PDF.

It is hoped that the RLC ladder approach presented in this thesis, may open up a new vista for future development of the analysis of the nonlinear phase-locked loop through the analysis of linear RLC ladder networks. In add- 
ition, it may also provide more insight to the transient

behaviour of the phase-1ocked loop.

The fact that the solutions for the special cases can be interpreted as solutions for infinite RLC ladder networks with special element values should also be of general interest.

The thesis also presents linearization methods which have resulted in a systematic procedure of obtaining the phase error transitional densities of first and second-order phase-1ocked 1oops without recourse to the Fokker-Planck technique. 


\section{REFERENCES}

[1] Gruen, W.J., "Theory of AFC Synchronization," Proc. IRE., IRE 41, pp. 1043-1048, 1953.

[2] Richman, D., "Color Carrier Reference Phase Synchronization Accuracy in NTSC Color TV," Proc. IRE., Ire 42, pp. $106-133,1954$.

[3] Viterbi, A.J., Principles of Coherent Communication, pp. 14-40,76-95 and 104-107, McGraw-Hil1, 1966.

[4] Gardner, F.M., Phaselock Techniques, pp. 1-70, John Wiley, 1966 .

[5] Panter, P.F., Modulation, Noise and Spectral Analysis, pp. 495-503, McGraw-Hi11, 1965.

[6] Schwartz, M., Bennett, W.R., and Stein, S., Communication systems and Techniques, pp. 161-163, McGraw-Hi11, 1966 .

[7] Van Trees, H.L., Detection, Estimation, and Modulation Theory, Part I, John Wiley, 1968 .

[8] Ibid., Detection, Estimation, and Modulation Theory, Part II, pp.:37-71, John Wiley, 1971 .

[9] Sakrison, D.J., Notes on Analog Communication, Ch. 5, Sec. 2, Van Nostrand, 1970 .

[10] Lindsey, W.C., Synchronization Systems in Communication and Control, pp. 65-117 and 667-673, Prentice-Hal1, 1972 .

[11] Tikhonov, V.I., "The effects of Noise on Phase-1ock Oscillator Operation," Automat i Telemakh., V.22, No.9, 1959. 
[12] Ibid., "Phase-lock Automatic Frequency Control Application in the Presence of Noise," Automat $i$ Telemakh., V.23, No.3, 1960 .

[13] Dominiak, K.W. and Pickholtz, R.L., "Transient Behavior of a Phase-locked Loop in the Presence of Noise," IEEE Trans. Communication Technology, Vol. Com-18, pp. 452456, August 1970 .

[14] Ohlson, J.E. and Rutherford, A., "Transient Statistics of the First-order Phase-1ocked Loop," IEEE Trans. Communication, Vol. , Com- , pp. 698-703, May 1974.

[15] Grandoni, F. and Mengali, U., Transient Phenomena in a Phase-locked Loop with a Noisy Reference, Paper presented at National Telemetry Conference on Digital Phase-1ocked Loops, Houston, Texas, pp. 32E-1-32E-5, December 4-6, 1972 .

[16] von Neumann, J. and Richtmyer, R.D., "On the Numerical Solution of Partial Differential Equations of Parabolic Type," Los Alamos Report LA657, 25 December 1947. Reprinted in John von Neumann, Collected Works, V.5, A.H. Taub, Ed., pp. 652-663, Macmi11an, 1963.

[17] Viterbi, A.J., "Phase-locked Loop Dynamics in the Presence of Noise by Fokker-Planck Techniques," Proc. IEEE, Vo1. 51, pp. 1737-1753, December 1963.

[18] Papoulis, A., Probability, Random Variables, and Stochastic Processes, Ch. 15, McGraw-Hi11, 1965.

[19] Ming Chen Wang and Uhlenbeck, G.E., "On the Theory of the Brownian Motion II," Reviews of modern physics, 
Vo1. 17, No. 2 and 3, pp. 323-342, April-July 1945.

[20] Fuller, A.T., "Analysis of Nonlinear Stochastic Systems by Means of the Fokker-Planck Equation," INT. J. Control, Vo1. 9, No. 6, pp. 603-655, June 1969.

[21] Dorovkin, P.P., Linear Operators and Approximation Theory, pp. 175-179, Hindustan Publishing Corp. (India), 1960.

[22] Ogata, K., Modern Control Engineering, pp. 716-734, Prentice-Ha11, 1970 .

[23] Oberhettinger, F., Fourier Expansions A Collection of Formulas, pp. 20, Academic Press, 1973 .

[24] Miller, K.S., Engineering Mathematics, Dover Publication, 1956

[25] Gradsteyn, I.S. and Ryzhik, I.M., Table of Integrals Series and Products, Academic Press, 1965.

[26] Cooke, R.G., Infinite Matrices and Sequence Spaces, Dover Publication, 1955.

[27] Mangulis, $V .$, Handbook of Series for Scientists and Engineers, Academic Press, 1965.

[28] Abramowitz, M. and Stegun; I.A., Handbook of Mathematical Functions, National Bureau of Standards, Applied Mathematics Series 55, June 1965.

[29] Aström, K.J., Introduction to Stochastic Control Theory, pp. 63-68, Academic Press, 1970 .

[30] Caughey, T.K. and Dienes, J.K., "Analysis of a Nonlinear First-order System with a White Noise Input," Journal of App1ied Physics, VoI. 32, No. 11, pp. 2476-2479, November 1961 . 
[31] Willsky, A.S., "Fourier Series and Estimation on the Circle with Applications to Synchronous Communcation Part II: Implementation," IEEE Trans. Information Theory, Vo1. IT-20, No. 5, pp. 584-590, September 1974.

[32] Peirce, B.0., A Short Table of Integrals, Ginn and Company, 1929.

[33] Sussman, G.J. and Stallman, R.M., "Heuristic Techniques in Computer - Aided Circuit Analysis," IEEE Trans. Circuit Theory, Vo1. CAS-22, No. 11, pp. 857-865, November 1975.

[34] Tanaka, M. and Hishinuma, C. and Mori, S., "Symbolic Formulation of Coefficients of the Characteristic Polynomial of a Restricted Class of RCL Networks," IEEE Trans. Circuit Theory, Vo1. CAS-22, No. 8, pp. 654-661, August 1975 .

[35] Kuh, E.S. and Rohrer, R.A., "The State Variable Approach to Network Analysis," Proc. IEEE, Vo1. 53, pp. 672-686, July 1965 .

[36] Boakye, K.A. and Wing, 0., "On the Analysis and Realization of Cascaded Transmission-line Networks in the Time Domain," IEEE Trans. Circuit Theory, Vol. CT-20, No. 3, pp. 301-307, May 1973 .

[37] Kaufman, K., Mann, H., and Vlach, J., "A Fast Procedure for the Analysis of Time-invariant Networks," IEEE Trans. Circuit Theory, Vol. CT-18, No. 6, pp. 739-741, November 1971 
[38] Dertouzos, M.L., Athans, M., Spann, R.N., and Mason, S.J., Systems, Networks, and Computation: Basic Concepts, McGraw-Hi11, 1972 .

[39] Anderson, B.D.O. and Vongpanitlerd, S., Network Analysis and Synthesis A Modern Systems Theory Approach, Prentice-Ha11, 1973 .

[40] Bashkor, T.R., "A Note on Ladder Network Analysis," IRE Trans. Circuit Theory, pp. 168-169, June 1961 .

[41] Oh1son, J., Private Communication.

[42] Director,S.W. , Roherer,R.A., Introduction to System Theory, Mcgraw-Hi11, 1966.

[43] Forsythe,G. , Moler,C.B. , Computer Solution of Linear Algebriac Systems, pp.7,Prentice-Hall, 1967.

[44] Martens,G.o., "Topological Formulas for State-Variable Analysis of RLC Networks," Proc. Sixth Annual Allerton Conference on Circuit and System Theory,pp.242-246, October 1968 . 
APPENDIX A

DETERMINATION OF THE MODAL MATRIX

Q IN EQUATION $(3.40)$

Let $U_{n}$ be the eigenvector corresponding to the eigenvalue $\lambda_{\mathrm{n}}$ of $\mathrm{B}$,

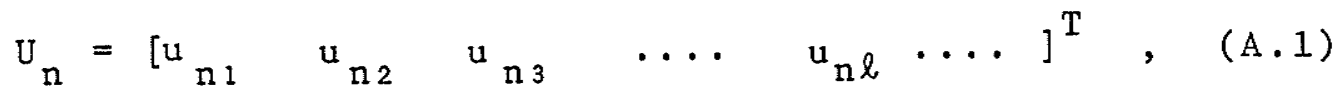

then $U_{n}$ must satisfy the equation

$\mathrm{BU}_{\mathrm{n}}=\lambda_{\mathrm{n}} \mathrm{U}_{\mathrm{n}}$

Introducing ( 3.36$)$ and (3.38) Into (A.2) yields,

$u_{n, \ell+1}=\frac{2(n-\ell)}{\ell(\ell+1)} u_{n, l}$

By letting the first entry of each eigenvector be unity;

that is, $u_{n_{1}}=1$ for $n=1,2,3, \ldots$, then from (A. 3)

$\mathrm{U}_{1}=\left[\begin{array}{llllllll}1 & 0 & 0 & 0 & \ldots & 0 & \ldots\end{array}\right]^{\mathrm{T}}, \mathrm{u}_{1 \ell}=0, \ell>1$,

$\mathrm{U}_{2}=\left[\begin{array}{llllllll}1 & 1 & 0 & 0 & \ldots & 0 & \ldots\end{array}\right]^{\mathrm{T}}, \mathrm{u}_{2 \ell}=0, \ell>2$,

$\mathrm{U}_{3}=\left[\begin{array}{llllllll}1 & 2 & \frac{2}{3} & 0 & \ldots & 0 & \ldots & ]^{\mathrm{T}}, \mathrm{u}_{3} l=0, \ell>3,\end{array}\right.$

$\mathrm{U}_{4}=\left[\begin{array}{lllllllll}1 & 3 & 2 & \frac{1}{3} & 0 & \ldots & 0 & \ldots\end{array}\right]^{\mathrm{T}}, \mathrm{u}_{4} l=0, \ell>4 . \quad(\mathrm{A} .4)$

By induction we obtain

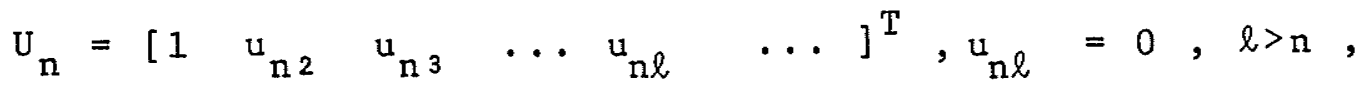

and

$$
u_{n \ell}=\frac{2^{\ell-1}(n-1) !}{\ell !(\ell-1) !(n-l) !}
$$


By definition

$$
Q=\left[\begin{array}{lllllll}
U_{1} & U_{2} & U_{3} & \cdots & U_{j} & \cdots
\end{array}\right] \quad \text { (A.6) }
$$

Hence,

$$
Q=\left\{q_{i j}\right\}=\left\{u_{j i}\right\}
$$

and

$$
q_{i j}=\frac{2^{i-1}(j-1) !}{i !(i-1) !(j-i) !}
$$


APPENDIX B

EVALUATing ThE CONSTANT $\left(\mathrm{d}_{\mathrm{n}}\right)$ OF EQUATION (3.42)

From (3.35) and (3.41) one obtains, for $\tau=0$,

$\sum_{n=r}^{\infty} \frac{(n-1) !}{(n-r) !} d_{n}=\frac{1}{\pi} \frac{(-1)^{r}(r-1) !}{2^{r-1}}$

By letting $n-r=m,(B .1)$ becomes

$\sum_{m=0}^{\infty} \frac{\Gamma(m+r)}{m !} d_{m+r}=\frac{2}{\pi}(-1)^{r} \frac{\Gamma(r)}{2^{r}}$

But from (3.43), for $z=1$,

$\sum_{m=0}^{\infty} \frac{(-1)^{m} \Gamma(m+r)}{m !}=\frac{\Gamma(r)}{2^{r}}$

Introducing (B. 3 ) into (B. 2 ) and comparing the result with (B.1) yie1ds,

$d_{m+r}=\frac{\pi}{2} \quad(-1)^{m+r}$

or
$\mathrm{d}_{\mathrm{n}}=(-1)^{\mathrm{n}} \frac{\pi}{2}$
(B. 4) 
APPENDIX C

$$
\begin{aligned}
& \text { SOLUTION OF THE SYSTEM OF EQUATIONS } \\
& \dot{C}_{n}(\tau)=\frac{n}{2} C_{n-1}(\tau)-n B C_{n}(\tau)-\frac{n}{2} C_{n+1}(\tau)
\end{aligned}
$$

Attempt a solution in the form

$$
C_{n}(\tau)=C \xi^{n}(\tau)
$$

where $C$ is a constant to be determined from

$$
c=c_{0}=\frac{1}{\pi}
$$

Introducing (C.1) and (C.2) into (3.83) and dividing both sides of the resulting equation by $\mathrm{Cn} \xi^{n-1}$ yields,

$$
\dot{\xi}=\frac{1}{2}-\beta \xi-\frac{1}{2} \xi^{2}
$$

(i) Consider the initial condition $\mathrm{C}_{n}(0)=0$, hence

$$
\xi(0)=0
$$

Integrating (C.3) keeping in mind the initial condition (C.4) yields, from [32],

$$
\begin{aligned}
& \xi(\tau)=\sqrt{1+\beta^{2}} \tanh \left\{\frac{\tau}{2} \sqrt{1+\beta^{2}}+\operatorname{arctanh}\left(\frac{\beta}{\sqrt{1+\beta^{2}}}\right)\right\}-\beta \cdot \text { (C.5) } \\
& \text { Introducing (C.5) and }(C .2) \text { into (C.1) yields, } \\
& C_{n}(\tau)=\frac{1}{\pi}\left\{\sqrt{1+\beta^{2}} \tanh \left[\frac{\tau}{2} \sqrt{1+\beta^{2}}+\operatorname{arctanh}\left(\frac{\beta}{\sqrt{1+\beta^{2}}}\right)\right]-\beta\right\}^{n} . \\
& \text { (C.6) }
\end{aligned}
$$

(ii) Consider the initial condition $C_{n}(0)=\frac{1}{\pi}$, hence 
$\xi(0)=1$

(C. 7 )

Integrating (C.3) with the initial condition (C.7) and introducing the result into (C.I) yields,

$C_{n}(\tau)=\frac{1}{\pi}\left\{a \frac{b \cosh \tau^{*}+a \sinh \tau^{*}}{a \cosh \tau^{*}+b \sinh \tau^{*}}-\beta\right\}^{n}$

where
$a=\sqrt{\beta^{2}+1}$
,
$b=\beta+1$
,

and

$$
\tau^{*}=\tau \sqrt{\beta^{2}+1}
$$

For $B=0$, equations $(C: 6)$ and (C.8) becomes, respectively,
$C_{n}(\tau)=\frac{1}{\pi}\left[\tanh \frac{\tau}{2}\right]^{n}$
$\mathrm{C}_{\mathrm{n}}(\tau)=\frac{1}{\pi}$
(C.10) 
APPENDIX D

DERIVATION OF EQUATION (3.49)

From [27] one obtains

$\sum_{n=0}^{\infty} r^{n} \cos (n \Phi+\delta)=\frac{\cos \delta-r \cos (\delta-\Phi)}{1-2 r \cos \Phi+r^{2}} \quad|r|<1 \ldots$ (D. 1$)$

For $\delta=0, \quad r=\tanh \frac{\tau}{2}$ and $\tau<\infty \quad$ (D.1) becomes.

$\sum_{n=0}^{\infty}\left[\tanh \frac{\tau}{2}\right]^{n} \cos n \Phi=\frac{1-\tanh \frac{\tau}{2} \cdot \cos \Phi}{1-2 \tanh \frac{\tau}{2} \cdot \cos \Phi+\tanh ^{2} \frac{\tau}{2}} \cdot$ (D.2)

Hence,

$$
\begin{aligned}
\sum_{n \geq 1}\left[\tanh \frac{\tau}{2}\right]^{n} \cos n \Phi & =\sum_{n=0}^{\infty}\left[\tanh \frac{\tau}{2}\right]^{n} \cos n \Phi-1 \\
& =\frac{\tanh \frac{\tau}{2} \cdot \cos \Phi-\tanh ^{2} \frac{\tau}{2}}{1-2 \cos \Phi \cdot \tanh \frac{\tau}{2}+\tanh ^{2} \frac{\tau}{2}} \cdot \text { (D. 3) }
\end{aligned}
$$

Introducing (D.3) into (3.47) yields, after some manipulation, $P(\Phi, \tau)=\frac{1}{2 \pi} \frac{\operatorname{sech}^{2} \frac{\tau}{2}}{1-2 \cos \Phi \cdot \tanh \frac{\tau}{2}+\tanh ^{2} \frac{\tau}{2}}$

which can be written as

$$
\mathrm{P}(\Phi, \tau)=\frac{1}{2 \pi} \frac{1}{\cosh \tau-\sinh \tau \cdot \cos \Phi} \quad \text { (D.4) }
$$




\section{APPENDIX E}

SOLUTION OF THE NONLINEAR, FIRST-ORDER, SIMULTANEOUS DIFFERENTAL EQUATIONS (3.56) AND (3.57)

Here the solution of equations (3.56) and ( 3.57 );

that is,

$$
\dot{g}=\frac{1}{2}\left(1-g^{2}\right) \cos f
$$

and

$$
\dot{\mathrm{f}}=\gamma-\frac{1+\mathrm{g}^{2}}{2 \mathrm{~g}} \operatorname{sinf}
$$

where $\dot{\mathrm{f}}=\frac{\mathrm{d} f}{\mathrm{~d} \tau}$ and $\dot{\mathrm{g}}=\frac{\mathrm{dg}}{\mathrm{d} \tau}$ are given.

From (E.I) and (E.2) one obtains

$$
\frac{\mathrm{dg}}{\mathrm{d} f}=\frac{\dot{g}}{\dot{\mathrm{f}}}=\frac{\frac{1}{2}\left(1-\mathrm{g}^{2}\right) \cos f}{\gamma-\frac{1+g^{2}}{2 g} \sin f}
$$

(E.3) can be written in the form

$$
M(f, g) d f+N(f, g) d g=0
$$

where

$$
M(f, g)=\frac{1}{2}\left(g^{2}-1\right) \cos f
$$

and

$$
N(f, g)=\gamma-\frac{1+g^{2}}{2 g} \operatorname{sinf} \quad \text { (E.5) }
$$

It is noted that (E.4) is not an exact differential equation since $\frac{\partial M}{\partial g} \neq \frac{\partial N}{\partial f}$. However, it can be rewritten as an exact differential equation as

$$
\mu \operatorname{Mdf}+\mu N d g=0
$$


where $\mu$ is an appropriate integration factor so that

$$
\frac{\partial}{\partial g}(\mu M)=\frac{\partial}{\partial f}(\mu N)
$$

It is found that $\mu$ which satisfies (E.7) is

$$
\mu=\frac{g}{\left(g^{2}-1\right)^{2}}
$$

Hence, a solution of the exact equation (E.6) can be found in the form

$$
\psi(f, g)=\text { constant }
$$

where $\frac{\partial \psi}{\partial g}=\mu N \quad$ and $\quad \frac{\partial \psi}{\partial f}=\mu M$.

From (E.5) and (E.10) one obtains

$$
\frac{\partial \psi}{\partial f}=\frac{\mu}{2}\left(g^{2}-1\right) \cos f
$$

and hence,

$$
\begin{aligned}
\psi & =\frac{\mu}{2}\left(g^{2}-1\right) \sin f+\xi(g) \\
& =\frac{g}{2\left(g^{2}-1\right)} \sin f+\xi(g)
\end{aligned}
$$

Differentiating $\psi$ in (E.II) with respect to $g$ and using (E.5), (E.8) and (E.10) yie $1 \mathrm{ds}$,

$$
\frac{\mathrm{d} \xi(g)}{\mathrm{dg}}=\frac{\gamma g}{\left(g^{2}-1\right)^{2}}
$$

Integrating (E.12) and introducing the result into (E.11) yields, 


$$
\begin{aligned}
\psi=\frac{g}{2\left(g^{2}-1\right)} \operatorname{sinf}-\frac{y}{2\left(g^{2}-1\right)} & =\text { constant } \\
& =\psi(f(0), g(0)) .
\end{aligned}
$$

From (3.53) and (E.13) one obtains

$$
\frac{g}{2\left(g^{2}-1\right)} \operatorname{sinf}-\frac{y}{2\left(g^{2}-I\right)}=\frac{\gamma}{2}
$$

hence,

$$
g=\frac{1}{\gamma} \sin f
$$

Introducing (E.14) into (E.2) yields,

$$
\dot{\mathrm{f}}=\frac{1}{2 \gamma}\left(\gamma^{2}-\sin ^{2} \mathrm{f}\right)
$$

or

$$
\int_{0}^{f} \frac{d f}{\gamma^{2}-\sin ^{2} f}=\frac{1}{2 \gamma} \int_{0}^{\tau} d \tau
$$

Integrating both sides of (E.16) [25] yields,

$$
\begin{aligned}
& f(\tau)=\arctan \left[\frac{\gamma}{\sqrt{\gamma^{2}-1}} \tan \right. \\
& g(\tau)=\frac{\sin \left(\frac{\tau}{2} \sqrt{\gamma^{2}-1}\right)}{\sqrt{\gamma^{2}-\cos ^{2}\left(\frac{\tau}{2} \sqrt{\gamma^{2}-1}\right)}}
\end{aligned}
$$

and

for $\gamma^{2}>1$

$$
f(\tau)=\arctan \left(\frac{\tau}{2}\right)
$$

and

$$
g(\tau)=\frac{\tau}{\sqrt{4+\tau^{2}}}
$$

for $\gamma^{2}=1 \quad$, and

$$
f(\tau)=\arctan \left\{\frac{\gamma}{\sqrt{1-\gamma^{2}}} \tanh \left(\frac{\tau}{2} \sqrt{1-\gamma^{2}}\right)\right\},
$$


and

$$
g(\tau)=\frac{\sinh \left(\frac{\tau}{2} \sqrt{1-\gamma^{2}}\right)}{\sqrt{\cosh ^{2}\left(\frac{\tau}{2} \sqrt{1-\gamma^{2}}\right)-\gamma^{2}}}
$$

for $\gamma^{2}<1$. 


\section{APPENDIX F}

DERIVATIONS OF EQUATIONS

$$
(3.64),(3.65) \text { and }(3.66)
$$

The result of introducing (3.9) and (3.52) into (3.6)

is

$$
\begin{aligned}
P(\Phi, \tau) & =\frac{1}{2 \pi}+\frac{1}{\pi} \sum_{n \geq 1}[g(\tau)]^{n}\{\cos n f(\tau) \cos n \Phi+\operatorname{sinn} f(\tau) \operatorname{sinn} \Phi\} \\
& =\frac{1}{2 \pi}+\frac{1}{\pi} \sum_{n \geq 1}[g(\tau)]^{n} \cos n(\Phi-f(\tau))
\end{aligned}
$$

From [27] one obtains,

$$
\sum_{n \geq 1} r^{n} \cos (n X+\delta)=\frac{r \cos (\delta+X)-r^{2} \cos \delta}{1-2 r \cos x+r^{2}},|r|<1 \quad \text { (F.2) }
$$

Letting $\delta=0, \quad X=\Phi-f(\tau)$ and $r=g(\tau) \quad(F .2)$ becomes, for $\tau<\infty$,

$\sum_{n \geq 1}[g(\tau)]^{n} \cos n(\Phi-f(\tau))=\frac{g(\tau) \cos (\Phi-f(\tau))-g^{2}(\tau)}{1-2 g(\tau) \cos (\Phi-f(\tau))+g^{2}(\tau)} \quad(F \cdot 3)$ Introducing (F.3) into (F.1) yields,

$$
P(\Phi, \tau)=\frac{1}{2 \pi} \frac{1-g^{2}(\tau)}{I-2 g(\tau) \cos (\Phi-f(\tau))+g^{2}(\tau)}
$$

For $\gamma<1$

Introducing (3.58) and (3.59) into (F.4) and making use of the trigenometric identity $\cos (\Phi-f)=\cos f[\cos \Phi+\tan f \cdot \sin \Phi]$ yields, 


$$
\begin{aligned}
& P(\Phi, \tau)=\frac{1}{2 \pi} \text {. } \\
& \frac{1-\gamma^{2}}{\cosh ^{2} \frac{\tau_{1}}{2}-\gamma^{2}-2 \sqrt{1-\gamma^{2}} \sinh \frac{\tau_{1}}{2} \cosh \frac{\tau_{1}}{2}\left[\cos \Phi+\left(\frac{\gamma}{\sqrt{1-\gamma^{2}}} \tanh \frac{\tau_{1}}{2}\right) \sin \Phi+\sinh ^{2} \frac{\tau_{1}}{2}\right.} \\
& =\frac{1}{2 \pi} \frac{1-\gamma^{2}}{\cosh \tau_{1}-\gamma^{2}-\sqrt{1-\gamma^{2}} \sinh \tau_{1} \cdot \cos \Phi+\gamma\left(1-\cosh \tau_{1}\right) \sin \Phi} . \\
& \text { where } \tau_{1}=\tau \sqrt{1-\gamma^{2}} \quad, \quad \tau<\infty \\
& \text { (F.5) } \\
& \text { for } Y>1
\end{aligned}
$$

$$
\text { Introducing }(3.60) \text { and }(3.61) \text { into (F.4) and following }
$$
the same manipulations which produced (F.5) we obtain

$$
\mathrm{P}(\Phi, \tau)=\frac{1}{2 \pi}
$$$$
\frac{\gamma^{2}-1}{\gamma^{2}-\cos \tau_{2}-\sqrt{\gamma^{2}-1} \sin \tau_{2} \cos \Phi+\gamma\left(1-\cos \tau_{2}\right) \sin \Phi} \quad \text { (F.6) }
$$$$
\text { where } \tau_{2}=\tau \sqrt{\gamma^{2}-1} \quad, \quad \tau<\infty
$$$$
\text { for } \gamma=1
$$

$$
\text { Introducing (3.62) and (3.63) into (F.4) and making use }
$$
of $\cos (\Phi-f)=\cos f \cdot \cos \Phi+\operatorname{sinf} \cdot \sin \Phi$ yields,

$$
P(\Phi, \tau)=\frac{1}{\pi\left(\tau^{2}+2-2 \tau \cos \Phi-\tau^{2} \sin \Phi\right)} \quad, \quad \tau<\infty .(F .7)
$$


APPENDIX G

DERIVATIONS OF EQUATIONS $(4.25)-(4.29)$

From $(4.20),(4.22)$ and $(4.24)$ one obtains

$\frac{\mathrm{dm}}{\mathrm{dt}}=\mathrm{m}_{2}$

and

$$
\frac{\mathrm{dm}_{2}}{\mathrm{dt}}=-\mathrm{KAam}_{1}-\mathrm{KAm}_{2}
$$

with the initial conditions

$$
\mathrm{m}_{1}(0)=\mathrm{x}_{0}(0) \text { and } \mathrm{m}_{2}(0)=\mathrm{x}_{1}(0) \text { (G.3) }
$$

Differentiating (G.2) with respect to $t$ and using (G.1) yields,

$$
\frac{\mathrm{d}^{2} \mathrm{~m}_{2}}{\mathrm{dt}^{2}}+\mathrm{KA} \frac{\mathrm{dm}}{\mathrm{dt}}+\mathrm{KAam}_{2}=0
$$

(G.4) is a linear, second-order, ordinary differential equation. The solution of (G.4) which satisfies the initial condition (G.3) can easily be obtained

$$
m_{2}(t)=e^{-A K t / 2}\left[x_{1}(0)\left(\cos \Omega t-\frac{A K}{2} \frac{\sin \Omega t}{\Omega}\right)-x_{0}(0) A K a \frac{\sin \Omega t}{\Omega}\right]
$$

where $\Omega=\sqrt{\mathrm{KAa}-\frac{\mathrm{K}^{2} \mathrm{~A}^{2}}{4}}$.

Integrating (G.5) and using (G.3) yields,

$$
m_{1}(t)=e^{-A K t / 2}\left[\frac{\sin \Omega t}{\Omega} x_{1}(0)+\left(\cos \Omega t+\frac{A K}{2} \frac{\sin \Omega t}{\Omega}\right) x_{0}(0)\right] .
$$


From $(4.20),(4.23)$ and $(4.24)$ one obtains

$$
\begin{aligned}
& \dot{\mathrm{V}}_{1}=\frac{\mathrm{dV}_{1}}{\mathrm{dt}}=2 \mathrm{~V}_{2} \\
& \dot{\mathrm{V}}_{2}=\frac{\mathrm{dV}_{2}}{\mathrm{dt}}=-\mathrm{KAaV}_{1}-\mathrm{KAV}_{2}+\mathrm{V}_{3} \\
& \dot{\mathrm{V}}_{3}=\frac{\mathrm{dV}_{3}}{\mathrm{dt}}=-2 \mathrm{KAaV}_{2}-2 \mathrm{KAV}_{3}+\frac{\mathrm{N}_{0}}{2} \mathrm{~K}^{2}
\end{aligned}
$$

Differentiating (G.8) once w.r.t. $t$ and using (G.7) and (G.9) yields,

$$
V_{3}=\frac{\frac{\mathrm{N}_{0} \mathrm{~K}^{2}}{2}-4 \mathrm{KAaV}_{2}-\mathrm{KA}_{2}-\ddot{\mathrm{V}}_{2}}{2 \mathrm{KA}}
$$

Differentiating (G.8) twice w.r.t $t$ and using (G.7), (G.9) and (G.10) yie1ds,

$$
\frac{d^{3} V}{d t^{3}}+3 K A \frac{d^{2} V}{d t^{2}}+2 K A(K A+2 a) \frac{d V}{d t}+4 K^{2} A^{2} a V_{2}=0
$$

Solution of (G.11) with the initial conditions

$$
\mathrm{V}_{2}(0)=\dot{\mathrm{V}}_{2}(0)=0 \text { and } \quad \ddot{\mathrm{V}}_{2}(0)=\frac{\mathrm{N}_{0} \mathrm{~K}^{2}}{4}
$$

yie1ds,

$$
V_{2}(t)=\frac{N_{0} K^{2}}{4} e^{-A K t} \frac{\sin ^{2} \Omega t}{\Omega^{2}}
$$

Using (G.13) in (G.7) and considering the initial condition

$$
V_{1}(0)=0
$$

yields,

$$
\left.V_{1}(t)=\frac{N_{0}}{4 A^{2} a}\left[1-e^{-A K t}\left(1+\frac{A^{2} K^{2}}{2} \frac{\sin ^{2} \Omega t}{\Omega^{2}}+A K \frac{\sin 2 \Omega t}{2 \Omega}\right)\right] \cdot \text { (G. } 15\right)
$$


Introducing (G.13) and (G.15) into (G.9) then integrating and using $\mathrm{v}_{3}(0)=0$ yields,

$$
V_{3}(t)=\frac{N_{0} K}{4 A}\left\{1-e^{-A K t}\left[1+\frac{A^{2} K^{2}}{2} \frac{\sin ^{2} \Omega t}{\Omega^{2}}-\frac{A K}{2} \frac{\sin 2 \Omega t}{\Omega}\right]\right\} .
$$




\section{APPENDIX H}

\section{DERIVATION OF THE FOKKER-PLANCK EQUATION [3]}

Consider

$$
I=\int_{-\infty}^{\infty} R(\phi) \frac{\partial p\left(\phi \mid \phi_{0}, t\right)}{\partial t} d \phi
$$

where $R(\phi)$ is an arbitrary analytic function whose derivatives should satisfy certain conditions, to be stated below.

$$
I=\lim _{\Delta t \rightarrow 0} \int_{-\infty}^{\infty} R(\phi) d \phi \frac{p\left(\phi \mid \phi_{0}, t+\Delta t\right)-p\left(\phi \mid \phi_{0}, t\right)}{\Delta t} \cdot(H, 2)
$$

Introducing (2.9) into (H.2) yields,

$$
\begin{aligned}
I & =\lim _{\Delta t \rightarrow 0} \frac{1}{\Delta t}\left[\int_{-\infty}^{\infty} R(\phi) d \phi \int_{-\infty}^{\infty} p\left(z \mid \phi_{0}, t\right) p(\phi \mid z, \Delta t) d z\right. \\
& \left.-\int_{-\infty}^{\infty} R(z) p\left(z \mid \phi_{0}, t\right) d z\right]
\end{aligned}
$$

Interchanging the order of integration and expanding the analytic function $R(\phi)$ in a Taylor series about $z$ yields,

$$
I=\sum_{n \geq 1} \frac{1}{n !} \int_{-\infty}^{\infty} R^{(n)}(z) D_{n}(z) p\left(z \mid \phi_{0}, t\right) d z
$$

where

$$
R^{(n)}(z)=\frac{d^{n} R(z)}{d z^{n}},
$$

and

$$
D_{n}(z)=\lim _{\Delta t \rightarrow 0} \frac{1}{\Delta t} \int_{-\infty}^{\infty}(\phi-z)^{n} p(\phi \mid z, \Delta t) d \phi \quad n=1,2,3, \ldots
$$


Assume that $R(z)$ and its derivatives decrease sufficiently rapidiy as $z \rightarrow \pm \infty$, so that

$$
\begin{aligned}
& \left.R^{(n-1)}(z) D_{n}(z) p\left(z \mid \phi_{0}, t\right)\right|_{-\infty} ^{\infty}=0 \\
& \left.R^{(n-2)}(z) \frac{\partial}{\partial z}\left[D_{n}(z) p\left(z \mid \phi_{0}, t\right)\right]\right|_{-\infty} ^{\infty}=0 \\
& \left.R(z) \frac{\partial^{n-1}}{\partial z^{n-1}}\left[D_{n}(z) p\left[z \mid \phi_{0}, t\right)\right]\right|_{-\infty} ^{\infty}=0
\end{aligned}
$$

Integrating the $\mathrm{n}^{\text {th }}$ term of the sum (H.4) by parts $n$ times, and after subtracting (H.4) from (H.1) and replacing the variable of integration. in (H.4) with $\phi$, one obtains

$$
\int_{-\infty}^{\infty} R(\phi) d \phi\left\{\left[\frac{\partial p\left(\phi \mid \phi_{0}, t\right)}{\partial t}\right]-\sum_{n \geq 1} \frac{(-1)^{n}}{n !} \frac{\partial^{n}}{\partial \phi^{n}}\left[D_{n}(\phi) p\left(\phi \mid \phi_{0}, t\right)\right]\right\}=0 .
$$

Since $R(\phi)$ was an arbitrary function except for the above conditions on its derivatives, in order for the integral to vanish, the quantity in brackets must also vanish.

Hence,

$\frac{\partial p\left(\phi \mid \phi_{0}, t\right)}{\partial t}=\sum_{n \geq 1} \frac{(-1)^{n}}{n !} \frac{\partial^{n}}{\partial \phi^{n}}\left[D_{n}(\phi) p\left(\phi \mid \phi_{0}, t\right)\right]$

where

$$
\begin{aligned}
D_{n}(\phi) & =\lim _{\Delta t \rightarrow 0} \frac{1}{\Delta t} \int_{-\infty}^{\infty}(\Delta z)^{n} p(\Delta z \mid z) d \Delta z \\
& =\lim _{\Delta t \rightarrow 0} \frac{E\left[(\Delta z)^{n} \mid z\right]}{\Delta t}
\end{aligned}
$$


APPENDTX T.

JUSTIFICATION OF 'IRUNCATION OF THE INRTNTE

SYSTEM (3.13)

The approximation of the infinite system (3.1.3), for $\gamma=0$, by a finite system of order $N$ can be justified by using a bound on $a_{N+1}$ (for a suitably chosen $N$ ) to show that the erroxs due to truncation are negligible. We proceed as follows : Suppose $a_{\mathrm{N}+1}$ is known, then all the variables $a_{n}(\tau)(1 \leq n \leq N)$ can be determined by solving the finite system of equations :

$$
\begin{aligned}
& \dot{a}_{1}(\tau)=-\beta a_{1}-\frac{1}{2} a_{2}+\frac{1}{2 \pi} \\
& \dot{a}_{2}(T)=a_{1}-4 \beta a_{2}-a_{3} \\
& \ldots \ldots \quad \ldots \ldots \\
& \ldots \\
& \dot{a}_{N}(\tau)=\frac{N}{2} a_{N-1}-N^{2} B a_{N}-\frac{N}{2} a_{N+1}
\end{aligned}
$$

which are the state equation for the ladder network consisting of the first $N$ sections shown in Fig. 30 .

System (I.1) can be vritten in matrix form as

$$
\dot{a}=A a+B_{1} U_{1}+B_{2} U_{2}
$$

where

$$
\begin{aligned}
& \mathrm{a}=\left[\begin{array}{lllll}
\mathrm{a}_{1} & \mathrm{a}_{2} & \mathrm{a}_{3} & . & \mathrm{a}_{\mathrm{N}}
\end{array}\right]^{\mathrm{T}}, \\
& \mathrm{B}_{1}=\left[\begin{array}{lllll}
1 & 0 & 0 & \cdots & 0
\end{array}\right]^{\mathrm{T},} \\
& \mathrm{B}_{2}=\left[\begin{array}{lllll}
0 & 0 & 0 & \cdots & 1
\end{array}\right]^{\mathrm{T},}
\end{aligned}
$$




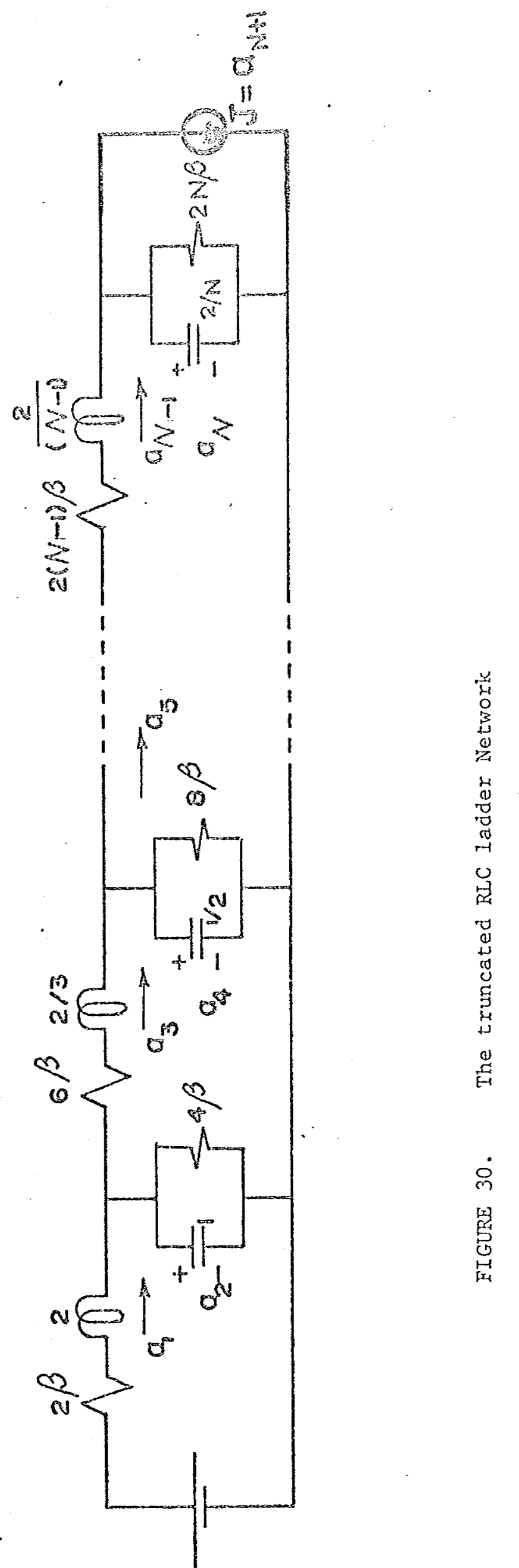




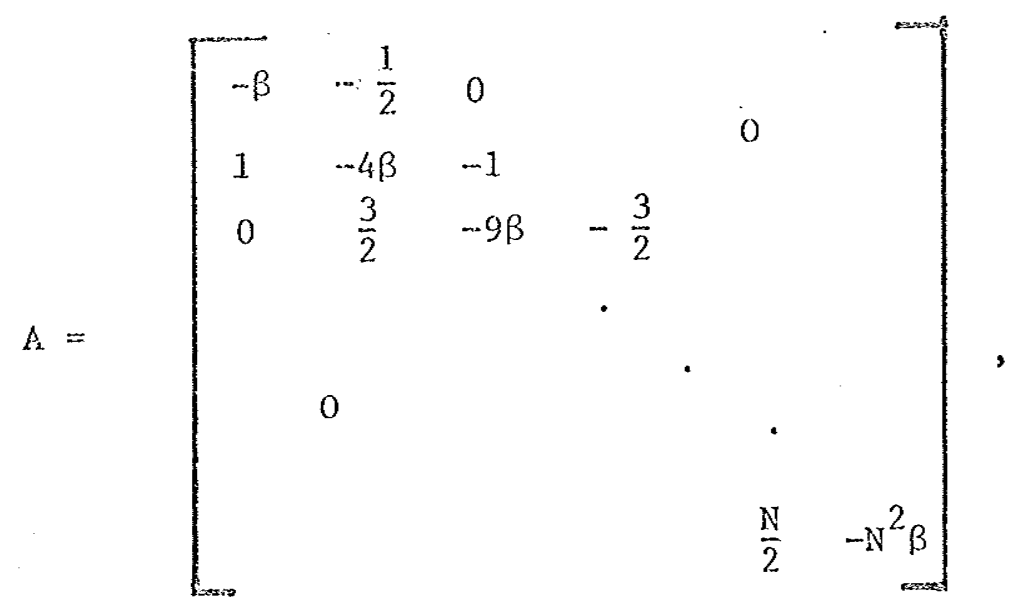

$$
\begin{aligned}
& \mathrm{U}_{1}=\frac{1}{2 \pi} \quad \text { and } \quad \mathrm{U}_{2}=-\frac{\mathrm{N}}{2} \mathrm{a}_{\mathrm{N}+1}
\end{aligned}
$$

Since the network is linear the responses $a_{n}(1 \leq n \leq N)$ are the sum of the individual responses due to $\mathrm{E}$, the initial conditions and $J$.

Let $\hat{a}$ be the response due to $E$ and the initial conditions and $\tilde{a}$ the response due to $\mathrm{J}$. Therefore,

$$
a=\hat{a}+\tilde{a}
$$

We now use a bound on $J$ to obtain a bound on $\|$ a $-\hat{a}||=\left.|||\tilde{a}|\right|_{2}$. Furthermore, by an appropriate choice of $N$ this bound can be made negligibly small. Thus $\hat{a}$ can be made a good approximation to the desire values a ; that is, $\hat{a}$ is obtained by solving the finite system by simply letting $\mathrm{J}=\mathrm{a}_{\mathrm{N}+1}=0$.

To obtain a bound on $a_{\mathrm{N}+1}$ we proceed as follow : The solutions of (3.13) for $\beta=0$, which corresponds to an LC ladder, are well behaved and the eigenvalues are all negative integers, $\left(\lambda_{n}=-n\right)$. Also the solutions of the special RLC ladder of Fig.9.b are well behaved and the eigenvalues ( see the second part of this Appendix) are all real and negative and to the left of the corresponding ones of the LC case, for every $n\left(\lambda_{n}=\cdots n\left(\beta^{2}+1\right)^{\frac{1}{2}}\right)$. On this basis one can reasonably assume 
that the RLC ladder of $\mathrm{Fig.9.a}$ is well. behaved and the eigenvalues of the system (3.13) will be to the left of the corresponding ones in the special RLC case since the damping is greater. Thus the response will be monotonic and also

$$
a_{n}(\tau) \leq C_{n}(\tau)
$$

Hence,

$$
J(\tau)=a_{N+1}(\tau) \leq C_{N+1}(\tau)
$$

To find the response due to $J$ we use the we11-known result for a finite linear system [22]:

$$
\tilde{a}=\int_{0}^{\tau} e^{A(\tau-t)} B_{2} a_{N+1}(t) d t
$$

By taking the Euclidean norm of both sides of (I.5) one obtains

$$
\|\tilde{a}\|_{2} \leq \int_{0}^{\tau}\left\|e^{A(\tau-t)}\right\|_{2} C_{N+1}(t) d t \quad
$$

Since the system is asymptotically stable, there exist positive $k_{1}$ and $k_{2}$ such that [42]

$$
\left\|e^{A(\tau-t)}\right\|_{2} \leq k_{2} e^{-k_{2}(\tau-t)}
$$

where $k_{l}$ can be taken as

$$
\mathrm{k}_{1}=\|\mathrm{P}\|_{2}\left\|\mathrm{P}^{-1}\right\|_{2}=\frac{\mu_{\max }}{\mu_{\min }}
$$

where $P$ is the modal matrix of $A$ and $\mu_{\max }$ and $\mu_{\min }$ axe the maximum and the minimum singular values of $P$ which are equal to the square root of the maximum and minimum eigenvalues of $\widetilde{\mathrm{P}}^{\mathrm{T}} \mathrm{P}$, respectively [43]. Thus

$$
\mathrm{k}_{1}=\left\{\frac{\max _{i}\left[\lambda_{1}\left(\overline{\mathrm{P}}^{\mathrm{T}} \mathrm{P}\right)\right]}{\min \left[\lambda_{i}\left(\overline{\mathrm{P}}^{\mathrm{T}} \mathrm{P}\right)\right]}\right\}^{\frac{1}{2}}
$$

where $\overrightarrow{\mathrm{P}}^{\mathrm{T}}$ is the complex conjugate transpose of $\mathrm{P}$. 
When the eigenvalues are distinct, as is the case here, $k_{2}$ is defined as the smallest (in magnitude) real part of the eigenvalues of $A$; that is,

$$
k_{2}=\underset{i}{\min } \mid \cdot \operatorname{Re} \lambda_{i}(A)
$$

A lower bound for $k_{2}$ has been given by Martens [44, equation (16)] in terms of the element values a network. For the network of Fig. 30 this bound is $\beta$. Thus,

$$
k_{2} \geq \beta
$$

Now for the case $a_{n}(0)=0, a_{N+1}(\tau)$ is bounded by the steady state value, since it is monotonic; that is,

$$
a_{N+1}(\tau) \leq a_{N+1}(\infty)=\frac{1}{\pi} \frac{I_{N+1}(1 / \beta)}{I_{0}(1 / \beta)}=M_{1}
$$

For the case when $a_{n}(0)=c_{n}(0)=\frac{1}{\pi}, a_{N+1}(\tau)$ is bounded by

$$
\begin{aligned}
& a_{N+1}(\tau) \leq C_{N+1}(\tau) \leq C_{N+1}\left(\tau_{0}\right)=M_{2}\left(\tau \geq \tau_{0} \geq 0\right) \quad \text { (I.13) } \\
& \text { Introducing }(I .9),(I .11),(I .12) \text { and }(I .13) \text { into (I.6) yields }
\end{aligned}
$$
after integrating,

$$
\|\tilde{a}\| \leq\left\{\frac{\max \left[\lambda_{i}\left(\overline{\mathrm{P}}^{\mathrm{T}} \mathrm{P}\right)\right]}{\min _{i}\left[\lambda_{i}\left(\overline{\mathrm{P}}^{\mathrm{T}} \mathrm{P}\right)\right]}\right\}^{\frac{1}{2}} \frac{\mathrm{M}_{1}}{\beta}
$$$$
\left(\text { for } a_{n}(0)=C_{n}(0)=0\right) \text { and }
$$

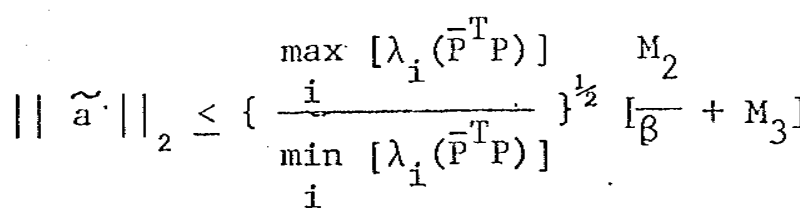

$\left(\right.$ for $\left.a_{n}(0)=c_{n}(0)=\frac{1}{\pi}\right)$, where.

$$
M_{3}=\int_{0}^{T_{0}} e^{-\beta\left(\tau_{0}-t\right)} C_{N+1}(t) d t .
$$


Nunerica1. Example

1- Consider the case of $a_{n}(0)=0, \beta=1.0$ and $N=20$

$\|\tilde{a}\|_{2} \leq 2.54 \times 10^{-27}$

2 - Consider the case of $a_{n}(0)=C_{n}(0)=\frac{1}{\pi}, \beta=1.0$ and $N=20$

\begin{tabular}{|c|c|}
\hline \multicolumn{1}{|c|}{$\tau_{0}$} & $\|\tilde{a}\|_{2} \leq$ \\
\hline 0.06 & $1.43 \times 10^{-3}$ \\
0.5 & $2.28 \times 10^{-5}$ \\
1.0 & $7.39 \times 10^{-8}$ \\
\hline
\end{tabular}

EVALUATION OF 'JHE EIGENVALJES

OF THE SYSTEM (3.38)

From (C.6) one can write

$$
c_{1}(\tau)=\frac{1}{\pi}\left\{b_{1} \tanh \left[\tau b_{1}+\operatorname{arctanh} b_{2}\right]-b_{1} b_{2}\right\}
$$

where $b_{1}=\left\{\beta^{2}+1\right\}^{\frac{1}{2}} \quad$ and $\quad b_{2}=\frac{\beta}{\left\{\beta^{2}+1\right\}^{\frac{1}{2}}}$

By letting $\theta=\operatorname{arctanh} b_{2}, C_{1}(\tau)$ can be written as

$$
c_{1}(\tau)=\frac{b_{1}}{\pi}\left\{\frac{1-e^{-\left(b_{1} \tau+2 \theta\right)}}{1+e^{-\left(b_{1} \tau+2 \theta\right)}}-b_{2}\right\}
$$




$$
\begin{aligned}
c_{1}(\tau) & =\frac{b_{1}}{\pi}\left\{-b_{2}+\left(1-e^{-\left(b_{1} \tau+2 \theta\right)}\right)\left(1-e^{-\left(b_{1} \tau+2 \theta\right)}+e^{-2\left(b_{1} \tau+2 \theta\right)}-\ldots\right.\right. \\
& \left.\left.(-1)^{n} e^{-n\left(b_{1} \tau+2 \theta\right)} \quad \ldots \ldots\right)\right\} \\
& =\frac{b_{1}}{\pi}\left(1-b_{2}\right)+\frac{2 b_{1}}{\pi} \sum_{n \geq 1}(-1)^{n} e^{-n b_{1} \tau} e^{-2 n \theta} \quad . \quad \text { (I. 16) }
\end{aligned}
$$

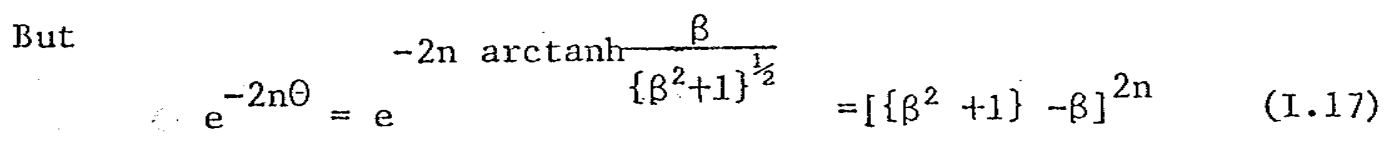

\section{Hence,}

$$
C_{1}(\tau)=\frac{1}{\pi}\left[\left\{\beta^{2}+1\right\}^{\frac{1}{2}}-\beta\right]+\frac{2}{\pi}\left\{\beta^{2}+1\right\}^{\frac{1}{2}} \sum_{n \geq 1}(-1)^{n}\left[\left\{\beta^{2}+1\right\}^{\frac{1}{2}}-\beta\right]^{2 n} e^{-n \tau\left\{\beta^{2}+1\right\}^{\frac{1}{2}}}
$$

But the general solution of the system can be written as

$$
c_{1}(\tau)=c_{1 s s}+\sum_{n \geq 1} d_{n 1} u_{n} e^{-\lambda_{n} \tau}
$$

where $C_{1 s s}$ is the steady state solution, $\mathrm{d}_{\mathrm{n} 1}$ are constant to be determined from the initial conditions, $\lambda_{\mathrm{n}}$ are the eigenvalues, and $U_{\mathbf{n}} \quad$ the corresponding eigenvalues.

Comparing (I.18) with (I.19) yields,

$$
\lambda_{n}=-n\left\{\beta^{2}+1\right\}^{\frac{1}{2}}
$$

